Supporting Information

\title{
A Chemoselective Layer-by-Layer Approach Utilizing Click Reactions with Ethynyl-cyclooctynes and Diazides
}

\author{
Niels Münster, Paul Nikodemiak, Ulrich Koert* \\ Fachbereich Chemie, Philipps-University Marburg \\ Hans-Meerwein-Strasse 4, D-35043 Marburg, Germany
}

*koert@chemie.uni-marburg.de

Experimental procedures, spectroscopic and analytical data for all new compounds 
General Methods and Materials

S1

Synthetic Procedures for the Preparation of Cyclooctyne 1

S2-S5

Alternative Route to alcohol 8

S6-S10

Synthetic Procedures for the Preparation of Cyclooctyne 2 S10-S14

Synthetic Procedures for the Preparation of diazide 3

S14-S16

Synthetic Procedures for Studies for the LbL appraoch

S16-S28

${ }^{1} \mathrm{H}-,{ }^{13} \mathrm{C}-\mathrm{NMR}$ spectra

S29-S58 


\section{General Methods and Materials}

All non-aqueous reactions were carried out using flame-dried glassware under argon atmosphere. All solvents were distilled by rotary evaporation. Solvents for non-aqueous reactions were dried as follows prior to use: THF and $\mathrm{Et}_{2} \mathrm{O}$ were dried with $\mathrm{KOH}$ and subsequently distilled from sodium/benzophenone (THF), respectively from Solvona ${ }^{\circledR}\left(\mathrm{Et}_{2} \mathrm{O}\right)$. $\mathrm{CH}_{2} \mathrm{Cl}_{2}$ was distilled from $\mathrm{CaH}_{2}$. $\mathrm{MeOH}$ was dried by refluxing with $\mathrm{Mg}$-turnings $(5 \mathrm{~g} / \mathrm{L})$ and subsequent distillation. All commercially available reagents and reactants were used without purification unless otherwise noted.

Reactions were monitored by thin layer chromatography (TLC) using Merck Silica Gel 60 F245plates and visualized by fluorescence quenching under UV-light. In addition, TLC-plates were stained using a cerium sulfate/phosphomolybdic acid stain. Chromatographic purification of products was performed on Merck Silica Gel 60 (230-400 mesh) unless otherwise noted using a forced flow of eluents. Concentration under reduced pressure was performed by rotary evaporation at $40{ }^{\circ} \mathrm{C}$ and appropriate pressure and by exposing to high vacuum at room temperature if necessary.

NMR spectra were recorded on a Bruker AV II 300 MHz, AV III HD 300 MHz, AV III 500 MHz, AV III HD $500 \mathrm{MHz}$ or AV II $600 \mathrm{MHz}$ spectrometer at room temperature. Chemical shifts are reported in ppm with the solvent resonance as internal standard. Data are reported as follows: $\mathrm{s}=$ singlet, $\mathrm{d}=$ doublet, $\mathrm{t}=$ triplet, $\mathrm{q}=$ quartet, quint $=$ quintet, $\mathrm{m}=$ multiplet.

Mass spectra were recorded by the mass service department of the Philipps-Universität Marburg. HR-ESI \& HR-APCI mass spectra were acquired with a LTQ-FT mass spectrometer (Thermo Fischer Scientific). The resolution was set to 100.000. HR-EI mass spectra were acquired with a MAT95 double focusing sector field mass spectrometer (Finnigan).

IR spectra were recorded on a Bruker IFS 200 spectrometer. The absorption bands are given in wave numbers $\left(\mathrm{cm}^{-1}\right)$, intensities are reported as follows: $\mathrm{s}=$ strong, $\mathrm{m}=$ medium, $\mathrm{w}=$ weak, br $=$ broad band.

Melting points were determined on a Stuart SMP10 or a Mettler Toledo MP70 using one end closed capillary tubes.

Elemental Analysis was performed on a $\mathrm{CHN}(\mathrm{S})$-Analysator vario MICRO CUBE. 


\section{Synthetic Procedures for the Preparation of Cyclooctyne 1}

tert-Butyl-1-methyl-5-oxocyclooctane-1-carboxylate (7)

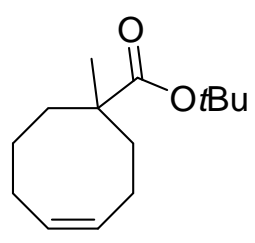

6
1) $\mathrm{ThBH}_{2}, \mathrm{H}_{2} \mathrm{O}_{2}, \mathrm{NaOH}, \mathrm{THF}, 0^{\circ} \mathrm{C}$

2) $\mathrm{PCC}, \mathrm{CH}_{2} \mathrm{Cl}_{2}$

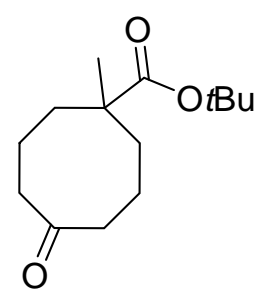

7

2,3-Dimethyl-2-butene (1.16 mL, $9.81 \mathrm{mmol}, 1.10 \mathrm{eq})$ was dissolved in THF $(40 \mathrm{~mL})$ at $0{ }^{\circ} \mathrm{C}$. $\mathrm{BH}_{3} \cdot \mathrm{Me}_{2} \mathrm{~S}(0.93 \mathrm{~mL}, 9.81 \mathrm{mmol}, 1.10 \mathrm{eq})$ was added and the resulting solution was stirred for $1 \mathrm{~h}$ at $0{ }^{\circ} \mathrm{C}$. Then a solution of Alkene $6^{[1]}(2.00 \mathrm{~g}, 8.92 \mathrm{mmol}, 1.00 \mathrm{eq})$ in THF $(10 \mathrm{~mL})$ was added dropwise. After stirring for $90 \mathrm{~min}$ at $0{ }^{\circ} \mathrm{C} 3 \mathrm{M}$ aq. $\mathrm{NaOH}(10 \mathrm{~mL})$ and $35 \mathrm{w} \%$ aq. $\mathrm{H}_{2} \mathrm{O}_{2}$ $(10 \mathrm{~mL})$ were added carefully. After $10 \mathrm{~min}$ the resulting mixture was warmed to $\mathrm{rt}$ and stirred at this temperature for $90 \mathrm{~min}$. Then sat. aq. $\mathrm{NaCl}(20 \mathrm{~mL})$ was added and the organic layer was separated. The aqueous layer was extracted with EtOAc $(3$ x $40 \mathrm{~mL})$. The combined organic layer was washed with sat. aq. $\mathrm{NaCl}(20 \mathrm{~mL})$ and dried over $\mathrm{MgSO}_{4}$. Evaporation of the solvents gave crude alcohol (mixture of Isomers) which was used for the next step without further purification.

The crude alcohol was dissolved in $\mathrm{CH}_{2} \mathrm{Cl}_{2}(45 \mathrm{~mL})$ and PCC (3.85 g, $\left.17.8 \mathrm{mmol}, 2.00 \mathrm{eq}\right)$ was added in portions at rt. The resulting mixture was stirred at $\mathrm{rt}$ for $1 \mathrm{~h}$. $\mathrm{Et}_{2} \mathrm{O}(100 \mathrm{~mL})$ was added and then the mixture was filtrated over a short plug of $\mathrm{Al}_{2} \mathrm{O}_{3}$ (neutral, activity $1,10 \mathrm{~g}$ ) which was washed with $\mathrm{Et}_{2} \mathrm{O}(100 \mathrm{~mL})$. The filtrate was concentrated under reduced pressure (r.r. 2:1 of the crude product). Column chromatography on silica (n-pentane/MTBE 7:1) gave pure ketone 7 (r.r $>25: 1,919 \mathrm{mg}, 3.82 \mathrm{mmol}, 43 \%$ over 2 steps) as a colorless oil. TLC (n-hexane/MTBE 5:1): $\mathrm{R}_{f}=0.23 .{ }^{1} \mathbf{H}$ NMR (500 MHz, $\left.\mathrm{CDCl}_{3}\right): \delta=1.10\left(\mathrm{~s}, 3 \mathrm{H}, \mathrm{CH}_{3}\right), 1.44(\mathrm{~s}$, $9 \mathrm{H}, \mathrm{C}\left(\mathrm{CH}_{3}\right)_{3}, 1.50$ (ddd, $\left.J=15.2,9.3,2.5 \mathrm{~Hz}, 2 \mathrm{H}, 2-\mathrm{H}_{a}, 8-\mathrm{H}_{a}\right), 1.69-1.87$ (m, 4H, 3-H, 7-H), 2.01 (ddd, $J=15.2,8.7,2.5 \mathrm{~Hz}, 2 \mathrm{H}, 2-\mathrm{H}_{b}, 8-\mathrm{H}_{b}$ ), 2.38 (ddd, $J=12.6,7.6,5.1 \mathrm{~Hz}, 2 \mathrm{H}, 4-\mathrm{H}_{a}, 6-$ $\left.\mathrm{H}_{a}\right), 2.51$ (ddd, $\left.J=12.6,8.6,5.2 \mathrm{~Hz}, 2 \mathrm{H}, 4-\mathrm{H}_{b}, 6-\mathrm{H}_{b}\right) .{ }^{13} \mathrm{C}$ NMR $\left(125 \mathrm{MHz}, \mathrm{CDCl}_{3}\right): \delta=20.8$ $(2 \mathrm{C}, \mathrm{C} 3+\mathrm{C} 7), 26.3\left(\mathrm{CH}_{3}\right), 28.2\left(\mathrm{C}\left(\mathrm{CH}_{3}\right)_{3}\right), 34.1(2 \mathrm{C}, \mathrm{C} 2+\mathrm{C} 8), 43.8(2 \mathrm{C}, \mathrm{C} 4+\mathrm{C} 6), 45.7(\mathrm{C} 1)$, $80.3\left(\mathrm{C}\left(\mathrm{CH}_{3}\right)_{3}\right), 176.9(\mathrm{COO} t \mathrm{Bu}), 216.3(\mathrm{C} 5)$. HR-MS $(\mathrm{EI}): \mathrm{m} / \mathrm{z}$ calcd for $\mathrm{C}_{14} \mathrm{H}_{24} \mathrm{O}_{3}[\mathrm{M}]^{+}$: 240.1726; found: 240.1733. FT-IR (neat): $\widetilde{v}=2972$ (w), 2935 (w), 1716 (s), 1703 (s), 1475 (w), 1392 (m), 1324 (w), 1252 (m), 1158 (s), 1123 (s), 849 (w).

${ }^{[1]}$ H. A. Kostalik IV, T. J. Clark, N. J. Robertson, P. F. Mutolo, J. M. Longo, H. D. Abruna, G. W. Coates, Macromolecules 2010, 43, 7147-7150. 
(E)-5-(Hydroxymethyl)-5-methylcyclooct-1-en-1-yl trifluoromethanesulfonate (8)

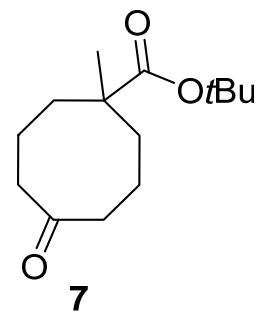

\author{
1) $\mathrm{KHMDS}, \mathrm{PhN}(\mathrm{Tf})_{2}, \mathrm{THF},-78^{\circ} \mathrm{C}$ \\ 2) DIBAH, $\mathrm{CH}_{2} \mathrm{Cl}_{2} / \mathrm{THF},-20^{\circ} \mathrm{C}$ to rt
}

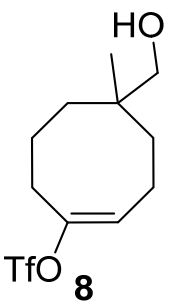

Ketone 7 (3.68 g, $15.3 \mathrm{mmol}, 1.00 \mathrm{eq})$ and $\mathrm{PhN}(\mathrm{Tf})_{2}(5.74 \mathrm{~g}, 16.1 \mathrm{mmol}, 1.05 \mathrm{eq})$ were dissolved in THF $(70 \mathrm{~mL})$ and cooled to $-78{ }^{\circ} \mathrm{C}$. KHMDS $(0.5 \mathrm{M}$ in toluene, $33.7 \mathrm{~mL}$, $16.8 \mathrm{mmol}, 1.10 \mathrm{eq}$ ) was added over a period of $15 \mathrm{~min}$. The resulting mixture was stirred for additionally $20 \mathrm{~min}$ at the same temperature. Then $\mathrm{H}_{2} \mathrm{O}(50 \mathrm{~mL})$ was added and the solution was warmed to rt. MTBE $(150 \mathrm{~mL})$ was added and the organic layer was separated. The organic layer was washed with sat. aq. $\mathrm{Na}_{2} \mathrm{CO}_{3}(2 \times 50 \mathrm{~mL})$ and sat. aq. $\mathrm{NaCl}(50 \mathrm{~mL})$ and then dried over $\mathrm{MgSO}_{4}$. The solvents were removed under reduced pressure to give the triflate $(5.93 \mathrm{~g}$, $15.9 \mathrm{mmol}, 104 \%$ ) which contains minor amounts of $\mathrm{PhN}(\mathrm{Tf})_{2}$. An analytically pure sample was obtained by chromatography on silica (n-pentane/Et ${ }_{2} \mathrm{O} 20: 1$ ) of a small amount of the compound. TLC ( $n$-hexane/MTBE 40:1): $\mathrm{R}_{f}=0.10 .{ }^{1} \mathbf{H}$ NMR (400 MHz, $\mathrm{C}_{6} \mathrm{D}_{6}$ ): $\delta=0.94$ (s, $\left.3 \mathrm{H}, \mathrm{CH}_{3}\right), 1.07$ (ddd, $\left.J=14.9,9.6,2.2 \mathrm{~Hz}, 1 \mathrm{H}, 2-\mathrm{H}_{a}\right), 1.30\left(\mathrm{~s}, 9 \mathrm{H}, \mathrm{C}\left(\mathrm{CH}_{3}\right) 3\right), 1.32-1.40(\mathrm{~m}, 1 \mathrm{H}$, 8- $\left.\mathrm{H}_{a}\right), 1.42-1.51\left(\mathrm{~m}, 1 \mathrm{H}, 7-\mathrm{H}_{a}\right), 1.55-1.72\left(\mathrm{~m}, 3 \mathrm{H}, 3-\mathrm{H}_{b}, 7-\mathrm{H}_{b}, 8-\mathrm{H}_{a}\right), 1.93-2.03\left(\mathrm{~m}, 1 \mathrm{H}, 8-\mathrm{H}_{b}\right)$, 2.04-2.13 (m, 2H, 2-Hb, 6-Ha), 2.41 (ddd, $J=15.9,9.5,6.1 \mathrm{~Hz}, 1 \mathrm{H}, 6-\mathrm{H} b), 5.47$ (t, $J=6.5 \mathrm{~Hz}$,

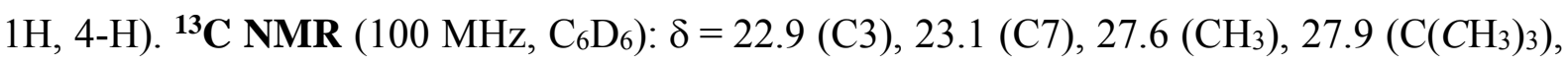
29.5 (C6), 32.9 (C8), 34.8 (C2), $46.1(\mathrm{C} 1), 79.7\left(C\left(\mathrm{CH}_{3}\right)_{3}\right), 119.3$ (q, J= 320 Hz, $\left.\mathrm{CF}_{3}\right), 123.2$ (C4), 148.7 (C5), 175.8 (COOtBu). ${ }^{19}$ F NMR (282 MHz, $\left.\mathrm{C}_{6} \mathrm{D}_{6}\right): \delta=-75.4$. HR-MS (APCI): $\mathrm{m} / \mathrm{z}$ calcd for $\mathrm{C}_{15} \mathrm{H}_{23} \mathrm{~F}_{3} \mathrm{O}_{5} \mathrm{SNa}[\mathrm{M}+\mathrm{Na}]^{+}:$395.1111; found: 395.1103. FT-IR (neat): $\tilde{v}=2976(\mathrm{w}), 2936(\mathrm{w}), 1717(\mathrm{~m}), 1413(\mathrm{~m}), 1368(\mathrm{w}), 1247(\mathrm{~m}), 1203(\mathrm{~s}), 1142(\mathrm{~s}), 1032(\mathrm{w})$, $930(\mathrm{~m}), 843(\mathrm{~s}), 612(\mathrm{~m}), 516(\mathrm{~m})$.

The vinyl triflate $(5.41 \mathrm{~g}, 14.5 \mathrm{mmol}, 1.00 \mathrm{eq})$ was dissolved in $\mathrm{CH}_{2} \mathrm{Cl}_{2} / \mathrm{THF}$ (7:1, $\left.49 \mathrm{~mL}\right)$ and cooled to $-15{ }^{\circ} \mathrm{C}$. DIBAH (1.2 $\mathrm{M}$ in toluene, $\left.30.3 \mathrm{~mL}, 36.3 \mathrm{mmol}, 2.50 \mathrm{eq}\right)$ was added. After stirring for $15 \mathrm{~min}$ at this temperature the cooling bath was removed and the reaction mixture was stirred at $\mathrm{rt}$ for $16 \mathrm{~h}$. A sat. aq. solution of sodium potassium tartrate $(40 \mathrm{~mL})$ and $\mathrm{CH}_{2} \mathrm{Cl}_{2}$ $(40 \mathrm{~mL})$ were added and the resulting mixture was stirred for $1 \mathrm{~h}$. The organic layer was separated and the aqueous layer was extracted with $\mathrm{CH}_{2} \mathrm{Cl}_{2}(3 \times 40 \mathrm{~mL})$. The combined organic layer was dried over $\mathrm{MgSO}_{4}$ and the solvents were removed under reduced pressure. Purification by column chromatography on silica (n-pentane/MTBE 2:1) gave the alcohol 8 (3.03 g, $10.0 \mathrm{mmol}, 69 \%$ ) as a colorless liquid. TLC (n-hexane/MTBE 2:1): $\mathrm{R}_{f}=0.16 .{ }^{1} \mathbf{H}$ NMR 
$\left(500 \mathrm{MHz}\right.$, acetone- $\left.d_{6}\right): \delta=0.93\left(\mathrm{~s}, 3 \mathrm{H}, \mathrm{CH}_{3}\right), 1.42\left(\mathrm{ddd}, J=14.9,7.2,4.2 \mathrm{~Hz}, 1 \mathrm{H}, 6-\mathrm{H}_{a}\right), 1.48-$ $56\left(\mathrm{~m}, 2 \mathrm{H}, 6-\mathrm{H}_{b}, 4-\mathrm{H}_{a}\right), 1.71-1.86\left(\mathrm{~m}, 3 \mathrm{H}, 4-\mathrm{H}_{b}, 7-\mathrm{H}\right), 2.27-2.33(\mathrm{~m}, 2 \mathrm{H}, 3-\mathrm{H}), 2.58$ (ddd, $J=$ $\left.16.2,6.6,6.6 \mathrm{~Hz}, 1 \mathrm{H}, 8-\mathrm{H}_{a}\right), 2.71\left(\mathrm{ddd}, J=15.8,8.0,7.7 \mathrm{~Hz}, 1 \mathrm{H}, 8-\mathrm{H}_{b}\right), 3.28\left(\mathrm{~s}, 2 \mathrm{H}, \mathrm{CH}_{2} \mathrm{OH}\right)$, 3.68 (bs, 1H, OH), 5.95 (t, $J=6.0 \mathrm{~Hz}, 1 \mathrm{H}, 2-\mathrm{H}) .{ }^{13} \mathrm{C}$ NMR $\left(125 \mathrm{MHz}\right.$, acetone- $\left.d_{6}\right): \delta=22.8$ (C7), 23.2 (C3), 23.5 ( $\left.\mathrm{CH}_{3}\right), 30.32$ (C8), 31.4 (C6), 34.1 (C4), 39.3 (C5), $71.5\left(\mathrm{CH}_{2} \mathrm{OH}\right), 119.5$ (q, $\left.J=319 \mathrm{~Hz}, \mathrm{CF}_{3}\right), 124.6(\mathrm{C} 2), 149.3(\mathrm{C} 1) .{ }^{19} \mathbf{F}$ NMR (282 MHz, acetone-d6): $\delta=-74.0$. Elem. Anal. calcd. for $\mathrm{C}_{11} \mathrm{H}_{17} \mathrm{~F}_{3} \mathrm{O}_{4} \mathrm{~S}: \mathrm{C}, 43.70 ; \mathrm{H}, 5.67 ; \mathrm{N}, 0.00 ; \mathrm{S}, 10.61$. Found: $\mathrm{C}, 43.54 ; \mathrm{H}$, 5.73; N, 0.01; S, 10.30. FT-IR (neat): $\widetilde{v}=3351$ (w), 2938 (w), $2872(\mathrm{w}), 1686(\mathrm{w}), 1409$ (s), 1200 (s), 1138 (s), 1023 (m), 982 (m), 934 (m), 821 (m), 766 (w), 607 (s), 510 (m).

\section{(E)-5-Ethynyl-5-methylcyclooct-1-en-1-yl trifluoromethanesulfonate (9)}

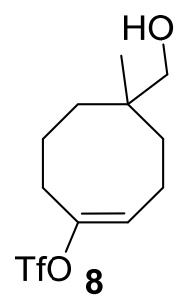

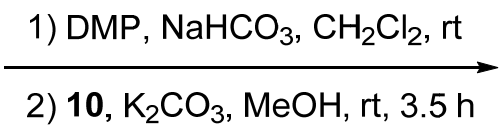
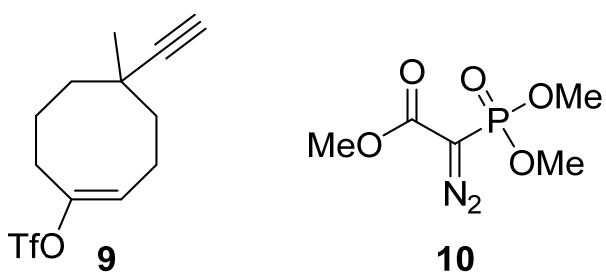

Alcohol 8 (1.00 g, $3.31 \mathrm{mmol}, 1.00 \mathrm{eq})$ was dissolved in $\mathrm{CH}_{2} \mathrm{Cl}_{2}(20 \mathrm{~mL})$ and cooled to $0{ }^{\circ} \mathrm{C}$. $\mathrm{NaHCO}_{3}$ (834 mg, $9.92 \mathrm{mmol}, 3.00 \mathrm{eq}$ ) and DMP (1.54 g, $3.64 \mathrm{mmol}, 1.10 \mathrm{eq}$ ) were added and the cooling bath was removed. The resulting mixture was stirred at rt for $30 \mathrm{~min}$ and then diluted with $n$-pentane $(25 \mathrm{~mL})$. It was filtrated over a short plug of celite which was washed with $\mathrm{Et}_{2} \mathrm{O}$ $(50 \mathrm{~mL})$. The filtrate was concentrated to give the crude aldehyde which was used in the next step without further purification.

The crude aldehyde was dissolved in $\mathrm{MeOH}(20 \mathrm{~mL})$ and $\mathrm{K}_{2} \mathrm{CO}_{3}(1.37 \mathrm{~g}, 9.92 \mathrm{mmol}, 3.00 \mathrm{eq})$ was added. The mixture was stirred at $\mathrm{rt}$ for $15 \mathrm{~min}$, then Ohira-Bestmann-reagent (10) (762 mg, $3.97 \mathrm{mmol}, 1.20 \mathrm{eq}$ ) was added. The reaction mixture was stirred for $3.5 \mathrm{~h}$ at $\mathrm{rt}$ and then it was poured into a mixture of $\mathrm{Et}_{2} \mathrm{O}(50 \mathrm{~mL})$ and sat. aq. $\mathrm{NaHCO}_{3}(20 \mathrm{~mL})$. The organic layer was separated and aqueous layer was extracted with $\mathrm{Et}_{2} \mathrm{O}(1 \times 50 \mathrm{~mL})$. The combined organic layer was washed with sat. aq. $\mathrm{NaCl}(20 \mathrm{~mL})$ and dried over $\mathrm{MgSO}_{4}$. The solvents were removed under reduced pressure. The remaining crude product was purified by flash chromatography on silica (n-pentane/Et $2 \mathrm{O} 20: 1)$ to give alkyne 9 (728 mg, $2.46 \mathrm{mmol}, 74 \%$ over 2 steps) as a colorless oil. TLC ( $n$-hexane/MTBE 20:1): $\mathrm{R}_{f}=0.58 .{ }^{1} \mathbf{H}$ NMR $(500 \mathrm{MHz}$, $\left.\mathrm{C}_{6} \mathrm{D}_{6}\right): \delta=0.91-0.98\left(\mathrm{~m}, 1 \mathrm{H}, 4-\mathrm{H}_{a}\right), 0.98\left(\mathrm{~s}, 3 \mathrm{H}, \mathrm{CH}_{3}\right), 1.25-1.34(\mathrm{~m}, 2 \mathrm{H}, 6-\mathrm{H}), 1.42-1.57(\mathrm{~m}$, 3H, 3-Ha, 4-Hb, 7-Ha), 1.69-1.77 (m, 1H, 7-Hb), 1.87 (s, 1H, CHAlkyne), 1.99-2.06 (m, 2H, 3-Hb, 8-Ha), $2.65\left(\mathrm{ddd}, J=15.8,11.2,6.2 \mathrm{~Hz}, 1 \mathrm{H}, 8-\mathrm{H}_{b}\right), 5.42(\mathrm{t}, J=7.8 \mathrm{~Hz}, 1 \mathrm{H}, 2-\mathrm{H}) .{ }^{13} \mathbf{C}$ NMR 
(125 MHz, $\left.\mathrm{C}_{6} \mathrm{D}_{6}\right): \delta=23.1(\mathrm{C} 3), 23.8(\mathrm{C} 7), 28.6(\mathrm{C} 8), 32.7\left(\mathrm{CH}_{3}\right), 35.2$ (C6), 35.3 (C5), 41.6 (C4), $71.4(\mathrm{CCH}), 88.7(\mathrm{CCH}), 119.2$ (q, $\left.J=320 \mathrm{~Hz}, \mathrm{CF}_{3}\right), 122.1(\mathrm{C} 2), 149.8(\mathrm{C} 1) .{ }^{\mathbf{1 9}} \mathbf{F}$ NMR (282 MHz, $\left.\mathrm{C}_{6} \mathrm{D}_{6}\right): \delta=-74.0$. HR-MS (EI): $\mathrm{m} / \mathrm{z}$ calcd for $\mathrm{C}_{12} \mathrm{H}_{15} \mathrm{O}_{3} \mathrm{~F}_{3} \mathrm{~S}[\mathrm{M}]^{+}:$296.0694; found: 296.0675. FT-IR (neat): $\widetilde{v}=3303(\mathrm{w}), 2970(\mathrm{w}), 2933(\mathrm{w}), 1462(\mathrm{w}), 1414(\mathrm{~m}), 1206(\mathrm{~s}), 1141$ (s), $1038(\mathrm{w}), 941(\mathrm{~m}), 869(\mathrm{~m}), 827(\mathrm{w}), 636(\mathrm{~m}), 612(\mathrm{~m}), 524(\mathrm{w})$.

\section{5-Ethynyl-5-methylcyclooct-1-yne (1)}

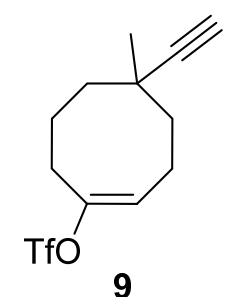

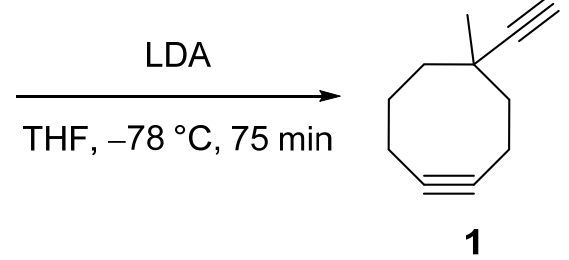

Alkyne 9 (200 mg, $0.66 \mathrm{mmol}, 1.00 \mathrm{eq})$ was dissolved in THF $(3.0 \mathrm{~mL})$ and cooled to $-78{ }^{\circ} \mathrm{C}$. Freshly prepared LDA (1.0 M in THF/n-hexane, $4.8 \mathrm{~mL}, 4.76 \mathrm{mmol}, 3.00 \mathrm{eq})$ was added dropwise and the resulting solution was stirred at this temperature for $75 \mathrm{~min}$. The reaction was quenched by addition of $\mathrm{H}_{2} \mathrm{O}(10 \mathrm{~mL})$ and warmed to $\mathrm{rt}$. The mixture was extracted with $\mathrm{Et}_{2} \mathrm{O}$ $(2 \times 30 \mathrm{~mL})$. The combined organic layer was washed with sat. aq. $\mathrm{NaCl}(20 \mathrm{~mL})$ and dried over $\mathrm{MgSO}_{4}$. The solvents were removed under reduced pressure and the remaining crude product was purified by column chromatography on silica (n-pentane/Et $\mathrm{t}_{2} \mathrm{O} 50: 1$ ) to afford cyclooctyne 1 (155 mg, $1.06 \mathrm{mmol}, 67 \%$ ) as a colorless liquid. TLC (n-hexane/MTBE 100:1): $\mathrm{R}_{f}=0.33 .{ }^{1} \mathbf{H}$ NMR $\left(500 \mathrm{MHz}, \mathrm{C}_{6} \mathrm{D}_{6}\right): \delta=1.17\left(\mathrm{~s}, 3 \mathrm{H}, \mathrm{CH}_{3}\right), 1.46-1.52\left(\mathrm{~m}, 1 \mathrm{H}, 6-\mathrm{H}_{\mathrm{a}}\right), 1.61-$ $1.68\left(\mathrm{~m}, 3 \mathrm{H}, 4-\mathrm{H}_{a}, 6-\mathrm{H}_{b}, 8-\mathrm{H}_{a}\right), 1.72-1.77\left(\mathrm{~m}, 1 \mathrm{H}, 4-\mathrm{H}_{b}\right), 1.83-1.90\left(\mathrm{~m}, 1 \mathrm{H}, 3-\mathrm{H}_{a}\right), 1.91-1.96$ (m, 1H, 7-H), 2.09 (s, 1H, CCH), 2.24-2.34 (m, 1H, 8- $\left.\mathrm{H}_{b}\right), 2.48-2.56\left(\mathrm{~m}, 1 \mathrm{H}, 3-\mathrm{H}_{b}\right) .{ }^{13} \mathbf{C}$ NMR (125 MHz, $\left.\mathrm{C}_{6} \mathrm{D}_{6}\right): \delta=18.4(\mathrm{C} 3), 21.3(\mathrm{C} 7), 29.8$ (C8), $34.2\left(\mathrm{CH}_{3}\right), 35.9$ (C5), 41.3 (C6), 47.1 (C4), $72.3(\mathrm{CCH}), 88.8(\mathrm{CCH}), 95.4(\mathrm{C} 2), 96.4(\mathrm{C} 1)$. HR-MS (EI): $\mathrm{m} / \mathrm{z}$ calcd for $\mathrm{C}_{11} \mathrm{H}_{14}[\mathrm{M}]^{+}$: 146.1095; found: 146.1084. FT-IR (neat): $\widetilde{v}=3293$ (m), 2966 (m), 2928 (m), 2107 (w), 1705 (m), 1441 (m), 1315 (w), 1177 (m), 1142 (m), 1039 (w), 941 (w), 869 (m), 625 (s), 532 (m), $431(\mathrm{~m})$. 


\section{Alternative Route to Alcohol 8}<smiles>CCCCC1(C)CCC=CCCC1</smiles>

6

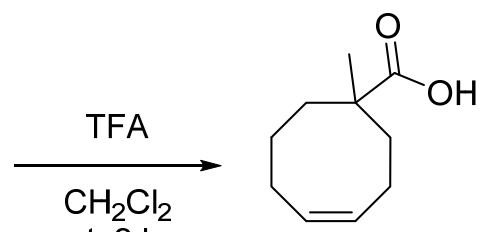

S1 quant.
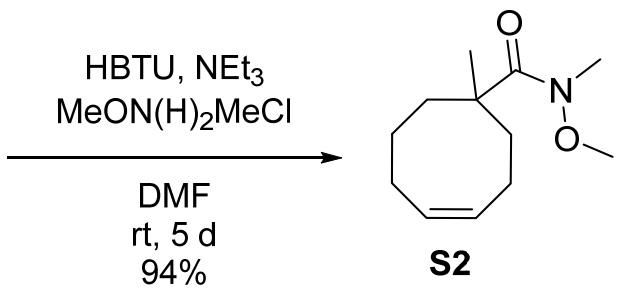

$94 \%$

$\mathrm{s} 2$

1. $\mathrm{ThBH}_{2}$;

$\mathrm{H}_{2} \mathrm{O}_{2}, \mathrm{NaOH}, \mathrm{THF}, 0^{\circ} \mathrm{C}$ 2. TPAP, NMO, $4 \AA \mathrm{MS}$ $\mathrm{CH}_{2} \mathrm{Cl}_{2}, \mathrm{rt}, 16 \mathrm{~h}$
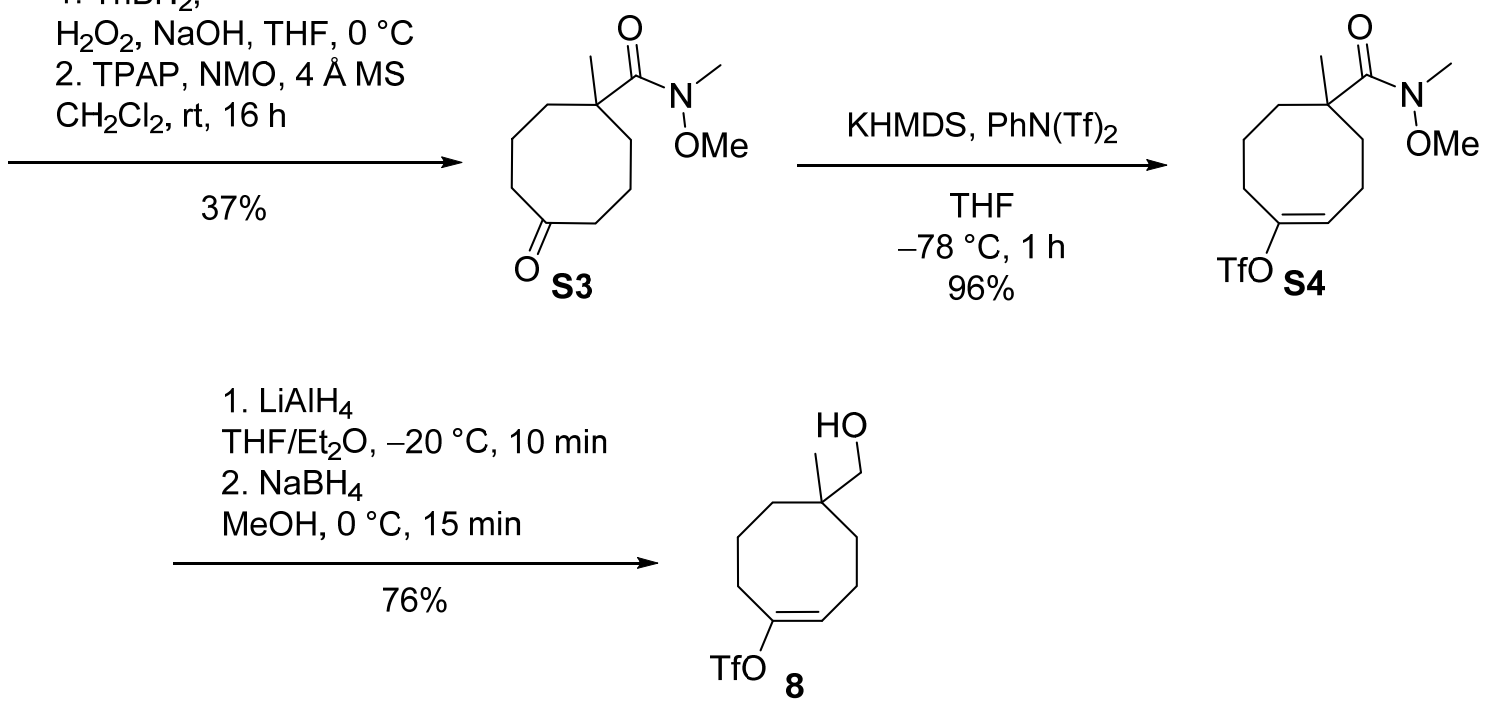

(Z)-1-Methylcyclooct-4-ene-1-carboxylic acid (S1)<smiles>CCCCOC(=O)C1(C)CCCCCCC1</smiles>

6

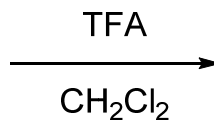

rt, $3 \mathrm{~h}$<smiles>CC1(C(=O)O)CCC=CCCC1</smiles>

S1

Ester 6 (30.0 g, $134 \mathrm{mmol}, 1.00 \mathrm{eq})$ was dissolved in $\mathrm{CH}_{2} \mathrm{Cl}_{2}(40 \mathrm{~mL})$ and TFA (51.5 mL, $669 \mathrm{mmol}, 5.00 \mathrm{eq}$ ) was added. After stirring for $3 \mathrm{~h}$ at rt, the solution was cooled to $0{ }^{\circ} \mathrm{C}$ and $3 \mathrm{M}$ aq. $\mathrm{NaOH}(50 \mathrm{~mL})$ was added slowly. Sat. aq. $\mathrm{NaHCO}_{3}$ was added until a $\mathrm{pH}$ of 4 was reached. The organic layer was separated and the aqueous layer was extracted with $\mathrm{CH}_{2} \mathrm{Cl}_{2}(4 \mathrm{x}$ $50 \mathrm{~mL})$. The combined organic layer was washed with sat. aq. $\mathrm{NaCl}(50 \mathrm{~mL})$ and dried over $\mathrm{MgSO}_{4}$. The solvent was removed under reduced pressure to give acid S1 (22.5 g, $134 \mathrm{mmol}$, quant.) as a colorless solid. m.p. $\left(\mathrm{CH}_{2} \mathrm{Cl}_{2}\right): 52{ }^{\circ} \mathrm{C}$. TLC (n-hexane/EtOAc 2:1): $\mathrm{R}_{f}=0.47 .{ }^{1} \mathbf{H}$ NMR $\left(500 \mathrm{MHz}, \mathrm{CDCl}_{3}\right): \delta=1.25\left(\mathrm{~s}, 3 \mathrm{H}, \mathrm{CH}_{3}\right), 1.57-1.72\left(\mathrm{~m}, 4 \mathrm{H}, 2-\mathrm{H}_{a}, 3-\mathrm{H}_{a}, 7-\mathrm{H}_{a}, 8-\mathrm{H}_{a}\right)$, 1.84-1.89 (m, 1H, 2-Hb), 2.11-2.38 (m, 5H, 3- $\left.\mathrm{H}_{b}, 6-\mathrm{H}, 7-\mathrm{H}_{b}, 8-\mathrm{H}_{b}\right), 5.43-5.52$ (m, 1H, Holefin), 5.65-5.72 (m, 1H, Holefin). ${ }^{13} \mathrm{C}$ NMR (125 MHz, $\mathrm{CDCl}_{3}$ ): $\delta=24.6$ (C7), 24.7 (C3), 25.9 (C6), $27.0\left(\mathrm{CH}_{3}\right), 32.2(\mathrm{C} 2), 35.3(\mathrm{C} 8), 46.2(\mathrm{C} 1), 126.6$ (Colefin), 131.9 (Colefin), 184.1 (COOH). HRMS (EI): $m / z$ calcd for $\mathrm{C}_{10} \mathrm{H}_{16} \mathrm{O}_{2}[\mathrm{M}]^{+}: 168.1150$; found: 168.1155 . FT-IR (neat): $\widetilde{v}=2933$ 
(m), 2875 (m), 1686 (s), 1478 (m), 1404 (m), 1373 (w), 1211 (s), 1119 (m), 1024 (w), 931 (m), 816 (w), $744(\mathrm{~m}), 660(\mathrm{~m}), 533(\mathrm{~m}), 398(\mathrm{w})$.

\section{(Z)- $N$-methoxy- $N$,1-dimethylcyclooct-4-ene-1-carboxamide (S2)}

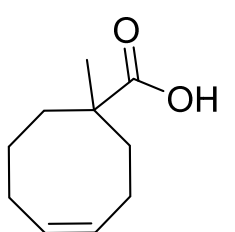

S1

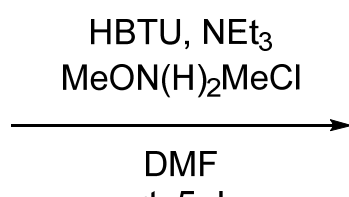

rt, $5 \mathrm{~d}$

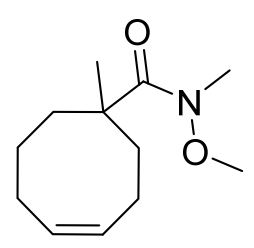

S2

To a solution of acid S1 (20.0 g, $119 \mathrm{mmol}, 1.00 \mathrm{eq})$ in DMF (150 mL) were added Weinreb's salt (18.3 g, $187 \mathrm{mmol}, 1.50 \mathrm{eq}), \mathrm{NEt}_{3}(66 \mathrm{~mL}, 476 \mathrm{mmol}, 4.00 \mathrm{eq})$ and HBTU (47.3 g, 125 mmol, $1.05 \mathrm{eq}$ ) and the reaction mixture was stirred for $5 \mathrm{~d}$ at $\mathrm{rt}$. The reaction was then poured into a mixture of MTBE $(400 \mathrm{~mL})$ and sat. aq. $\mathrm{NH}_{4} \mathrm{Cl}(400 \mathrm{~mL})$. The organic layer was separated and the aqueous layer was extracted with MTBE $(2 \times 200 \mathrm{~mL})$. The combined organic layer was dried over $\mathrm{MgSO}_{4}$ and the solvents were removed under reduced pressure. The remaining crude product was purified by column chromatography on silica ( $n$-pentane/MTBE 5:1) to give Weinreb amide S2 $(23.6 \mathrm{~g}, 111 \mathrm{mmol}, 94 \%)$ as a colorless oil. TLC ( $n$ hexane/EtOAc 4:1): $\mathrm{R}_{f}=0.28 .{ }^{1} \mathbf{H}$ NMR $\left(400 \mathrm{MHz}, \mathrm{CDCl}_{3}\right): \delta=1.24\left(\mathrm{~s}, 3 \mathrm{H}, \mathrm{CH}_{3}\right), 1.39$ (ddd, $\left.J=14.5,9.2,2.0 \mathrm{~Hz}, 1 \mathrm{H}, 2-\mathrm{H}_{a}\right), 1.47-1.72\left(\mathrm{~m}, 3 \mathrm{H}, 3-\mathrm{H}, 8-\mathrm{H}_{a}\right), 1.96-2.06\left(\mathrm{~m}, 2 \mathrm{H}, 7-\mathrm{H}_{a}, 8-\mathrm{H}_{a}\right)$, 2.08-2.19 (m, 1H, 6- $\left.\mathrm{H}_{a}\right), 2.29-2.56\left(\mathrm{~m}, 3 \mathrm{H}, 2-\mathrm{H}_{b}, 6-\mathrm{H}_{b}, 7-\mathrm{H}_{b}\right), 3.20\left(\mathrm{~s}, 3 \mathrm{H}, \mathrm{NCH}_{3}\right), 3.68(\mathrm{~s}, 3 \mathrm{H}$, $\left.\mathrm{OCH}_{3}\right), 5.42-5.51(\mathrm{~m}, 1 \mathrm{H}, 5-\mathrm{H}), 5.68-5.76(\mathrm{~m}, 1 \mathrm{H}, 4-\mathrm{H}) .{ }^{13} \mathbf{C} \quad \mathbf{N M R}(100 \mathrm{MHz}$, $\left.\mathrm{CDCl}_{3}\right): \delta=24.7(\mathrm{C} 7), 25.5(\mathrm{C} 3), 25.5(\mathrm{C} 6), 25.7\left(\mathrm{CH}_{3}\right), 31.5(\mathrm{C} 8), 34.1\left(\mathrm{NCH}_{3}\right), 36.6,(\mathrm{C} 2)$, 47.0 (C1), $60.4\left(\mathrm{OCH}_{3}\right), 128.8(\mathrm{C} 5), 132.5$ (C4), $179.2\left(\mathrm{CONCH}_{3} \mathrm{OCH}_{3}\right) . \mathbf{H R}-\mathrm{MS}(\mathrm{ESI}): \mathrm{m} / \mathrm{z}$ calcd for $\mathrm{C}_{12} \mathrm{H}_{2} \mathrm{NO}_{2} \mathrm{Na}[\mathrm{M}+\mathrm{Na}]^{+}: 234.1465$; found: 234.1468. FT-IR (neat): $\widetilde{v}=3005(\mathrm{~m})$, 2929 (m), 1724 (w), 1641 (s), 1452 (m), 1337 (m), 1224 (m), 1110 (m), 993 (s), 843 (w), $734(\mathrm{~m}), 660(\mathrm{~m}), 549(\mathrm{~m}), 428(\mathrm{~m})$.

\section{$N$-Methoxy- $N$,1-dimethyl-5-oxocyclooctane-1-carboxamide (S3)}<smiles>CON(C)C(=O)C1(C)CCC=CCCC1</smiles>

S2

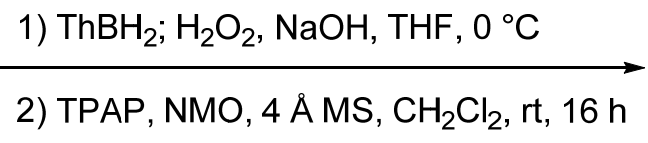

2) TPAP, NMO, $4 \AA \mathrm{MS}, \mathrm{CH}_{2} \mathrm{Cl}_{2}$, rt, $16 \mathrm{~h}$

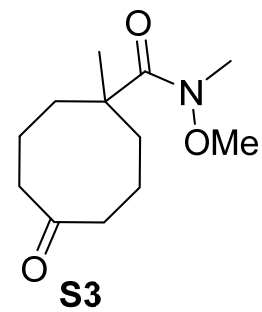

The thexylborane solution was prepared by addition of $\mathrm{BH}_{3} \cdot \mathrm{Me}_{2} \mathrm{~S}(1.24 \mathrm{~mL}, 13.0 \mathrm{mmol}$, $1.10 \mathrm{eq})$ to a solution of 2,3-dimethyl-2-butene (1.29 g, $15.4 \mathrm{mmol}, 1.30 \mathrm{eq})$ in THF (40 mL) at $0{ }^{\circ} \mathrm{C}$. After stirring for $1 \mathrm{~h}$ a solution of alkene S2 $(2.50 \mathrm{~g}, 11.8 \mathrm{mmol}, 1.00 \mathrm{eq})$ in THF 
$(10 \mathrm{~mL})$ was added and the resulting mixture was stirred at $0{ }^{\circ} \mathrm{C}$ for $20 \mathrm{~min}$. Then aq. $3 \mathrm{M} \mathrm{NaOH}$ $(8 \mathrm{~mL})$ and aq. $35 \mathrm{w} \% \mathrm{H}_{2} \mathrm{O}_{2}(8 \mathrm{~mL})$ were added carefully. The reaction mixture was stirred for $30 \mathrm{~min}$ at $0^{\circ} \mathrm{C}$ and additionally $2 \mathrm{~h}$ at rt. Sat. aq. $\mathrm{NaCl}(20 \mathrm{~mL})$ was added and the organic layer was separated. The aqueous layer was extracted with EtOAc $(2 \times 100 \mathrm{~mL})$. The combined organic layer was washed with sat. aq. $\mathrm{NaCl}(20 \mathrm{~mL})$ and dried over $\mathrm{MgSO}_{4}$. The solvents were removed under reduced pressure. The residue was diluted with toluene $(2 \times 50 \mathrm{~mL})$ and the solvent was distilled off. The remaining crude alcohol (mixture of isomers) was used in the next step without further purification.

The crude alcohol was dissolved in $\mathrm{CH}_{2} \mathrm{Cl}_{2} / \mathrm{CH}_{3} \mathrm{CN}(10: 1,55 \mathrm{~mL})$ and molecular sieves (4 $\AA$, 2.70 g), NMO (2.08 g, $17.7 \mathrm{mmol}, 1.50 \mathrm{eq}$ ) and TPAP (42 mg, $0.12 \mathrm{mmol}, 0.01 \mathrm{eq}$ ) were added. After stirring for $16 \mathrm{~h}$ at $\mathrm{rt}$ the reaction mixture was poured into a mixture of sat. aq. $\mathrm{NH}_{4} \mathrm{Cl}$ $(50 \mathrm{~mL})$ and MTBE $(100 \mathrm{~mL})$. The organic layer was separated and the aqueous layer was extracted with MTBE $(2 \times 25 \mathrm{~mL})$. The combined organic layer was dried over $\mathrm{MgSO}_{4}$ and the solvents were removed under reduced pressure. The remaining crude product (r.r. 8:1) was purified by flash chromatography on silica (n-pentane/Et $2 \mathrm{O} 1: 1$ to 1:2) to give ketone $\mathbf{S} 3$ (r.r. > 25:1, $989 \mathrm{mg}, 4.35 \mathrm{mmol}, 37 \%$ over 2 steps) as a colorless oil. TLC (MTBE): $\mathrm{R}_{f}=0.30$. ${ }^{1} \mathbf{H}$ NMR (500 MHz, $\left.\mathrm{CDCl}_{3}\right): \delta=1.20\left(\mathrm{~s}, 3 \mathrm{H}, \mathrm{CH}_{3}\right), 1.51(\mathrm{ddd}, J=15.2,8.7,3.3 \mathrm{~Hz}, 2 \mathrm{H}, 3-\mathrm{H} a$, 8-Hb), 1.71-1.85 (m, 4H, 3-H, 7-H), 2.25 (ddd, $\left.J=15.2,8.9,3.3 \mathrm{~Hz}, 2 \mathrm{H}, 3-\mathrm{H}_{b}, 8-\mathrm{H}_{b}\right), 2.34$ (ddd, $J=12.5,7.0,5.4 \mathrm{~Hz}, 2 \mathrm{H}, 4-\mathrm{H}_{a}, 6-\mathrm{H}_{a}$ ), 2.56 (ddd, $J=12.5,9.1,5.6 \mathrm{~Hz}, 2 \mathrm{H}, 4-\mathrm{H}_{b}, 6-\mathrm{H}_{b}$ ), $3.18\left(\mathrm{~s}, 3 \mathrm{H}, \mathrm{NCH}_{3}\right), 3.66\left(\mathrm{~s}, 3 \mathrm{H}, \mathrm{OCH}_{3}\right) .{ }^{13} \mathrm{C} \mathrm{NMR}\left(125 \mathrm{MHz}, \mathrm{CDCl}_{3}\right): \delta=21.0(2 \mathrm{C}, \mathrm{C} 3+\mathrm{C} 7)$, $24.9\left(\mathrm{CH}_{3}\right), 34.0\left(\mathrm{NCH}_{3}\right), 34.1(2 \mathrm{C}, \mathrm{C} 2+\mathrm{C} 8), 43.8(2 \mathrm{C}, \mathrm{C} 4+\mathrm{C} 6), 46.3(\mathrm{C} 1), 60.6\left(\mathrm{OCH}_{3}\right)$, $178.2\left(\mathrm{CONCH}_{3} \mathrm{OCH}_{3}\right), 216.6(\mathrm{C} 5)$. HR-MS (ESI): $\mathrm{m} / \mathrm{z}$ calcd for $\mathrm{C}_{12} \mathrm{H}_{22} \mathrm{NO}_{3}[\mathrm{M}+\mathrm{H}]^{+}$: 228.1594; found: 228.1591. FT-IR (neat): $\tilde{v}=2936$ (m), 1699 (s), 1638 (s), 1465 (m), 1380 (w), 1352 (m), 1201 (m), 1111 (m), 993 (s), 751 (s), 665 (w), 558 (m), $511(\mathrm{w}), 428(\mathrm{~m})$.

\section{(E)-5-(Methoxy(methyl)carbamoyl)-5-methylcyclooct-1-en-1-yl}

trifluoromethanesulfonate (S4)
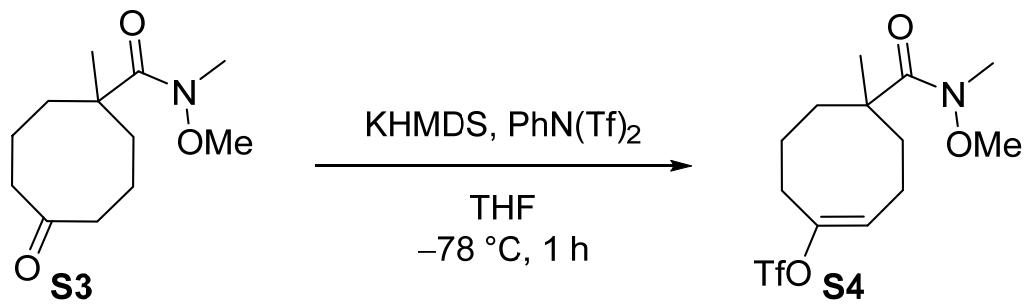

Ketone S3 (1.64 g, $7.21 \mathrm{mmol}, 1.00 \mathrm{eq})$ and $\mathrm{PhN}(\mathrm{Tf}) 2(2.71 \mathrm{~g}, 7.58 \mathrm{mmol}, 1.05 \mathrm{eq})$ were dissolved in THF $(30 \mathrm{~mL})$ and cooled to $-78{ }^{\circ} \mathrm{C}$. KHMDS $(0.5 \mathrm{M}$ in toluene, $15.9 \mathrm{~mL}$, 
$7.94 \mathrm{mmol}, 1.10 \mathrm{eq}$ ) was added dropwise over $10 \mathrm{~min}$. The resulting mixture was stirred for $1 \mathrm{~h}$ at $-78{ }^{\circ} \mathrm{C}$ and was then quenched by addition of sat. aq. $\mathrm{NaHCO}_{3}(20 \mathrm{~mL})$. The mixture was extracted with MTBE $(2 \times 30 \mathrm{~mL})$ and the combined organic layer was washed with sat. aq. $\mathrm{NaCl}(1 \times 20 \mathrm{~mL})$ and dried over $\mathrm{MgSO}_{4}$. The solvents were removed under reduced pressure. The remaining crude product was purified by column chromatography on silica (n-pentane/MTBE 2:1) to give vinyl triflate S4 (2.50 g, $6.96 \mathrm{mmol}, 96 \%)$ as a colorless oil. TLC (n-hexane/MTBE 1:1): $\mathrm{R}_{f}=0.26 .{ }^{1} \mathbf{H}$ NMR $\left(500 \mathrm{MHz}, \mathrm{CDCl}_{3}\right): \delta=1.25\left(\mathrm{~s}, 3 \mathrm{H}, \mathrm{CH}_{3}\right)$, 1.42, (ddd, $\left.J=14.9,9.4,1.8 \mathrm{~Hz}, 1 \mathrm{H}, 4-\mathrm{H}_{a}\right), 1.64-1.72$ (m, 2H, 6- $\left.\mathrm{H} a, 7-\mathrm{H}_{a}\right), 1.75-1.84$ (m, 1H, 6- $\left.\mathrm{H}_{b}\right), 2.08-2.25\left(\mathrm{~m}, 2 \mathrm{H}, 3-\mathrm{H}_{a}, 7-\mathrm{H}_{\mathrm{b}}\right), 2.31$ (ddd, $\left.J=16.1,5.5,5.5 \mathrm{~Hz}, 1 \mathrm{H}, 8-\mathrm{H}_{a}\right), 2.46-2.56$ (m, 2H, 3- $\left.\mathrm{H}_{b}, 4-\mathrm{H}_{b}\right), 2.73$ (ddd, $\left.J=16.0,10.6,5.3 \mathrm{~Hz}, 1 \mathrm{H}, 8-\mathrm{H}_{b}\right), 3.20$ (s, 3H, NCH ), 3.69 (s, $\left.3 \mathrm{H}, \mathrm{NOCH}_{3}\right), 5.82(\mathrm{t}, J=6.6 \mathrm{~Hz}, 1 \mathrm{H}, 2-\mathrm{H}) .{ }^{13} \mathrm{C} \mathrm{NMR}\left(125 \mathrm{MHz}, \mathrm{CDCl}_{3}\right): \delta=23.9(\mathrm{C} 3), 23.5$ (C7), $25.7\left(\mathrm{CH}_{3}\right), 29.4(\mathrm{C} 8), 31.7(\mathrm{C} 6), 33.9\left(\mathrm{NCH}_{3}\right), 36.2(\mathrm{C} 4), 46.6(\mathrm{C} 5), 60.6\left(\mathrm{NOCH}_{3}\right)$, $118.7\left(\mathrm{q}, J=320 \mathrm{~Hz}, \mathrm{CF}_{3}\right), 123.2(\mathrm{C} 2), 148.9(\mathrm{C} 1), 178.1\left(\mathrm{CONCH}_{3} \mathrm{OCH}_{3}\right),{ }^{19} \mathbf{F} \mathbf{~ N M R}$ $\left(282 \mathrm{MHz}, \mathrm{CDCl}_{3}\right): \delta=-75.0$. HR-MS (ESI): $\mathrm{m} / \mathrm{z}$ calcd for $\mathrm{C}_{13} \mathrm{H}_{20} \mathrm{~F}_{3} \mathrm{NO}_{5} \mathrm{SNa}[\mathrm{M}+\mathrm{Na}]^{+}$: 382.0906; found: 382.0901. FT-IR (neat): $\widetilde{v}=2941(\mathrm{w}), 1643(\mathrm{~m}), 1465(\mathrm{w}), 1412(\mathrm{~m})$, $1365(\mathrm{w}), 1206$ (s), 1143 (s), 997 (m), 954 (w), $935(\mathrm{w}), 900(\mathrm{w}), 864(\mathrm{w}), 844(\mathrm{w}), 614(\mathrm{w})$.

\section{(E)-5-(Hydroxymethyl)-5-methylcyclooct-1-en-1-yl trifluoromethanesulfonate (8)}

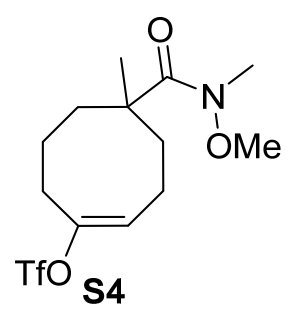

1) $\mathrm{LiAlH}_{4}, \mathrm{THF} / \mathrm{Et}_{2} \mathrm{O},-20^{\circ} \mathrm{C}, 10 \mathrm{~min}$

2) $\mathrm{NaBH}_{4}, \mathrm{MeOH}, 0^{\circ} \mathrm{C}, 15 \mathrm{~min}$

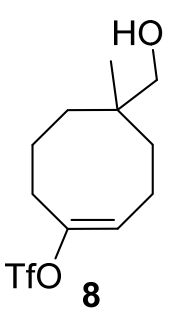

Vinyl triflate S4 (3.40 g, $9.46 \mathrm{mmol}, 1.00 \mathrm{eq})$ was dissolved in THF/Et $2 \mathrm{O}(2: 1,60 \mathrm{~mL})$ and cooled to $-20{ }^{\circ} \mathrm{C} . \mathrm{LiAlH}_{4}(359 \mathrm{mg}, 9.46 \mathrm{mmol}, 1.00 \mathrm{eq})$ was added in portions. After stirring for $10 \mathrm{~min}$ at $-20^{\circ} \mathrm{C}$, a sat. aq. solution of sodium potassium tartrate $(50 \mathrm{~mL})$ was added. The resulting mixture was stirred for $1 \mathrm{~h}$ at $\mathrm{rt}$ and then $\mathrm{Et}_{2} \mathrm{O}(30 \mathrm{~mL})$ was added. The organic layer was separated and the aqueous layer was extracted with $\mathrm{Et}_{2} \mathrm{O}(1 \times 50 \mathrm{~mL})$. The combined organic layer was dried over $\mathrm{MgSO}_{4}$ and the solvents were removed under reduced pressure. The residue was dissolved in $\mathrm{MeOH}(60 \mathrm{~mL})$ and at $0{ }^{\circ} \mathrm{C} \mathrm{NaBH}_{4}(429 \mathrm{mg}, 11.4 \mathrm{mmol}, 1.20 \mathrm{eq})$ was added. After stirring for $15 \mathrm{~min}$ at $0{ }^{\circ} \mathrm{C}$ the reaction was quenched by adding sat. aq. $\mathrm{NH}_{4} \mathrm{Cl}$ $(60 \mathrm{~mL})$. The mixture was extracted with MTBE $(3 \times 80 \mathrm{~mL})$. The combined organic layer was dried over $\mathrm{MgSO}_{4}$ and the solvents were removed under reduced pressure. The remaining crude 
product was purified by column chromatography on silica (n-pentane/MTBE 2:1) to give alcohol 8 (2.19 g, $7.23 \mathrm{mmol}, 76 \%$ over 2 steps $)$ as a colorless oil.

The analytical data are identical to those obtained from $\mathbf{7} \rightarrow \mathbf{8}$.

\section{Synthetic Procedures for the Preparation of Cyclooctyne 2}

(1 R,8 S,9 r, Z)-Ethyl bicyclo[6.1.0]non-4-ene-9-metyhlcarboxylate (exo-11) and (1 R,8 S,9 s, Z)-Ethyl bicyclo[6.1.0]non-4-ene-9-metyhlcarboxylate (endo-11)<smiles>CCOC(=O)C(C)=[W]</smiles>

14

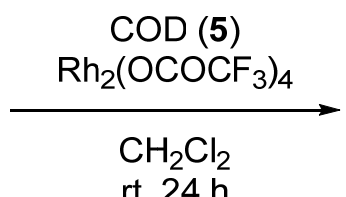

rt, $24 \mathrm{~h}$

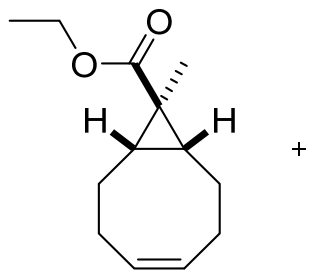

exo-11

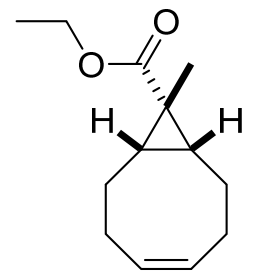

endo-11

To a solution of Rhodium (II) trifluoroacetate dimer (0.033 mmol, $21.8 \mathrm{mg}, 5.0 \mathrm{~mol} \%)$ and $\mathrm{COD}(3.25 \mathrm{~mL}, 26.5 \mathrm{mmol}, 4.00 \mathrm{eq})$ in $\mathrm{CH}_{2} \mathrm{Cl}_{2}(5.0 \mathrm{~mL})$ was added dropwise at rt over a period of $2 \mathrm{~h}$ a solution of diazo compound $14(0.85 \mathrm{~g}, 6.63 \mathrm{mmol}, 1.00 \mathrm{eq})$ in $\mathrm{CH}_{2} \mathrm{Cl}_{2}(5.0 \mathrm{~mL})$. After stirring for $24 \mathrm{~h}$ was the solvent removed in vaccuo and column chromatography on silica gel (n-pentane:MTBE; 30:1) gave products exo-11 (0.56 g, $2.70 \mathrm{mmol}, 41 \%$ ) and endo-11 $(0.033 \mathrm{~g}, 0.16 \mathrm{mmol}, 2 \%)$ as colorless oils.

Analytical data for (1 R,8 S,9 r, Z)-Ethyl bicyclo[6.1.0]non-4-ene-9methylcarboxylate (exo-11): TLC (n-pentane/MTBE 30:1): $\mathrm{R}_{f}=0.45 .{ }^{1} \mathbf{H}$ NMR $(500 \mathrm{MHz}$, $\left.\mathrm{CDCl}_{3}\right): \delta=5.61(\mathrm{mc}, 2 \mathrm{H}, 3,4-\mathrm{H}), 4.08\left(\mathrm{q}, 2 \mathrm{H},{ }^{3} \mathrm{~J}=7.1 \mathrm{~Hz}, \mathrm{CH}_{2} \mathrm{CH}_{3}\right), 2.40-2.34(\mathrm{~m}, 2 \mathrm{H}$, 2,5- $\left.H_{a}\right), 2.13-2.06\left(\mathrm{~m}, 2 \mathrm{H}, 2,5-\mathrm{H}_{b}\right), 1.96-1.89$ (m, 2H, 1,6- $\left.\mathrm{H}_{a}\right), 1.66-1.59$ (m, 2H, 1,6- $\left.\mathrm{H}_{b}\right), 1.57-$ $1.54(\mathrm{~m}, 2 \mathrm{H}, 7,8-\mathrm{H}), 1.23\left(\mathrm{t}, 3 \mathrm{H},{ }^{3} \mathrm{~J}=7.1 \mathrm{~Hz}, \mathrm{CH}_{2} \mathrm{CH}_{3}\right), 1.17(\mathrm{~s}, 3 \mathrm{H}, 10-\mathrm{H}) .{ }^{13} \mathrm{C}$ NMR (125 MHz, $\left.\mathrm{CDCl}_{3}\right): \delta=177.0(\mathrm{CO}), 129.3(3,4-\mathrm{C}), 60.5\left(\mathrm{CH}_{2} \mathrm{CH}_{3}\right), 29.0$ (7,8-C), 27.3 (2,5-C), 24.9 (9-C), 23.8 (1,6-C), $14.4\left(\mathrm{CH}_{2} \mathrm{CH}_{3}\right), 9.8$ (10-C). HR-MS (ESI+): $\mathrm{m} / \mathrm{z}$ calcd for $\mathrm{C}_{14} \mathrm{H}_{24} \mathrm{O}_{3}$ $[\mathrm{M}]^{+}$: for $\mathrm{C}_{13} \mathrm{H}_{20} \mathrm{O}_{2} \mathrm{H}: 209.1536[\mathrm{M}+\mathrm{H}]^{+}$; found: 209.1538 .

Analytical data for (1 $\mathrm{R}, 8 \quad \mathrm{~S}, 9 \quad \mathrm{~s}$, Z)-Ethyl bicyclo[6.1.0]non-4-ene-9methylcarboxylate (endo-11): TLC (n-pentane/MTBE 30:1): $\mathrm{R}_{f}=0.41 .{ }^{1} \mathbf{H}$ NMR $(600 \mathrm{MHz}$, $\left.\mathrm{CDCl}_{3}\right): \delta=5.58(\mathrm{mc}, 2 \mathrm{H}, 3,4-\mathrm{H}), 4.13\left(\mathrm{q}, 2 \mathrm{H},{ }^{3} \mathrm{~J}=7.1 \mathrm{~Hz}, \mathrm{CH}_{2} \mathrm{CH}_{3}\right), 2.48\left(\mathrm{mc}, 2 \mathrm{H}, 2,5-\mathrm{H}_{a}\right)$, 2.16-2.09 (m, 2H, 1,6- $\left.H_{a}\right), 2.05-1.99\left(\mathrm{~m}, 2 \mathrm{H}, 2,5-\mathrm{H}_{b}\right), 1.88-1.83\left(\mathrm{~m}, 2 \mathrm{H}, 1,6-\mathrm{H}_{b}\right), 1.27$ (s, 3H, 10-H), $1.26\left(\mathrm{t}, 3 \mathrm{H},{ }^{3} \mathrm{~J}=7.1 \mathrm{~Hz}, \mathrm{CH}_{2} \mathrm{CH}_{3}\right), 1.13-1.09$ (m, 2H, 7,8-H). ${ }^{13} \mathrm{C}$ NMR $(150 \mathrm{MHz}$, $\left.\mathrm{CDCl}_{3}\right): \delta=173.7(\mathrm{CO}), 129.5(3,4-C), 60.0\left(\mathrm{CH}_{2} \mathrm{CH}_{3}\right), 32.8(7,8-C), 27.3$ (2,5-C), $26.6(9-C)$, $24.0(10-\mathrm{C}), 23.6(1,6-\mathrm{C}), 14.5\left(\mathrm{CH}_{2} \mathrm{CH}_{3}\right)$. HR-MS (ESI): $\mathrm{m} / \mathrm{z}$ calcd for for $\mathrm{C}_{13} \mathrm{H}_{20} \mathrm{O}_{2} \mathrm{H}$ : 209.1536 [M+H] $]^{+}$; found: 209.1537. Analytical data for both diastereomers: FT-IR (neat): 
$\widetilde{v}=2972(\mathrm{w}), 2935(\mathrm{w}), 2876(\mathrm{w}), 2255(\mathrm{w}), 1713(\mathrm{~m}), 1462(\mathrm{w}), 1446(\mathrm{w}), 1385(\mathrm{w})$, 1367 (w), 1328 (m), 1257 (w), 1234 (w), 1203 (m), 1175 (s), 1129 (s), 1095 (w), 1028 (w), $951(\mathrm{w}), 909(\mathrm{~m}), 863(\mathrm{w}), 793(\mathrm{w}), 730(\mathrm{~s}), 671(\mathrm{w}), 648(\mathrm{w}), 621(\mathrm{w}), 571(\mathrm{w}), 526(\mathrm{w})$, $480(\mathrm{w})$.

(1 R,8 S,9 r)-4,5-dibromo-9-methylbicyclo[6.1.0]non-9-methanol (12):
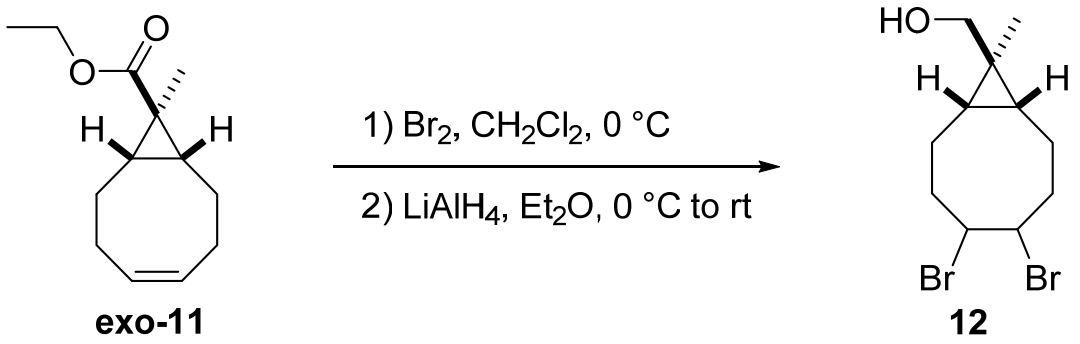

To a solution of olefin exo-11 (1.11 g, $5.33 \mathrm{mmol}, 1.00 \mathrm{eq})$ in $\mathrm{CH}_{2} \mathrm{Cl}_{2}(7.0 \mathrm{~mL})$ was added a solution of bromine $(0.38 \mathrm{~mL}, 7.47 \mathrm{mmol}, 1.40 \mathrm{eq})$ in $\mathrm{CH}_{2} \mathrm{Cl}_{2}(3.0 \mathrm{~mL})$ dropwise at $0{ }^{\circ} \mathrm{C}$. The reaction mixture was quenched after 30 min with a $20 \%$ aq. $\mathrm{Na}_{2} \mathrm{~S}_{2} \mathrm{O}_{4}$ solution, extracted with $\mathrm{CH}_{2} \mathrm{Cl}_{2}(2 \times 10 \mathrm{~mL})$ and dried over $\mathrm{Na}_{2} \mathrm{SO}_{4}$. The solvents were removed under reduced pressure affording the dibromide $(1.96 \mathrm{~g}, 5.33 \mathrm{mmol}, 99 \%)$ as a white solid. TLC (n-pentane/MTBE 9:1): $\mathrm{R}_{f}=0.47 .{ }^{1} \mathbf{H}$ NMR (500 MHz, $\left.\mathrm{CDCl}_{3}\right): \delta=4.81-4.80(\mathrm{~m}, 2 \mathrm{H}, \mathrm{CHBr}), 4.10(\mathrm{q}, 2 \mathrm{H}$, $\left.{ }^{3} J=7.1 \mathrm{~Hz}, \mathrm{CH}_{2} \mathrm{CH}_{3}\right), 2.79-2.73\left(\mathrm{~m}, 1 \mathrm{H}, \mathrm{CH}_{2} \mathrm{CHBr}\right), 2.69-2.63\left(\mathrm{~m}, 1 \mathrm{H}, \mathrm{CH}_{2} \mathrm{CHBr}\right)$, 2.32-2.25 (m, $\left.1 \mathrm{H}, \mathrm{CH}_{2} \mathrm{CHBr}\right), 2.19-2.14\left(\mathrm{~m}, 1 \mathrm{H}, \mathrm{CH}_{2} \mathrm{CHBr}\right), 1.92-1.87$ (m, 1H, $\mathrm{HCCHCH}_{2}$ ), 1.85-1.80 (m, 1H, $\left.\mathrm{HCCHCH}_{2}\right), 1.74-1.69\left(\mathrm{~m}, 1 \mathrm{H}, \mathrm{HCCHCH}_{2}\right), 1.66-1.61$ (m, 1H, $\mathrm{HCCHCH}_{2}$ ), 1.59-1.46 (m, 2H, HCCH), $1.25\left(\mathrm{t}, 3 \mathrm{H},{ }^{3} \mathrm{~J}=7.1 \mathrm{~Hz}, \mathrm{CH}_{2} \mathrm{CH}_{3}\right), 1.21(\mathrm{~s}, 3 \mathrm{H}, 10-\mathrm{H}) .{ }^{13} \mathrm{C}$ NMR (125 MHz, $\left.\quad \mathrm{CDCl}_{3}\right): \delta=176.4(\mathrm{CO}), \quad 60.7\left(\mathrm{CH}_{2} \mathrm{CH}_{3}\right), \quad 56.2(\mathrm{CHBr}), \quad 53.3(\mathrm{CHBr})$, $34.9\left(\mathrm{CH}_{2} \mathrm{CHBr}\right), \quad 34.7\left(\mathrm{CH}_{2} \mathrm{CHBr}\right), 29.9\left(\mathrm{HCCHCH}_{2}\right), \quad 27.2\left(\mathrm{HCCHCH}_{2}\right), \quad 26.6\left(\mathrm{C}_{\text {quart }}\right)$, $20.2(\mathrm{HCCH}), 19.5(\mathrm{HCCH}), 14.4\left(\mathrm{CH}_{2} \mathrm{CH}_{3}\right), 8.9(10-\mathrm{C})$. m.p.: $87^{\circ} \mathrm{C}\left(\mathrm{CH}_{2} \mathrm{Cl}_{2}\right)$. HR-MS (ESI): $\mathrm{m} / \mathrm{z}$ calcd for $\mathrm{C}_{13} \mathrm{H}_{20} \mathrm{Br}_{2} \mathrm{O}_{2} \mathrm{Na}: 390.9701[\mathrm{M}+\mathrm{Na}]^{+}$; found: 390.9702 . FT-IR (neat): $\widetilde{v}=2976(\mathrm{w}), 2930$ (w), 1709 (s), 1447 (w), 1417 (w), 1366 (m), 1295 (w), $1269(\mathrm{~m})$, 1236 (m), 1197 (m), 1197 (m), 1180 (m), 1168 (m), 1151 (m), 1109 (m), 1081 (m), 1035 (w), $995(\mathrm{~m}), 973(\mathrm{w}), 921(\mathrm{~m}), 870(\mathrm{~m}), 846(\mathrm{w}), 812(\mathrm{w}), 762(\mathrm{~m}), 725(\mathrm{w}), 594(\mathrm{~s}), 515(\mathrm{~m})$, $473(w)$.

To a solution of $\mathrm{LiAlH}_{4}(0.24 \mathrm{~g}, 6.26 \mathrm{mmol}, 1.20 \mathrm{eq})$ in $\mathrm{Et}_{2} \mathrm{O}(5.0 \mathrm{~mL})$ was added dropwise a solution of the ester $(1.92 \mathrm{~g}, 5.22 \mathrm{mmol}, 1.00 \mathrm{eq})$ in $\mathrm{Et}_{2} \mathrm{O}(5.0 \mathrm{~mL})$ at $0{ }^{\circ} \mathrm{C}$. The reaction mixture was allowed to warm to $\mathrm{rt}$ and quenched after $1 \mathrm{~h}$ with water $(2.0 \mathrm{~mL})$ at $0{ }^{\circ} \mathrm{C}$, extracted with $\mathrm{Et}_{2} \mathrm{O}(2 \times 10 \mathrm{~mL})$ and dried over $\mathrm{Na}_{2} \mathrm{SO}_{4}$. The solvent was removed under reduced pressure affording alcohol 12 (1.63 g, $5.00 \mathrm{mmol}, 96 \%)$ as a colorless solid. TLC (n-pentane/EtOAc 1:1): $\mathrm{R}_{f}=0.75 .{ }^{1} \mathbf{H}$ NMR $\left(300 \mathrm{MHz}, \mathrm{CDCl}_{3}\right): \delta=4.82-4.80(\mathrm{~m}, 2 \mathrm{H}, \mathrm{CHBr}), 3.36(\mathrm{~d}$, 
${ }^{2} J=1.3 \mathrm{~Hz}, 2 \mathrm{H}, \mathrm{CH}_{2} \mathrm{OH}$ ), 2.75-2.60 (m, 2H, 2-H, $\mathrm{CH}_{2} \mathrm{CHBr}$ ), 2.30-2.10 (m, 2H, $\mathrm{CH}_{2} \mathrm{CHBr}$ ), 1.92-1.77 (m, 2H, $\left.\mathrm{CHCH}_{2} \mathrm{CH}_{2}\right), 1.60-1.38\left(\mathrm{~m}, 2 \mathrm{H}, \mathrm{CHCH}_{2} \mathrm{CH}_{2}\right), 1.31(\mathrm{~s}, 1 \mathrm{H}, \mathrm{OH}), 1.08(\mathrm{~s}, 3 \mathrm{H}$, $\left.\mathrm{CCH}_{3}\right), 0.91-0.75(\mathrm{~m}, 2 \mathrm{H}, \mathrm{HCCH}) .{ }^{13} \mathrm{C}$ NMR $\left(75 \mathrm{MHz}, \mathrm{CDCl}_{3}\right): \delta=73.8\left(\mathrm{CH}_{2} \mathrm{OH}\right)$, 56.6 (CHBr), $\quad 54.1(\mathrm{CHBr}), \quad 35.2\left(\mathrm{CH}_{2} \mathrm{CHBr}\right), \quad 35.0\left(\mathrm{CH}_{2} \mathrm{CHBr}\right), \quad 25.6\left(\mathrm{HCCHCH}_{2}\right)$, $24.7\left(\mathrm{HCCHCH}_{2}\right), 21.6\left(\mathrm{C}_{\text {quart }}\right), 20.6(\mathrm{HCCH}), 19.8(\mathrm{HCCH}), 10.6\left(\mathrm{CH}_{3}\right)$. m.p.: $65^{\circ} \mathrm{C}\left(\mathrm{Et}_{2} \mathrm{O}\right)$. HR-MS (EI): $m / z$ calcd for $\mathrm{C}_{11} \mathrm{H}_{18} \mathrm{Br}_{1} \mathrm{O}_{1}: 245.0536$ and $247.0516[\mathrm{M}+\mathrm{H}]^{+}$; found: 245.0536 and 247.0516. FT-IR (neat): $\widetilde{v}=3312$ (br, w), 2917 (m), $2866(\mathrm{w}), 1462(\mathrm{w}), 1424$ (w), 1260 (w), 1182 (w), 1079 (m), 1050 (m), 1028 (s), 1013 (s), 977 (m), 923 (w), 855 (w), 800 (w), $681(\mathrm{~s}), 540(\mathrm{~m}), 518(\mathrm{~m}), 495(\mathrm{~m})$.

\section{(1 R,8 S,9 r)-bicyclo[6.1.0]non-4-bromo-4-en-9-methylethynyl (13)}

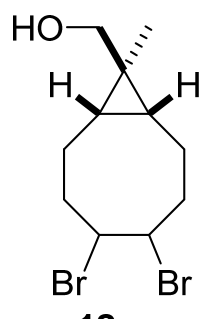

12
1) $\mathrm{KOtBu}, \mathrm{THF}, 0^{\circ} \mathrm{C}$

2) $\mathrm{DMP}, \mathrm{CH}_{2} \mathrm{Cl}_{2}$, rt

3) $10, \mathrm{~K}_{2} \mathrm{CO}_{3}, \mathrm{MeOH}$, rt

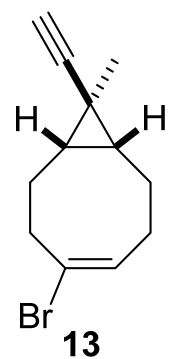

To a solution of alcohol $12(780 \mathrm{mg}, 2.39 \mathrm{mmol}, 1.00 \mathrm{eq})$ in THF $(5.0 \mathrm{~mL})$ was added dropwise a solution of $\mathrm{KO} \mathrm{Bu}^{\mathrm{B}}\left(5.02 \mathrm{~mL}, 2.10 \mathrm{eq}, 1 \mathrm{M}\right.$ in THF) at $0{ }^{\circ} \mathrm{C}$ over a period of $30 \mathrm{~min}$. The mixture was stirred $30 \mathrm{~min}$ at the same temperature and then quenched with aq. sat. $\mathrm{NH}_{4} \mathrm{Cl}$ $(10 \mathrm{~mL})$, extracted with $\mathrm{CH}_{2} \mathrm{Cl}_{2}(2 \times 10 \mathrm{~mL})$ and dried over $\mathrm{Na}_{2} \mathrm{SO}_{4}$. Column chromatography on silica gel (pent:MTBE; 2:1) gave the vinylbromide (396 mg, $1.61 \mathrm{mmol}, 67 \%$ ) as a yellow oil. TLC (n-pentane/MTBE 2:1): $\mathrm{R}_{f}=0.21 .{ }^{1} \mathbf{H}$ NMR $\left(500 \mathrm{MHz}, \mathrm{CDCl}_{3}\right): \delta=6.08-6.05(\mathrm{~m}$, $1 \mathrm{H}, \mathrm{BrC}=\mathrm{CH}), 3.34\left(\mathrm{~s}, 2 \mathrm{H}, \mathrm{CH}_{2} \mathrm{OH}\right), 2.94-2.88\left(\mathrm{~m}, 1 \mathrm{H}, \mathrm{CH}_{2}\right), 2.51-2.45\left(\mathrm{~m}, 1 \mathrm{H}, \mathrm{CH}_{2}\right), 2.38-$ $2.32\left(\mathrm{~m}, 1 \mathrm{H}, \mathrm{CH}_{2}\right), 2.13-2.03\left(\mathrm{~m}, 3 \mathrm{H}, \mathrm{CH}_{2}\right), 1.96-1.89\left(\mathrm{~m}, 1 \mathrm{H}, \mathrm{CH}_{2}\right), 1.64-1.53\left(\mathrm{~m}, 2 \mathrm{H}, \mathrm{CH}_{2}\right)$, $1.29(\mathrm{~s}, 1 \mathrm{H}, \mathrm{OH}), 1.04\left(\mathrm{~s}, 3 \mathrm{H}, \mathrm{CH}_{3}\right), 0.84-0.71(\mathrm{~m}, 2 \mathrm{H}, \mathrm{HCCH}) .{ }^{13} \mathrm{C}$ NMR $(125 \mathrm{MHz}$, $\left.\mathrm{CDCl}_{3}\right): \delta=131.1(\mathrm{HC}=\mathrm{CBr}), 125.2(\mathrm{HC}=\mathrm{CBr}), 73.8\left(\mathrm{CH}_{2} \mathrm{OH}\right), 38.1\left(\mathrm{CH}_{2} \mathrm{CBr}\right), 28.4\left(\mathrm{CH}_{2}\right)$, 24.5 ( $\left.\mathrm{C}_{\text {quart. }}\right), 23.5(\mathrm{HCCH}), 23.3(\mathrm{HCCH}), 23.2\left(\mathrm{CH}_{2}\right), 22.1\left(\mathrm{CH}_{2}\right), 11.4\left(\mathrm{CH}_{3}\right)$. HR-MS (ESI): m/z calcd for $\mathrm{C}_{11} \mathrm{H}_{16} \mathrm{OH}: 165.1276[\mathrm{M}+\mathrm{H}]^{+}$; found: 165.1274. FT-IR (neat): $\widetilde{v}=3327(\mathrm{w}, \mathrm{br})$, $2981(w), 2914$ (w), $2863(w), 1737(w), 1643$ (w), 1476 (w), $1428(w), 1385(w), 1228(w)$, $1137(\mathrm{w}), 1091(\mathrm{w}), 1025$ (s), 1004 (w), 955 (w), 888 (w), 834 (w), 796 (m), 754 (m), 701 (m), $663(\mathrm{~m}), 607(\mathrm{w}), 558(\mathrm{w}), 530(\mathrm{w})$.

To a solution of the alcohol $(0.37 \mathrm{~g}, 1.50 \mathrm{mmol}, 1.00 \mathrm{eq})$ in $\mathrm{CH}_{2} \mathrm{Cl}_{2}(7.0 \mathrm{~mL})$ was added $\operatorname{DMP}(1.27 \mathrm{~g}, 3.01 \mathrm{mmol}, 2.00 \mathrm{eq})$ at $\mathrm{rt}$ in one portion. After stirring for $1 \mathrm{~h}$, the reaction 
mixture was washed with aq. sat. $\mathrm{NaHCO}_{3}$, extracted with $\mathrm{CH}_{2} \mathrm{Cl}_{2}(2 \times 15 \mathrm{~mL})$ and dried over $\mathrm{Na}_{2} \mathrm{SO}_{4}$. The crude product was purified through a short column of silica gel (pent:MTBE; 5:1) affording the aldehyde (340 mg, $1.40 \mathrm{mmol}, 93 \%$ ) as a colorless oil. TLC ( $n$-pentane/MTBE 5:1): $\mathrm{R}_{f}=0.57 .{ }^{1} \mathbf{H}$ NMR $\left(500 \mathrm{MHz}, \mathrm{CDCl}_{3}\right): \delta=8.68$ (s, 1H, CHO), 6.13-6.09 (m, 1H, $\mathrm{BrC}=\mathrm{CH}), 2.99-2.94\left(\mathrm{~m}, 1 \mathrm{H}, \mathrm{CH}_{2} \mathrm{CBr}\right), 2.57-2.52\left(\mathrm{~m}, 1 \mathrm{H}, \mathrm{CH}_{2} \mathrm{CH}\right), 2.45-2.38(\mathrm{~m}, 1 \mathrm{H}$, $\mathrm{CH}_{2} \mathrm{CH}$ ), 2.19-2.10 (m, 2H, $\left.\mathrm{CH}_{2} \mathrm{CH}_{2} \mathrm{CH}\right), 2.02-1.95$ (m, $\left.1 \mathrm{H}, \mathrm{CH}_{2} \mathrm{CBr}\right), 1.75-1.64(\mathrm{~m}, 2 \mathrm{H}$, $\left.\mathrm{CH}_{2} \mathrm{CH}_{2} \mathrm{CBr}\right), 1.61-1.47$ (m, 2H, $\left.\mathrm{HCCH}\right), 1.66-1.61$ (m, 1H, $\left.\mathrm{HCCHCH}_{2}\right), 1.59-1.46(\mathrm{~m}, 2 \mathrm{H}$, $\mathrm{HCCH}), 1.15$ (s, 3H, $\left.\mathrm{CCH}_{3}\right) .{ }^{13} \mathrm{C}$ NMR $\left(125 \mathrm{MHz}, \mathrm{CDCl}_{3}\right): \delta=203.2(\mathrm{CO}), 130.8(\mathrm{BrC}=\mathrm{CH})$, $124.8(\mathrm{BrC}=\mathrm{CH}), \quad 37.8\left(\mathrm{CH}_{2} \mathrm{CBr}\right), \quad 34.5\left(\mathrm{C}_{\text {quart }}\right), \quad 27.9\left(\mathrm{CH}_{2} \mathrm{CH}_{2} \mathrm{CH}\right), \quad 26.4\left(\mathrm{CH}_{2} \mathrm{CH}_{2} \mathrm{CBr}\right)$, $25.5\left(\mathrm{CH}_{2} \mathrm{CH}_{2} \mathrm{CH}\right), 22.8(\mathrm{HCCH}), 22.5(\mathrm{HCCH}), 7.4\left(\mathrm{CCH}_{3}\right)$. HR-MS (EI): $\mathrm{m} / \mathrm{z}$ calcd for $\mathrm{C}_{11} \mathrm{H}_{15} \mathrm{BrOH}: 243.0382$ and $245.0362[\mathrm{M}+\mathrm{H}]^{+}$; found: 243.0379 and 245.0358 . FT-IR (neat): $\widetilde{v}=2926(\mathrm{w}), 2725(\mathrm{w}), 1698(\mathrm{~s}), 1643(\mathrm{w}), 1479(\mathrm{w}), 1434(\mathrm{w}), 1382(\mathrm{w}), 1319(\mathrm{w}), 1227(\mathrm{w})$, $1174(w), 1122(w), 1092(w), 1066(w), 1026(w), 974(w), 941(\mathrm{~m}), 892(w), 830(w), 752$ (w), $704(\mathrm{w}), 622(\mathrm{w}), 586(\mathrm{w}), 545(\mathrm{w}), 494(\mathrm{w})$.

To a solution of the aldehyde $(0.86 \mathrm{~g}, 3.53 \mathrm{mmol}, 1.00 \mathrm{eq})$ in $\mathrm{MeOH}(8.0 \mathrm{~mL})$ was added potassium carbonate $(1.46 \mathrm{~g}, 10.6 \mathrm{mmol}, 3.00 \mathrm{eq})$ in one portion and stirred for $15 \mathrm{~min}$ before adding Ohira-Bestmann reagent $10(1.02 \mathrm{~mL}, 4.23 \mathrm{mmol}, 1.20 \mathrm{eq})$ to the reaction mixture. After stirring for $45 \mathrm{~min}$ at $\mathrm{rt}$ aq. sat. $\mathrm{NaHCO}_{3}(10.0 \mathrm{~mL})$ was added and extracted with $\mathrm{Et}_{2} \mathrm{O}$ (2 x $15 \mathrm{~mL})$. Column chromatography on silica gel (pent:Et $2 \mathrm{O} ; 100: 1)$ gave alkyne $13(0.815 \mathrm{~g}$, $3.41 \mathrm{mmol}, 97 \%$ ) as a colorless liquid. TLC (n-pentane/Et ${ }_{2} \mathrm{O}$ 100:1): $\mathrm{R}_{f}=0.55 .{ }^{1} \mathbf{H}$ NMR (500 MHz, $\left.\mathrm{CDCl}_{3}\right): \delta=6.08-6.15(\mathrm{~m}, 1 \mathrm{H}, \mathrm{CH}=\mathrm{CBr}), 2.91-2.85\left(\mathrm{~m}, 1 \mathrm{H}, \mathrm{CH}_{2}\right), 2.52-2.46(\mathrm{~m}$, $\left.1 \mathrm{H}, \mathrm{CH}_{2}\right), 2.36-2.31\left(\mathrm{~m}, 1 \mathrm{H}, \mathrm{CH}_{2}\right), 2.20-2.13\left(\mathrm{~m}, 1 \mathrm{H}, \mathrm{CH}_{2}\right), 2.11-1.98\left(\mathrm{~m}, 2 \mathrm{H}, \mathrm{CH}_{2}\right), 1.84(\mathrm{~s}$, 1H, $\left.\mathrm{CCH}_{\text {alkyne }}\right), 1.63-1.49(\mathrm{~m}, 2 \mathrm{H}, \mathrm{HCCH}), 1.37-1.31\left(\mathrm{~m}, 1 \mathrm{H}, \mathrm{CH}_{2}\right), 1.28-1.19\left(\mathrm{~m}, 1 \mathrm{H}, \mathrm{CH}_{2}\right)$, $1.15\left(\mathrm{~s}, 3 \mathrm{H}, \mathrm{CH}_{3}\right) \cdot{ }^{13} \mathrm{C}$ NMR $\left(125 \mathrm{MHz}, \mathrm{CDCl}_{3}\right): \delta=130.9(\mathrm{BrC}=\mathrm{CH}), 124.9(\mathrm{BrC}=\mathrm{CH})$, $92.8(\mathrm{CCCH}), \quad 62.5(\mathrm{CCCH}), \quad 37.8\left(\mathrm{CH}_{2} \mathrm{CBr}\right), \quad 28.3(\mathrm{HCCH}), \quad 28.1\left(\mathrm{CH}_{2} \mathrm{CH}_{2} \mathrm{CBr}\right)$, $27.3(\mathrm{HCCH}), 23.0\left(\mathrm{CH}_{2} \mathrm{CH}_{2} \mathrm{CH}\right), 22.8\left(\mathrm{CH}_{2} \mathrm{CH}_{2} \mathrm{CH}\right), 13.8\left(\mathrm{CH}_{3}\right), 12.5\left(\mathrm{C}_{\text {quart }}\right)$. Elem. Anal. calcd. for $\mathrm{C}_{12} \mathrm{H}_{15} \mathrm{Br}$ : C, 60.27; H, 6.32. Found: C, 60.72; H, 6.69. FT-IR (neat): $\widetilde{v}=3292(\mathrm{w})$, $2990(w), 2931(w), 2866(w), 2106(w), 1643(w), 1478(w), 1436(w), 1382(w), 1336(w)$, $1299(\mathrm{w}), 1248(\mathrm{w}), 1225(\mathrm{w}), 1182(\mathrm{w}), 1128(\mathrm{w}), 1091(\mathrm{w}), 1050(\mathrm{w}), 1024(\mathrm{w}), 996(\mathrm{w})$, $932(\mathrm{w}), 890(\mathrm{w}), 834(\mathrm{~m}), 792(\mathrm{w}), 751(\mathrm{w}), 730(\mathrm{w}), 703(\mathrm{w}), 639(\mathrm{~s}), 598(\mathrm{~s}), 569(\mathrm{~m})$, $533(\mathrm{~m}), 473(\mathrm{w}), 439(\mathrm{w}), 408(\mathrm{w})$. 
(1 R,8 S,9 r)- bicyclo[6.1.0]-9-ethynyl-9-methyl (2)
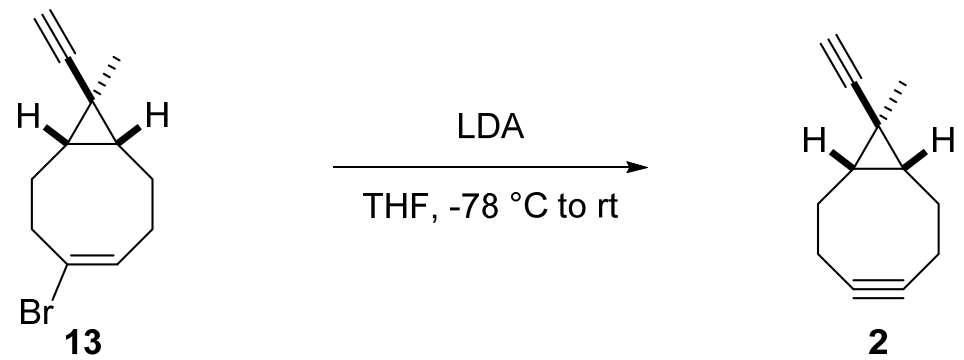

To a solution of vinyl bromide $13(0.276 \mathrm{~g}, 1.16 \mathrm{mmol}, 1.00 \mathrm{eq})$ in THF (4.0 mL) was added at $-78{ }^{\circ} \mathrm{C}$ dropwise a solution of diisopropyl amine $(0.49 \mathrm{~mL}, 3.47 \mathrm{mmol}, 3.00 \mathrm{eq})$ and $n$-butyllithium (2.5 M in hexane; $1.39 \mathrm{~mL}, 3.47 \mathrm{mmol}, 3.00 \mathrm{eq}$ ) in THF (4.0 mL). The reaction mixture was stirred $1 \mathrm{~h}$ at the same temperature and stirred an additional hour at rt. After addition of water $(3.0 \mathrm{~mL})$ was the aqueous layer extracted using $\mathrm{Et}_{2} \mathrm{O}(2 \times 10 \mathrm{~mL})$ and dried over $\mathrm{NaSO}_{4}$. Column chromatography on silica gel (pent:Et ${ }_{2} \mathrm{O} ; 100: 1$ ) gave cyclooctyne 2 $(0.148 \mathrm{~g}, 0.938 \mathrm{mmol}, 81 \%)$ as a colorless liquid. TLC ( $n$-pentane/Et $2 \mathrm{O} 50: 1): \mathrm{R}_{f}=0.41 .{ }^{1} \mathbf{H}$ NMR $\left(300 \mathrm{MHz}, \mathrm{CDCl}_{3}\right): \delta=2.33-2.19\left(\mathrm{~m}, 8 \mathrm{H}, \mathrm{CH}_{2}\right), 1.87$ (s, 1H, CCHalkyne), 1.50-1.37 (m, 2H, $\mathrm{HCCH}), 1.18\left(\mathrm{~s}, 3 \mathrm{H}, \mathrm{CH}_{3}\right) .{ }^{13} \mathrm{C}$ NMR $\left(75 \mathrm{MHz}, \mathrm{C}_{6} \mathrm{D}_{6}\right): \delta=98.5$ (3,4-C), 65.9 (11-C), 63.1 (12-C), 29.9 (1,6-C), 28.7 (2,5-C), 20.9 (7,8-C), 15.6 (2,5-C), 13.5 (9-C). HR-MS (EI+): m/z calcd for $\mathrm{C}_{12} \mathrm{H}_{15}[\mathrm{M}]^{+}$: 159.1174; found: 159.1185. FT-IR (neat): $\widetilde{v}=3291(\mathrm{w}), 2933(\mathrm{w})$, $2916(\mathrm{w}), 2853$ (w), 2104 (w), 1684 (w), 1442 (w), 1383 (w), $1311(\mathrm{w}), 1246(\mathrm{w}), 1218(\mathrm{w})$, $1125(\mathrm{w}), 1044(\mathrm{w}), 984(\mathrm{w}), 954(\mathrm{w}), 917(\mathrm{w}), 900(\mathrm{w}), 804(\mathrm{~m}), 751(\mathrm{~s}), 663(\mathrm{~m}), 640(\mathrm{~m})$, $606(\mathrm{~m}), 587(\mathrm{~m}), 547(\mathrm{w}), 526(\mathrm{w}), 512(\mathrm{~m})$.

\section{Synthetic Procedures for the Preparation of Bisazide 3}

2-(4-(Hydroxymethyl)phenyl)propan-2-ol (22)

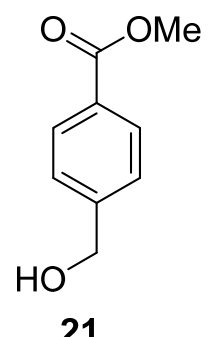

21

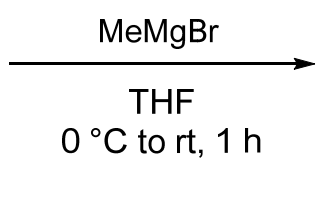

$\mathrm{HO}$

Methylbenzoate 21 (2.70 g, $16.2 \mathrm{mmol}, 1.00 \mathrm{eq})$ was dissolved in THF (100 mL) at $0{ }^{\circ} \mathrm{C}$. $\mathrm{MeMgBr}\left(3.0 \mathrm{M}\right.$ in $\left.\mathrm{Et}_{2} \mathrm{O}, 19.0 \mathrm{~mL}, 56.9 \mathrm{mmol}, 3.50 \mathrm{eq}\right)$ was added dropwise and the resulting mixture was stirred for $15 \mathrm{~min}$ at $0{ }^{\circ} \mathrm{C}$ and additionally for $1 \mathrm{~h}$ at $\mathrm{rt}$. The reaction was quenched by addition of sat. aq. $\mathrm{NH}_{4} \mathrm{Cl}(50 \mathrm{~mL})$ and extracted with EtOAc $(3 \times 50 \mathrm{~mL})$. The combined organic layer was dried over $\mathrm{MgSO}_{4}$ and the solvents were removed under reduced pressure. 
Purification by column chromatography on silica (n-pentane/EtOAc 1:1) gave diol 22 (2.60 g, $15.6 \mathrm{mmol}, 96 \%)$ as a colorless oil. TLC ( $n$-hexane/MTBE 1:2): $\mathrm{R}_{f}=0.17 .{ }^{1} \mathbf{H}$ NMR $\left(300 \mathrm{MHz} \mathrm{CDCl}_{3}\right): \delta=1.58\left(\mathrm{~s}, 6 \mathrm{H}, 2 \times \mathrm{CH}_{3}\right), 1.71(\mathrm{~s}, 2 \mathrm{H}, 2 \times \mathrm{OH}), 4.68\left(\mathrm{~s}, 2 \mathrm{H}, \mathrm{CH}_{2} \mathrm{OH}\right), 7.34$ $\left(\mathrm{d}, J=8.0 \mathrm{~Hz}, 2 \mathrm{H}, 3,5-\mathrm{H}_{A r}\right), 7.49$ (d, $\left.J=8.4 \mathrm{~Hz}, 2 \mathrm{H}, 2,6-\mathrm{H}_{A r}\right) .{ }^{13} \mathbf{C}$ NMR $(75 \mathrm{MHz}$, $\left.\mathrm{CDCl}_{3}\right): \delta=31.9\left(2 \mathrm{C}, 2 \times \mathrm{CH}_{3}\right), 65.2\left(\mathrm{CH}_{2}\right), 72.6\left(\mathrm{C}\left(\mathrm{CH}_{3}\right)_{2} \mathrm{OH}\right), 124.8\left(2 \mathrm{C}, \mathrm{CH}_{A r}\right), 127.1(2 \mathrm{C}$, $\left.\mathrm{CH}_{A r}\right), 139.5\left(\mathrm{C}_{A r}\right), 148.8$ (CAr). HR-MS (ESI): $\mathrm{m} / \mathrm{z}$ calcd for $\mathrm{C}_{10} \mathrm{H}_{14} \mathrm{O}_{2} \mathrm{Na}[\mathrm{M}+\mathrm{Na}]^{+}:$189.0886; found: 189.0886. FT-IR (neat): $\widetilde{v}=3320$ (br), 2973 (m), 2928 (w), 2871 (w), 1410 (m), 1362 (m), 1257 (m), 1213 (m), 1114 (m), 1095 (m), 1014 (s), 953 (s), 864 (m), 815 (s), 676 (m), $581(\mathrm{~m}), 550(\mathrm{~m})$.

\section{(4-(2-Azidopropan-2-yl)phenyl)methanol (3)}

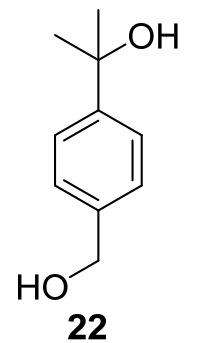

22

\section{1) $\mathrm{TMSN}_{3}, \mathrm{Bi}(\mathrm{OTf})_{3}, \mathrm{CH}_{2} \mathrm{Cl}_{2}$, rt 2) $\mathrm{MsCl}, \mathrm{NEt}_{3}, \mathrm{CH}_{2} \mathrm{Cl}_{2}$, rt}

3) $\mathrm{NaN}_{3}, \mathrm{DMSO}, \mathrm{rt}$

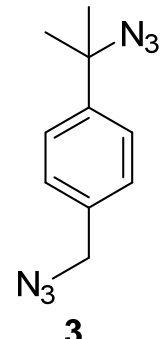

3

Diole 22 (327 mg, $1.97 \mathrm{mmol}, 1.00 \mathrm{eq})$ and $\mathrm{TMSN}_{3}(0.52 \mathrm{~mL}, 3.94 \mathrm{mmol}, 2.00 \mathrm{eq})$ were dissolved in $\mathrm{CH}_{2} \mathrm{Cl}_{2}(7.0 \mathrm{~mL})$. $\mathrm{Bi}(\mathrm{OTf})_{3}(6.5 \mathrm{mg}, 0.010 \mathrm{mmol}, 0.005 \mathrm{eq})$ was added and the resulting mixture was stirred at $\mathrm{rt}$ for $15 \mathrm{~min}$. The reaction was quenched by addition of sat. aq. $\mathrm{NaHCO}_{3}(5 \mathrm{~mL})$ and the organic layer was separated. The aqueous layer was extracted with $\mathrm{CH}_{2} \mathrm{Cl}_{2}(2 \times 10 \mathrm{~mL})$. The combined organic layer was dried over $\mathrm{MgSO}_{4}$ and the solvents were removed under reduced pressure. Purification by column chromatography on silica (n-pentane/MTBE 2:1) gave azide $(0.296 \mathrm{mg}, 1.55 \mathrm{mmol}, 79 \%)$ as a colorless oil. TLC (n-hexane/EtOAc 1:1): $\mathrm{R}_{f}=0.53 .{ }^{1} \mathbf{H}$ NMR $\left(500 \mathrm{MHz}, \mathrm{CDCl}_{3}\right): \delta=1.64\left(\mathrm{~s}, 6 \mathrm{H}, 2 \times \mathrm{CH}_{3}\right), 1.67$ (s, 1H, OH), 4.70 (s, 2H, $\left.\mathrm{CH}_{2} \mathrm{OH}\right), 7.37$ (d, $\left.J=8.3 \mathrm{~Hz}, 2 \mathrm{H}, 2,6-\mathrm{H}_{A r}\right), 7.44$ (d, J=8.3 Hz, 2H,

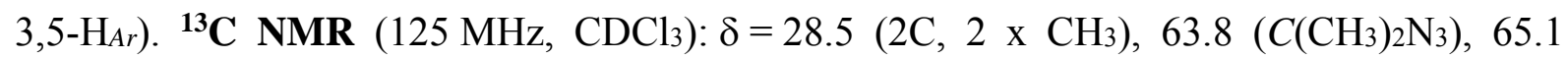
$\left(\mathrm{CH}_{2} \mathrm{OH}\right), 125.6\left(2 \mathrm{C}, \mathrm{C}_{A r} 3,5\right), 127.3$ (2C, $\left.\mathrm{C}_{A r} 2,6\right), 140.2\left(\mathrm{C}_{A r} 1\right), 144.3$ (C $\left.\mathrm{C}_{A r} 4\right)$. Elem. Anal. calcd. for $\mathrm{C}_{10} \mathrm{H}_{13} \mathrm{~N}_{3} \mathrm{O}: \mathrm{C}, 62.81 ; \mathrm{H}, 6.85 ; \mathrm{N}, 21.97$. Found: C, 62.35; H, 7.01; N, 21.96. FT-IR (neat): $\tilde{v}=3332$ (b), $2977(\mathrm{w}), 2931(\mathrm{w}), 2872(\mathrm{w}), 2100$ (s), $1511(\mathrm{w}), 1386(\mathrm{w}), 1257(\mathrm{~m})$, 1148 (m), 1045 (m), 960 (m), 818 (m), 721 (w), 627 (m).

The alcohol (315 mg, $1.65 \mathrm{mmol}, 1.00 \mathrm{eq})$ and NEt3 $(0.46 \mathrm{~mL}, 3.29 \mathrm{mmol}, 2.00 \mathrm{eq})$ were dissolved in $\mathrm{CH}_{2} \mathrm{Cl}_{2}(7.0 \mathrm{~mL})$. $\mathrm{MsCl}(197 \mathrm{mg}, 1.73 \mathrm{mmol}, 1.05 \mathrm{eq})$ was added dropwise at $0{ }^{\circ} \mathrm{C}$ and the reaction mixture was stirred at $\mathrm{rt}$ for $5 \mathrm{~h}$. The reaction was quenched by addition of $\mathrm{H}_{2} \mathrm{O}$ 
$(2 \mathrm{~mL})$ and sat. aq. $\mathrm{NaCl}(8 \mathrm{~mL})$ and the organic layer was separated. The aqueous layer was extracted with $\mathrm{CH}_{2} \mathrm{Cl}_{2}(1 \times 20 \mathrm{~mL})$. The combined organic layer was dried over $\mathrm{MgSO}_{4}$ and the solvent was removed under reduced pressure. The residue was dissolved in DMSO (7.0 mL) and $\mathrm{NaN}_{3}$ (214 mg, $3.29 \mathrm{mmol}, 2.00 \mathrm{eq}$ ) was added. After stirring for $16 \mathrm{~h}$ at $\mathrm{rt}$ the reaction mixture was diluted with $\mathrm{Et}_{2} \mathrm{O}$ and washed with $\mathrm{H}_{2} \mathrm{O}(2 \times 50 \mathrm{~mL})$ and sat. aq. $\mathrm{NaCl}(1 \times 20 \mathrm{~mL})$. The organic layer was dried over $\mathrm{MgSO}_{4}$ and the solvents were removed under reduced pressure. Column chromatography on silica (n-pentane/Et $2 \mathrm{O} 30: 1)$ gave bisazide 3 (100 mg, $0.46 \mathrm{mmol}, 28 \%$ ) as a colorless liquid.TLC ( $n$-hexane/MTBE 20:1): $\mathrm{R}_{f}=0.42 .{ }^{1} \mathbf{H}$ NMR $\left(500 \mathrm{MHz}, \mathrm{CDCl}_{3}\right): \delta=1.64\left(\mathrm{~s}, 6 \mathrm{H}, 2 \times \mathrm{CH}_{3}\right), 4.35\left(\mathrm{~s}, 2 \mathrm{H}, \mathrm{CH}_{2} \mathrm{~N}_{3}\right), 7.32(\mathrm{~d}, J=8.2 \mathrm{~Hz}, 2 \mathrm{H}$, 3,5-H $\left.\mathrm{H}_{A r}\right), 7.46\left(\mathrm{~d}, J=8.4 \mathrm{~Hz}, 2 \mathrm{H}, 2,6-\mathrm{H}_{A r}\right) .{ }^{13} \mathbf{C} \mathbf{N M R}\left(125 \mathrm{MHz}, \mathrm{CDCl}_{3}\right): \delta=28.5(2 \mathrm{C}$,

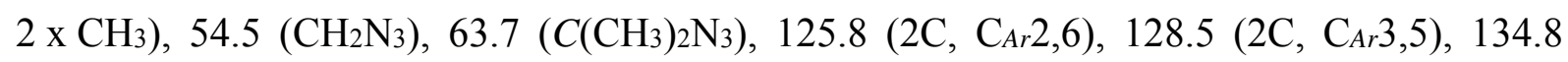
(C $\left.\mathrm{C}_{A r} 4\right), 145.0\left(\mathrm{C}_{A r} 1\right)$. HR-MS (EI): $\mathrm{m} / \mathrm{z}$ calcd for $\mathrm{C}_{10} \mathrm{H}_{12} \mathrm{~N}_{6}[\mathrm{M}]^{+}:$216.1124; found: 216.1123. FT-IR (neat): $\tilde{v}=2979(\mathrm{w}), 2933(\mathrm{w}), 2097$ (s), $1513(\mathrm{w}), 1343(\mathrm{w}), 1256(\mathrm{~m}), 1147$ (w), 1098 (w), $821(\mathrm{w}), 721(\mathrm{w}), 670(\mathrm{w}), 563(\mathrm{w})$.

\section{Studies on Model Systems}

\section{7-Ethynyl-7-methyl-5,6,7,8,9,10-hexahydrobenzo[8]annulene (16)}

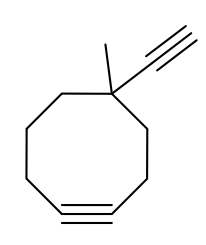

1<smiles>O=c1cccco1</smiles>

15

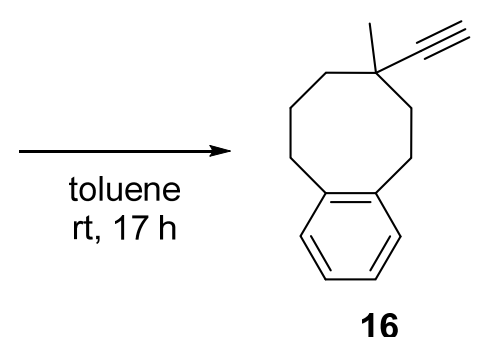

16

To a solution of cyclooctyne 1 (142 $\mathrm{mg}, 0.97 \mathrm{mmol}, 1.00 \mathrm{eq})$ in toluene (3.0 mL) was added 2-pyrone (15) (103 mg, $1.07 \mathrm{mmol}, 1.10 \mathrm{eq}$ ). After stirring for $17 \mathrm{~h}$ at $\mathrm{rt}$ the solvent was removed under reduced pressure and the residue was purified by column chromatography on silica ( $n$ pentane/Et $2 \mathrm{O} 50: 1)$ to give product 16 (148 $\mathrm{mg}, 0.75 \mathrm{mmol}, 76 \%)$ as a colorless liquid. TLC (n-hexane/MTBE 50:1): $\mathrm{R}_{f}=0.55 .{ }^{1} \mathbf{H}$ NMR $\left(600 \mathrm{MHz}, \mathrm{CDCl}_{3}\right): \delta=1.02$ (ddd, $J=13.8,13.8$, $\left.3.8 \mathrm{~Hz}, 1 \mathrm{H}, 8-\mathrm{H}_{a}\right), 1.21\left(\mathrm{~s}, 3 \mathrm{H}, \mathrm{CH}_{3}\right), 1.35$ (ddd, $\left.J=14.7,3.8,3.8 \mathrm{~Hz}, 1 \mathrm{H}, 8-\mathrm{H}_{b}\right), 1.42$ (ddd, $J$ $\left.=13.6,12.3,1.9 \mathrm{~Hz}, 1 \mathrm{H}, 6-\mathrm{H}_{a}\right), 1.55-1.62\left(\mathrm{~m}, 1 \mathrm{H}, 9-\mathrm{H}_{a}\right), 2.05-2.10\left(\mathrm{~m}, 1 \mathrm{H}, 6-\mathrm{H}_{b}\right), 2.10-2.17$

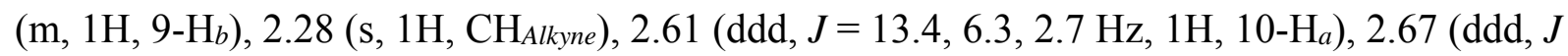
$\left.=14.3,7.2,2.0,1 \mathrm{H}, 5-\mathrm{H}_{a}\right), 3.09-3.16\left(\mathrm{~m}, 2 \mathrm{H}, 5-\mathrm{H}_{\mathrm{b}}, 10-\mathrm{H}_{b}\right), 7.06-7.09\left(\mathrm{~m}, 1 \mathrm{H}, \mathrm{H}_{A r}\right), 7.11-7.17$ (m, 3H, $\left.\mathrm{H}_{A r}\right) .{ }^{13} \mathrm{C}$ NMR (151 MHz, $\left.\mathrm{CDCl}_{3}\right): \delta=29.2$ (C9), $30.8(\mathrm{C} 10), 31.2(\mathrm{C} 5), 34.3\left(\mathrm{CH}_{3}\right)$, 35.0 (C8), 35.5 (C7), $46.6(\mathrm{C} 6), 71.0(\mathrm{CCH}), 89.4(\mathrm{CCH}), 126.3\left(\mathrm{CH}_{A r}\right), 126.6\left(\mathrm{CH}_{A r}\right), 129.0$ 
(C $\left.\mathrm{C}_{A r} 1\right), 129.4\left(\mathrm{C}_{A r} 4\right), 140.3\left(\mathrm{C}_{A r} 1^{\prime}\right), 142.3\left(\mathrm{C}_{A r} 4^{\prime}\right)$. HR-MS (EI): m/z calcd for $\mathrm{C}_{15} \mathrm{H}_{18}[\mathrm{M}]^{+}$: 198.1409; found: 198.1409. FT-IR (neat): $\widetilde{v}=3302$ (m), 2965 (m), 2928 (s), 2875 (m), 1492 (m), $1450(\mathrm{~m}), 757(\mathrm{~s}), 722(\mathrm{~m}), 629(\mathrm{~s}), 517(\mathrm{~m})$.

\section{1-Benzyl-4-(7-methyl-5,6,7,8,9,10-hexahydrobenzo[8]annulen-7-yl)-1H-1,2,3-triazole (19)}

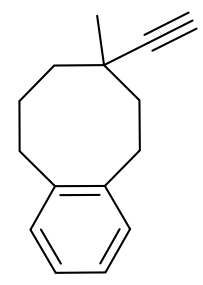

16

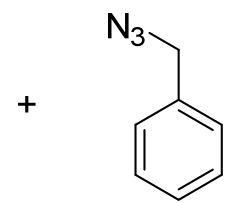

17

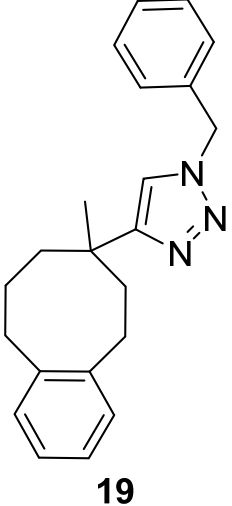

19

A solution of alkyne 16 (20 mg, $0.10 \mathrm{mmol}, 1.00 \mathrm{eq})$ and benzylazide (17) ${ }^{[2]}(16 \mathrm{mg}, 0.12 \mathrm{mmol}$, 1.20 eq $)$ in $t-\mathrm{BuOH} / \mathrm{H}_{2} \mathrm{O}(2: 1,1.5 \mathrm{~mL})$ was degassed by means of two freeze-pump-thaw cycles. Sodium L-ascorbate $(10 \mathrm{mg}, 0.05 \mathrm{mmol}, 0.50 \mathrm{eq})$ and $\mathrm{CuSO}_{4} \cdot 5 \mathrm{H}_{2} \mathrm{O}(5 \mathrm{mg}, 0.02 \mathrm{mmol}$, 0.20 eq) were added and the resulting mixture was stirred at $60^{\circ} \mathrm{C}$. After $2 \mathrm{~h} \mathrm{H}_{2} \mathrm{O}(5 \mathrm{~mL})$ was added and the mixture was extracted with $\mathrm{CH}_{2} \mathrm{Cl}_{2}(3 \times 10 \mathrm{~mL})$. The combined organic layer was dried over $\mathrm{MgSO}_{4}$. The solvent was removed under reduced pressure. Purification by column chromatography on silica ( $n$-pentane/EtOAc 2:1) gave triazole 19 (33 mg, $0.10 \mathrm{mmol}$, quant.) as a colorless oil. TLC ( $n$-hexane/EtOAc 4:1): $\mathrm{R}_{f}=0.09 .{ }^{1} \mathbf{H} \mathbf{N M R}\left(500 \mathrm{MHz}, \mathrm{CDCl}_{3}\right)$ : $\delta=1.30\left(\mathrm{~s}, 3 \mathrm{H}, \mathrm{CH}_{3}\right), 1.52\left(\mathrm{ddd}, J=14.7,8.4,4.2 \mathrm{~Hz}, 1 \mathrm{H}, 8-\mathrm{H}_{a}\right), 1.65(\mathrm{ddd}, J=14.7,8.2,3.9$ Hz, 1H, 8-Hb), 1.68-1.81 (m, 2H, 9-H), 1.89 (ddd, $J=14.2,9.4,3.0$ Hz, 1H, 6-Ha), 2.21 (ddd, $\left.J=14.2,9.2,3.0 \mathrm{~Hz}, 1 \mathrm{H}, 6-\mathrm{H}_{b}\right), 2.62$ (ddd, $J=13.6,7.5,6.1 \mathrm{~Hz}, 1 \mathrm{H}, 10-\mathrm{H}_{a}$ ), 2.70 (ddd, $J=13.6,7.5,6.1 \mathrm{~Hz}, 1 \mathrm{H}, 10-\mathrm{H} b), 2.85\left(\mathrm{ddd}, J=14.5,9.2,2.9 \mathrm{~Hz}, 1 \mathrm{H}, 5-\mathrm{H}_{a}\right), 2.93(\mathrm{ddd}, J=$ 14.5, 9.4, 2.9 Hz, 1H, 5-Hb), 5.48 (d, $J=3.5 \mathrm{~Hz}, 2 \mathrm{H}, \mathrm{NCH}_{2}$ ), 7.00-7.03 (m, 1H, $\mathrm{H}_{A r}$ ), 7.07 (s, 1H, $\mathrm{H}_{\text {Triazole }}$ ), 7.09-7.14 (m, 3H, $\left.\mathrm{H}_{A r}\right), 7.21-7.25$ (m, 2H, $\left.\mathrm{H}_{A r}\right), 7.32-7.40$ (m, 3H, $\left.\mathrm{H}_{A r}\right) .{ }^{13} \mathrm{C}$ NMR (125 MHz, $\mathrm{CDCl}_{3}$ ): $\delta=27.7$ (C9), 29.5 (CH3), 30.7 (C5), 31.8 (C10), 35.4 (C8), 37.9

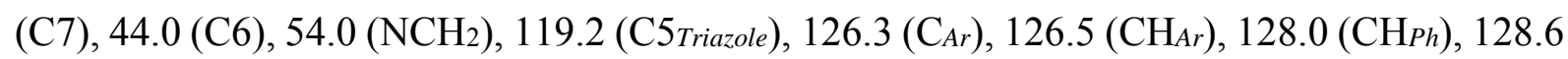
(CHPh), 129.09 (CHph), $129.1(\mathrm{C} 1), 129.4(\mathrm{C} 4), 135.3$ (CAr), 140.1 (CAr), 142.3 (CAr), 157.9 (C4 Triazole). HR-MS (ESI): $\mathrm{m} / z$ calcd for $\mathrm{C}_{22} \mathrm{H}_{25} \mathrm{~N}_{3} \mathrm{Na} \quad[\mathrm{M}+\mathrm{Na}]^{+}: 354.1941$; found: 354.1934 .

${ }^{[2]}$ L. S. Campbell-Verduyn, L. Mirfeizi, R. A. Dierckx, P. H. Elsinga, B. L. Feringa, Chem. Commun. 2009, 21392141. 
FT-IR (neat): $\widetilde{v}=2918$ (w), 2866 (m), 1492 (m), 1454 (m), 1218 (m), 1142 (w), 1048 (m), $910(\mathrm{~m}), 757$ (s), 712 (s), $694(\mathrm{~m}), 586(\mathrm{w}), 458(\mathrm{w})$.

4-(7-Methyl-5,6,7,8,9,10-hexahydrobenzo[8]annulen-7-yl)-1-(2-phenylpropan-2-yl)-1H1,2,3-triazole (20)

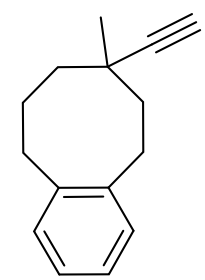

16

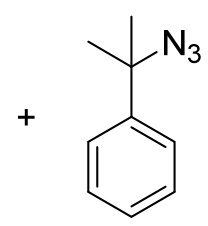

18

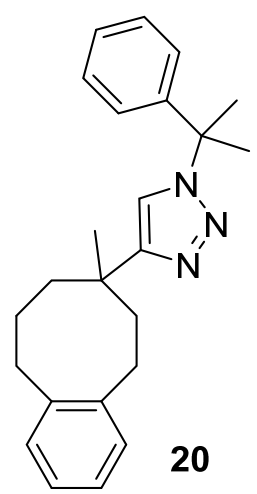

20

A solution of alkyne 16 (20 mg, $0.10 \mathrm{mmol}, 1.00 \mathrm{eq})$ and dimethylphenylazide (18) ${ }^{[3]}(20 \mathrm{mg}$, $0.12 \mathrm{mmol}, 1.20 \mathrm{eq})$ in $t-\mathrm{BuOH} / \mathrm{H}_{2} \mathrm{O}(2: 1,1.5 \mathrm{~mL})$ was degassed by means of two freeze-pumpthaw cycles. Sodium L-ascorbate $(10 \mathrm{mg}, 0.05 \mathrm{mmol}, 0.50 \mathrm{eq})$ and $\mathrm{CuSO}_{4} \cdot 5 \mathrm{H}_{2} \mathrm{O}(5 \mathrm{mg}$, $0.02 \mathrm{mmol}, 0.20 \mathrm{eq}$ ) were added and the resulting mixture was stirred at $80{ }^{\circ} \mathrm{C}$. After $19 \mathrm{~h} \mathrm{H}_{2} \mathrm{O}$ $(5 \mathrm{~mL})$ was added and the mixture was extracted with $\mathrm{CH}_{2} \mathrm{Cl}_{2}(3 \times 10 \mathrm{~mL})$. The combined organic layer was dried over $\mathrm{MgSO}_{4}$. The solvent was removed under reduced pressure. Purification by column chromatography on silica (n-pentane/EtOAc 2:1) gave triazole 20 (36 mg, 0.10 mmol, quant.) as a colorless oil. TLC ( $n$-hexane/EtOAc 4:1): $\mathrm{R}_{f}=0.19 .{ }^{1} \mathbf{H}$ NMR $\left(500 \mathrm{MHz} \mathrm{CDCl}_{3}\right): \delta=1.32\left(\mathrm{~s}, 3 \mathrm{H}, \mathrm{CH}_{3}\right), 1.52\left(\mathrm{ddd}, J=14.7,8.5,4.4 \mathrm{~Hz}, 1 \mathrm{H}, 8-\mathrm{H}_{a}\right), 1.65$ (ddd, $\left.J=14.7,8.0,4.0 \mathrm{~Hz}, 1 \mathrm{H}, 8-\mathrm{H}_{b}\right), 1.69-1.80$ (m, 2H, 9-H), 1.89 (ddd, $J=14.2,9.7,2.8 \mathrm{~Hz}$, $\left.1 \mathrm{H}, 6-\mathrm{H}_{a}\right), 2.03$ (s, 3H, C( $\left.\left.\mathrm{CH}_{3}\right)_{2} \mathrm{Ph}\right), 2.04$ (s, 3H, 3H, $\left.\mathrm{C}\left(\mathrm{CH}_{3}\right)_{2} \mathrm{Ph}\right), 2.24$ (ddd, J = 14.2, 9.2, 2.8 $\left.\mathrm{Hz}, 1 \mathrm{H}, 6-\mathrm{H}_{b}\right), 2.64\left(\mathrm{ddd}, J=13.8,7.1,6.8 \mathrm{~Hz}, 1 \mathrm{H}, 10-\mathrm{H}_{a}\right), 2.70(\mathrm{ddd}, J=13.4,6.9,6.6 \mathrm{~Hz}$, $\left.1 \mathrm{H}, 10-\mathrm{H}_{b}\right), 2.85\left(\mathrm{ddd}, J=14.5,9.2,2.8 \mathrm{~Hz}, 1 \mathrm{H}, 5-\mathrm{H}_{a}\right), 2.97$ (ddd, $J=14.5,9.7,2.8 \mathrm{~Hz}, 1 \mathrm{H}, 5-$ $\left.\mathrm{H}_{b}\right), 7.00-7.08\left(\mathrm{~m}, 4 \mathrm{H}, \mathrm{H}_{A r}\right), 7.08-7.14\left(\mathrm{~m}, 3 \mathrm{H}, \mathrm{H}_{A r}\right), 7.26-7.35\left(\mathrm{~m}, 3 \mathrm{H}, \mathrm{H}_{A r}\right) .{ }^{13} \mathbf{C}$ NMR

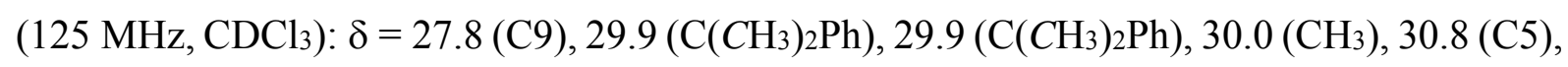

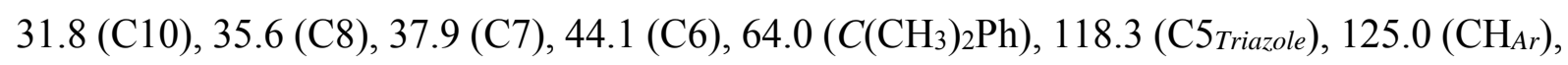
$126.3\left(\mathrm{CH}_{A r}\right), 126.5\left(\mathrm{CH}_{P h}\right), 127.8\left(\mathrm{CH}_{P h}\right), 128.8\left(\mathrm{CH}_{P h}\right), 129.1(\mathrm{C} 1), 129.4(\mathrm{C} 4), 140.2\left(\mathrm{C}_{A r}\right)$,

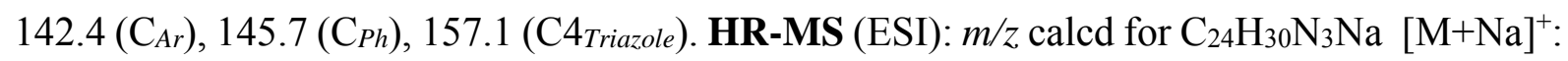
360.2434; found: 360.2439. FT-IR (neat): $\widetilde{v}=2923$ (m), $2867(\mathrm{w}), 1494$ (m), 1448 (m), $1389(\mathrm{w}), 1228(\mathrm{w}), 1039(\mathrm{~m}), 799(\mathrm{w}), 759(\mathrm{~s}), 734(\mathrm{w}), 699(\mathrm{~s})$.

${ }^{[3]}$ D. Balderman, A. Kalir, Synthesis 1978, 24-26. 


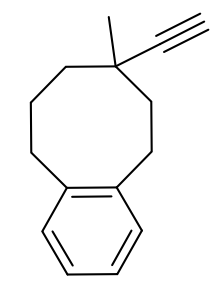

16

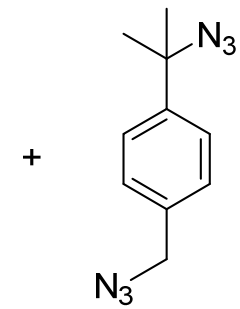

3

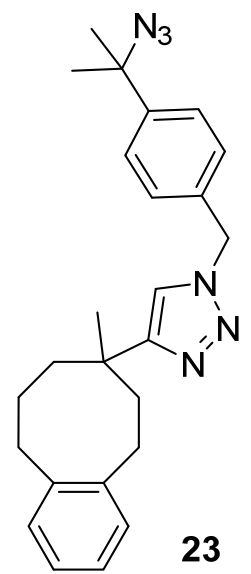

23

Alkyne 16 (20 mg, $0.10 \mathrm{mmol}, 1.00 \mathrm{eq})$ and bisazide 3 (26 mg, $0.12 \mathrm{mmol}, 1.20 \mathrm{eq})$ were dissolved in $t-\mathrm{BuOH} / \mathrm{H}_{2} \mathrm{O}(2: 1,1.5 \mathrm{~mL})$ and the solution was degassed by means of two freezepump-thaw cycles. Sodium L-ascorbate $(10 \mathrm{mg}, 0.05 \mathrm{mmol}, 0.50 \mathrm{eq})$ and $\mathrm{CuSO}_{4} \cdot 5 \mathrm{H}_{2} \mathrm{O}(5 \mathrm{mg}$, $0.02 \mathrm{mmol}, 0.20 \mathrm{eq}$ ) were added and the reaction mixture was stirred for $3 \mathrm{~h}$ at $60{ }^{\circ} \mathrm{C}$. Then $\mathrm{H}_{2} \mathrm{O}(5 \mathrm{~mL})$ was added and the mixture was extracted with $\mathrm{CH}_{2} \mathrm{Cl}_{2}(3 \times 10 \mathrm{~mL})$. The combined organic layers were dried over $\mathrm{MgSO}_{4}$ and the solvent was removed under reduced pressure. The remaining crude product was purified by column chromatography on silica (n-pentane/EtOAc 2:1) to yield triazole 23 (42 mg, $0.10 \mathrm{mmol}$, quant.) as a colorless gum. TLC (n-hexane/EtOAc 2:1): $\mathrm{R}_{f}=0.42 .{ }^{1} \mathbf{H}$ NMR $\left(500 \mathrm{MHz}, \mathrm{CDCl}_{3}\right): \delta=1.31\left(\mathrm{~s}, 3 \mathrm{H}, \mathrm{CH}_{3}\right), 1.53$ (ddd, $\left.J=14.8,8.4,4.2 \mathrm{~Hz}, 1 \mathrm{H}, 8-\mathrm{H}_{a}\right), 1.63\left(\mathrm{~s}, 6 \mathrm{H}, \mathrm{C}\left(\mathrm{CH}_{3}\right)_{2} \mathrm{~N}_{3}\right), 1.63-1.69$ (m, 1H, 8- $\left.\mathrm{H}_{b}\right), 1.69$ $1.83(\mathrm{~m}, 2 \mathrm{H}, 9-\mathrm{H}), 1.90\left(\mathrm{ddd}, J=14.2,9.3,3.0 \mathrm{~Hz}, 1 \mathrm{H}, 6-\mathrm{H}_{a}\right), 2.21(\mathrm{ddd}, J=14.2,9.3,3.0 \mathrm{~Hz}$, $\left.1 \mathrm{H}, 6-\mathrm{H}_{b}\right), 2.63\left(\mathrm{ddd}, J=13.5,6.7,6.7 \mathrm{~Hz}, 1 \mathrm{H}, 10-\mathrm{H}_{a}\right), 2.71$ (ddd, $J=13.6,7.5,6.1 \mathrm{~Hz}, 1 \mathrm{H}$, $\left.10-\mathrm{H}_{b}\right), 2.86\left(\mathrm{ddd}, J=14.5,9.3,3.0 \mathrm{~Hz}, 1 \mathrm{H}, 5-\mathrm{H}_{a}\right), 2.94(\mathrm{ddd}, J=14.5,9.3,3.0 \mathrm{~Hz}, 1 \mathrm{H}, 5-\mathrm{H} b)$, $5.47\left(\mathrm{~d}, J=3.4 \mathrm{~Hz}, 2 \mathrm{H}, \mathrm{CH}_{2} \mathrm{~N}\right.$ ), 6.99-7.04 (m, 1H, $\mathrm{H}_{A r}$ ), 7.07 (s, 1H, H $\left.3 \mathrm{H}, \mathrm{H}_{A r}\right), 7.21\left(\mathrm{~d}, J=8.3 \mathrm{~Hz}, 2 \mathrm{H}, 3,5-\mathrm{H}_{A r}\right), 7.44$ (d, $\left.J=8.3 \mathrm{~Hz}, 2 \mathrm{H}, 2,6-\mathrm{H}_{A r}\right) .{ }^{13} \mathbf{C}$ NMR (125 MHz, $\left.\mathrm{CDCl}_{3}\right): \delta=27.7$ (C9), $28.5\left(2 \mathrm{C}, \mathrm{C}\left(\mathrm{CH}_{3}\right)_{2} \mathrm{~N}_{3}\right), 29.4\left(\mathrm{CH}_{3}\right), 30.7$ (C5), $31.9(\mathrm{C} 10)$, $35.5(\mathrm{C} 8), 37.9(\mathrm{C} 6), 44.0(\mathrm{C} 7), 53.6\left(\mathrm{CH}_{2} \mathrm{~N}\right), 63.6\left(\mathrm{C}\left(\mathrm{CH}_{3}\right)_{2} \mathrm{~N}_{3}\right), 119.3\left(\mathrm{CH}_{\text {Triazole }}\right), 126.0(2 \mathrm{C}$, $\mathrm{C}_{A r}$ 2,6), $126.4\left(\mathrm{CH}_{A r}\right), 126.5\left(\mathrm{CH}_{A r}\right), 128.1\left(2 \mathrm{C}, \mathrm{C}_{A r} 3,5\right), 129.2\left(\mathrm{CH}_{A r}\right), 129.4\left(\mathrm{CH}_{A r}\right), 134.4$ (CAr), 140.1 ( $\left.\mathrm{C}_{A r}\right), 142.3$ (C $\left.\mathrm{C}_{A r}\right), 145.4$ (CAr1), 158.0 (C Triazole). HR-MS (ESI): m/z calcd for $\mathrm{C}_{25} \mathrm{H}_{30} \mathrm{~N}_{6} \mathrm{Na}[\mathrm{M}+\mathrm{Na}]^{+}:$437.2424; found: 437.2422. FT-IR (neat): $\widetilde{v}=2929(\mathrm{~m}), 2869(\mathrm{w})$, 2102 (s), 1491 (m), 1452 (w), 1416 (w), 1261 (m), 1145 (w), 1097 (w), 1049 (m), 816 (w), $758(\mathrm{~m}), 732(\mathrm{w}), 710(\mathrm{w})$. 


\section{Compound 24}
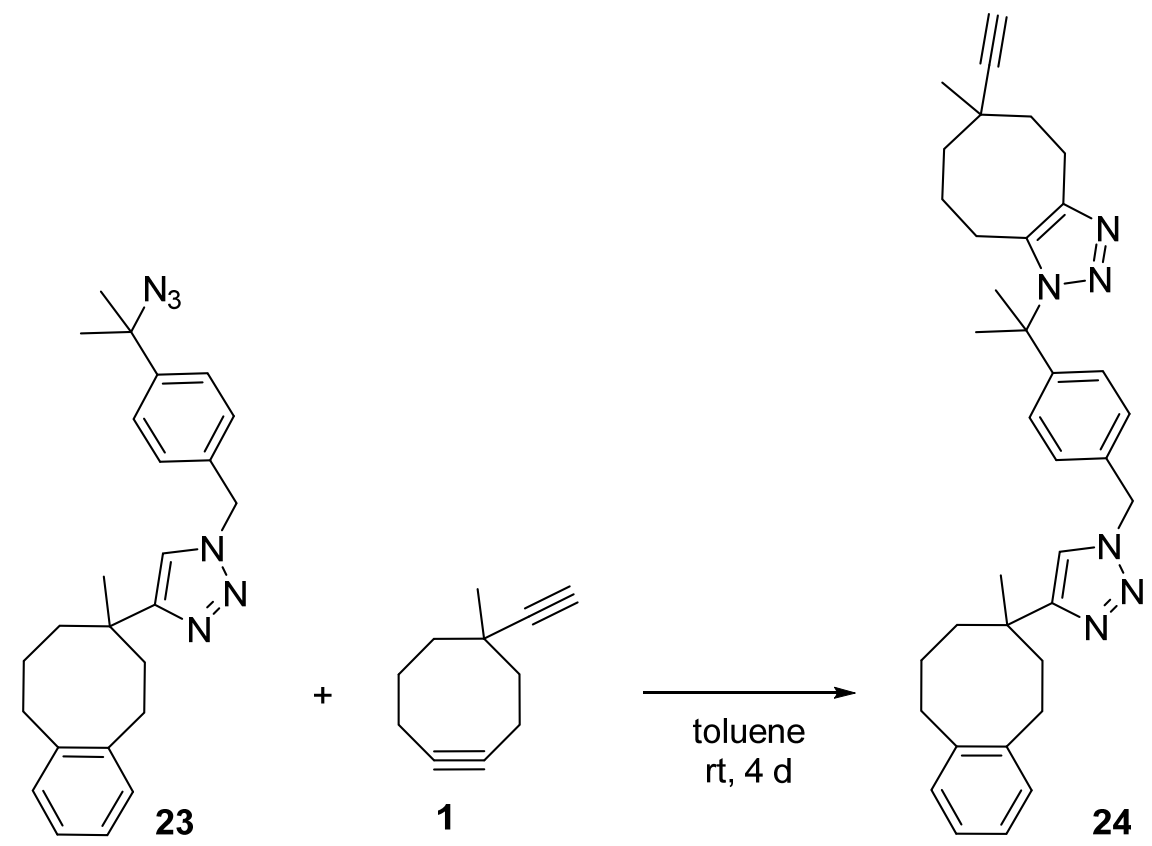

Azide 23 (30 mg, $0.72 \mathrm{mmol}, 1.00 \mathrm{eq})$ was dissolved in toluene (1.0 mL) and a solution of cyclooctyne 1 (12 $\mathrm{mg}, 0.81 \mathrm{mmol}, 1.10 \mathrm{eq})$ in toluene $(1.0 \mathrm{~mL})$ was added. The reaction was stirred at $\mathrm{rt}$ for $4 \mathrm{~d}$. The solvent was removed under reduced pressure and the remaining residue was purified by column chromatography on silica (n-pentane/EtOAc 2:3) to give compound 24 (mixture of regio- and diastereomers, $37 \mathrm{mg}, 0.067 \mathrm{mmol}, 93 \%$ ) as a colorless gum. Analytical data for mixture of isomeres: TLC (n-hexane/EtOAc 1:1): $\mathrm{R}_{f}=0.18 .{ }^{1} \mathbf{H}$ NMR $\left(500 \mathrm{MHz}, \mathrm{CDCl}_{3}\right): \delta=0.78-0.90(\mathrm{~m}, 1 \mathrm{H}), 1.01-1.10(\mathrm{~m}, 2 \mathrm{H}), 1.15-1.40(\mathrm{~m}, 6 \mathrm{H}), 1.46-1.82$ (m, 6H), 1.82-1.94 (m, 2H), 1.97-2.11 (m, 7H), 2.14-2.39 (m, 2H), 2.50-2.74 (m, 2H), 2.81$3.05(\mathrm{~m}, 5 \mathrm{H}), 5.45\left(\mathrm{~s}, 2 \mathrm{H}, \mathrm{CH}_{2} \mathrm{~N}\right), 6.99-7.04\left(\mathrm{~m}, 1 \mathrm{H}, \mathrm{H}_{A r}\right), 7.04-7.14\left(\mathrm{~m}, 6 \mathrm{H}, \mathrm{H}_{A r}\right), 7.14-7.20$ (m, 2H, $\left.\mathrm{H}_{A r}\right) .{ }^{13} \mathrm{C}$ NMR (125 MHz, $\left.\mathrm{CDCl}_{3}\right): \delta=21.3,21.8,22.5,23.3,23.8,24.1,27.7,29.0$, 29.5, 29.6, 29.7, 30.1, 30.3, 30.4, 30.7, 31.2, 31.8, 34.6, 34.8, 34.9, 35.0, 35.4, 36.4, 36.6, 37.9, 39.5, 44.0, $53.4\left(\mathrm{CH}_{2} \mathrm{~N}\right), 64.0,64.4,69.2,70.0,90.1,90.8,119.1,125.8,126.3,126.5,128.2$, $128.3,129.1,129.4,131.3,133.4,134.8,140.0,142.2$, 144.4, 145.4, 146.1, 147.3, 158.1. HRMS (ESI): $\mathrm{m} / \mathrm{z}$ calcd for $\mathrm{C}_{36} \mathrm{H}_{44} \mathrm{~N}_{6} \mathrm{Na}[\mathrm{M}+\mathrm{Na}]^{+}: 583.3520$; found: 583.3520. FT-IR (neat): $\widetilde{v}=3302(\mathrm{w}), 2930(\mathrm{~m}), 2858(\mathrm{w}), 2243(\mathrm{w}), 1674(\mathrm{w}), 1514(\mathrm{w}), 1451(\mathrm{~m}), 1250(\mathrm{~m}), 1049$ (m), $911(\mathrm{~m}), 854(\mathrm{w}), 729(\mathrm{~s}), 645(\mathrm{~m}), 587(\mathrm{w})$. 


\section{Compound 25}
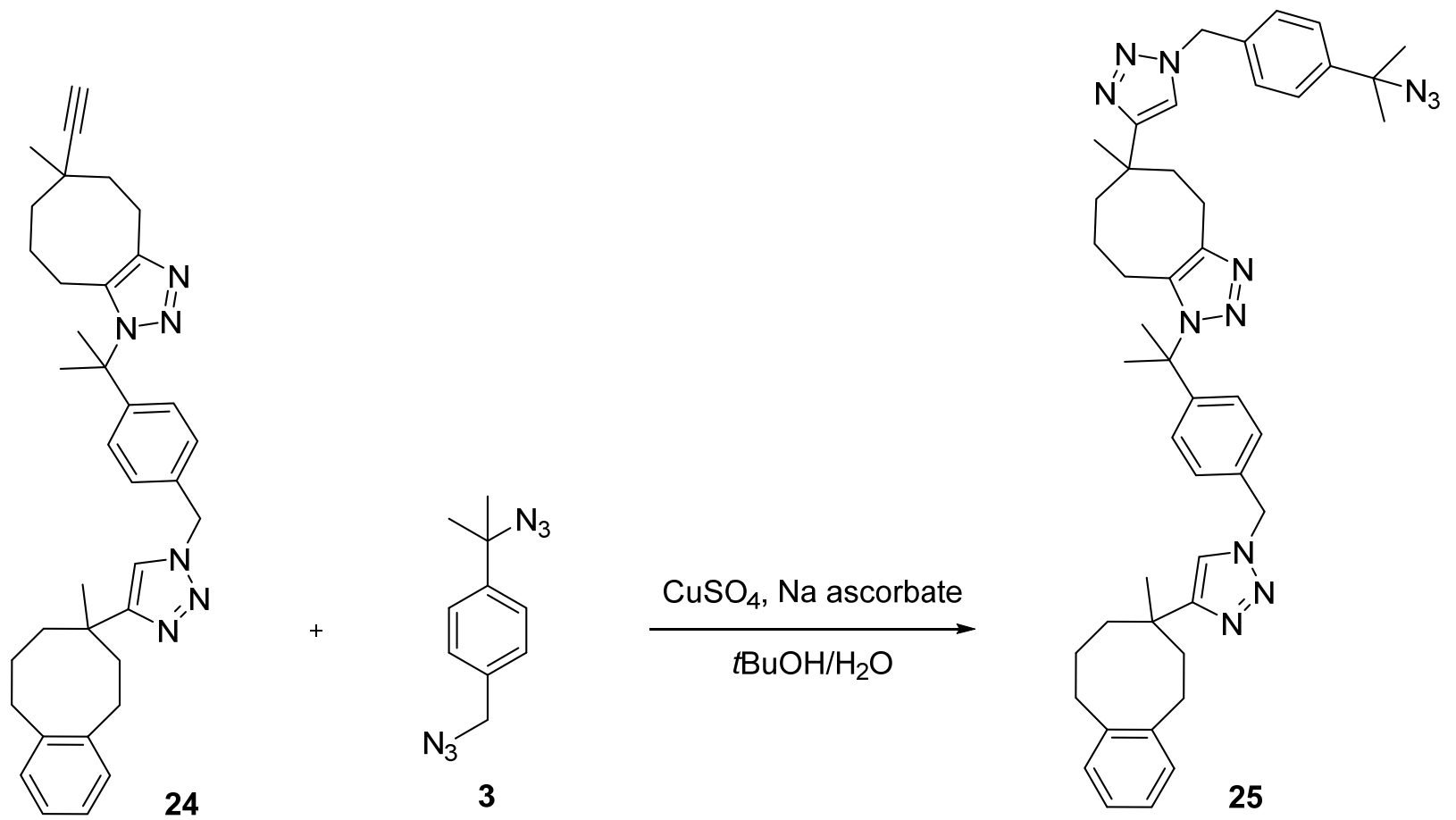

Alkyne 24 (29 mg, $0.053 \mathrm{mmol}, 1.00 \mathrm{eq}$ ) and bisazide 3 (14 mg, $0.063 \mathrm{mmol}, 1.20 \mathrm{eq}$ ) were dissolved in $t \mathrm{BuOH} / \mathrm{H}_{2} \mathrm{O}(2: 1,1.5 \mathrm{~mL})$ and the solution was degassed by means of freeze-pumpthaw cycles. Sodium L-ascorbat (5.2 mg, $0.026 \mathrm{mmol}, 0.50 \mathrm{eq})$ and $\mathrm{CuSO}_{4} \cdot 5 \mathrm{H}_{2} \mathrm{O}(2.6 \mathrm{mg}$, $0.011 \mathrm{mmol}, 0.20 \mathrm{eq}$ ) were added and the reaction mixture was stirred for $30 \mathrm{~min}$ at $60{ }^{\circ} \mathrm{C}$. Then $\mathrm{H}_{2} \mathrm{O}(5 \mathrm{~mL})$ was added and the mixture was extracted with $\mathrm{CH}_{2} \mathrm{Cl}_{2}(3 \times 10 \mathrm{~mL})$ and EtOAc $(3 \times 10 \mathrm{~mL})$. The combined organic layers were dried over $\mathrm{MgSO}_{4}$ and the solvent was removed under reduced pressure. The remaining crude product was purified by column chromatography on silica (EtOAc) to yield triazole 25 (mixture of regio- and diastereomers, 39 $\mathrm{mg}, 0.039 \mathrm{mmol}, 95 \%$ ) as a colorless gum. Analytical data for mixture of isomeres: TLC ( $n$ hexane/EtOAc 2:3): $\mathrm{R}_{f}=0.42 .{ }^{1} \mathbf{H}$ NMR $\left(500 \mathrm{MHz}, \mathrm{CDCl}_{3}\right): \delta=0.97-2.14(\mathrm{~m}, 31 \mathrm{H}, \mathrm{H}), 2.14$ $2.74(\mathrm{~m}, 5 \mathrm{H}, \mathrm{H}), 2.77-3.16(\mathrm{~m}, 4 \mathrm{H}, \mathrm{H}), 5.39-5.52$ (m, 4H, 2 x H), 6.69-7.18 (m, 10H, Har), 7.18-7.24 (m, 2H, HAr), 7.43 (d, $J=8.2 \mathrm{~Hz}, 2 \mathrm{H}, \mathrm{HAr}) .{ }^{13} \mathrm{C}$ NMR (125 MHz, $\left.\mathrm{CDCl}_{3}\right): \delta=21.1$, 21.2, 22.0, 22.3, 23.4, 24.3, 27.7, 28.3, 28.4, 28.8, 29.4, 29.6, 29.7, 30.7, 30.9, 31.0, 31.8, 33.8, $33.9,34.0,34.7,35.4,37.7,37.9,44.0,53.3,53.6,63.6,119.2,119.4,125.7,125.8,126.0$, 126.3, 126.5, 128.0, 128.1, 128.2, 129.1, 129.4, 134.2, 134.7, 134.8, 140.0, 142.2, 145.3, 145.4, 146.2, 156.7, 156.8, 157.9. HR-MS (ESI): $\mathrm{m} / \mathrm{z}$ calcd for $\mathrm{C}_{46} \mathrm{H}_{57} \mathrm{~N}_{12} \quad[\mathrm{M}+\mathrm{H}]^{+}:$777.4824; found: 777.4800. FT-IR (neat): $\tilde{v}=2930$ (m), 2103 (s), 1515 (w), 1450 (m), 1369 (w), 1250 (m), $1141(\mathrm{~m}), 1050(\mathrm{~m}), 912(\mathrm{~m}), 809(\mathrm{w}), 730(\mathrm{~s}), 644(\mathrm{w}), 562(\mathrm{w})$. 


\section{Compound 27}

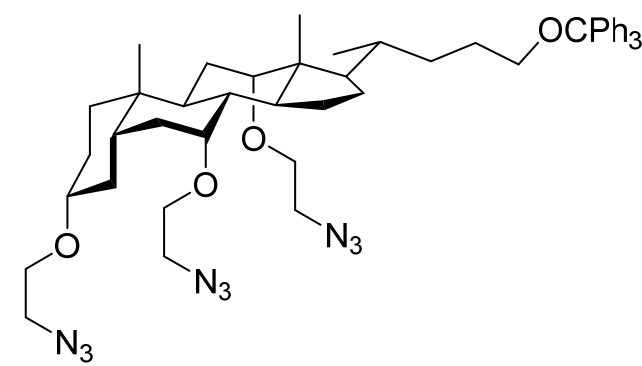

26

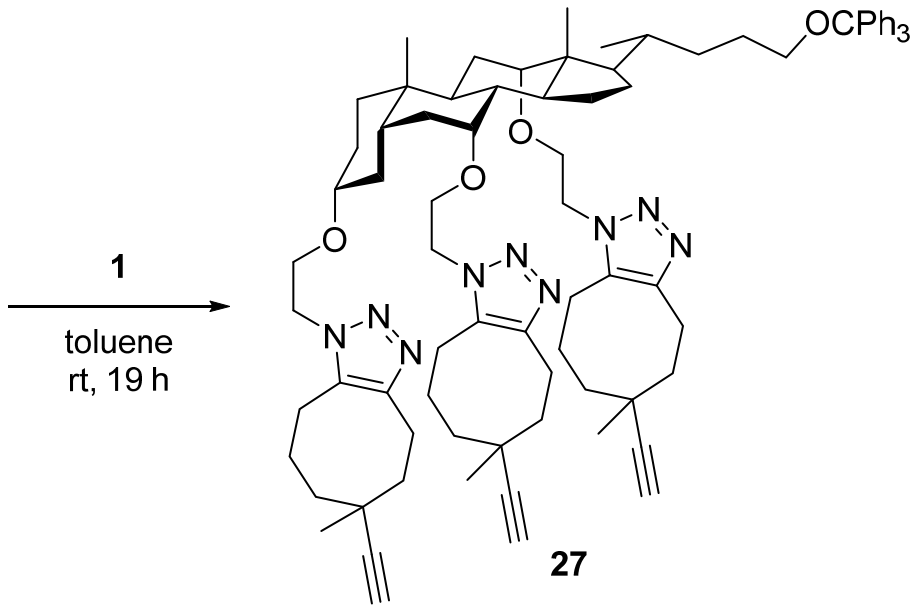

Triazide 26 (150 mg, $0.18 \mathrm{mmol}, 1.00 \mathrm{eq})$ was dissolved in toluene $(2.5 \mathrm{~mL})$ and a solution of cyclooctyne 1 (104 mg, $0.71 \mathrm{mmol}, 4.00 \mathrm{eq})$ in toluene $(0.5 \mathrm{~mL})$ was added. The resulting solution was stirred at $\mathrm{rt}$ for $19 \mathrm{~h}$. The solvent was removed under reduced pressure and the residue was purified by column chromatography on silica $\left(\mathrm{CHCl}_{3} / \mathrm{MeOH} 30: 1\right)$ to give product 27 (mixture of regioisomers and diastereomers, $227 \mathrm{mg}, 0.18 \mathrm{mmol}, 99 \%$ ) as a colorless foam. Analytical data for mixture of isomeres: m.p. $\left(\mathrm{CHCl}_{3}\right)$ : $102{ }^{\circ} \mathrm{C}$. TLC (EtOAc): $\mathrm{R}_{f}=0.32$. ${ }^{1} \mathbf{H}$ NMR (500 MHz, $\mathrm{CDCl}_{3}$ ): $\delta=0.51-2.35$ (series of multipletts, 63H), 2.76-3.31 (series of multipletts, 16H), 3.42-3.70 (m, 3H), 3.80-4.02 (m, 4H), 4.22-4.50 (m, 6H), 7.20-7.25 (m, $\left.3 \mathrm{H}, \mathrm{H}_{A r}\right), 7.27-7.33\left(\mathrm{~m}, 6 \mathrm{H}, \mathrm{H}_{A r}\right), 7.42-7.47\left(\mathrm{~m}, 6 \mathrm{H}, \mathrm{H}_{A r}\right)$. HR-MS (ESI): $\mathrm{m} / \mathrm{z}$ calcd for $\mathrm{C}_{82} \mathrm{H}_{108} \mathrm{~N}_{9} \mathrm{O}_{4}[\mathrm{M}+\mathrm{H}]^{+}:$1282.8519; found: 1282.8513. FT-IR (neat): $\widetilde{v}=3295(\mathrm{w}), 2927(\mathrm{~m})$, 2862 (m), 2020 (w), 1470 (m), 1447 (m), 1376 (w), 1106 (s), 748 (s), 706 (s), 632 (s), 552 (w), $448(\mathrm{w})$. 


\section{Compound 28}

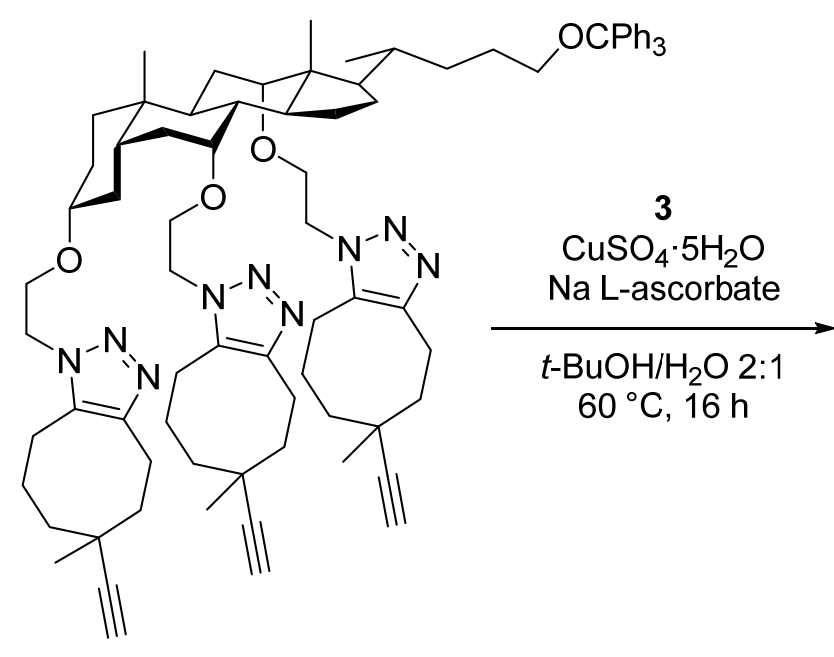

27

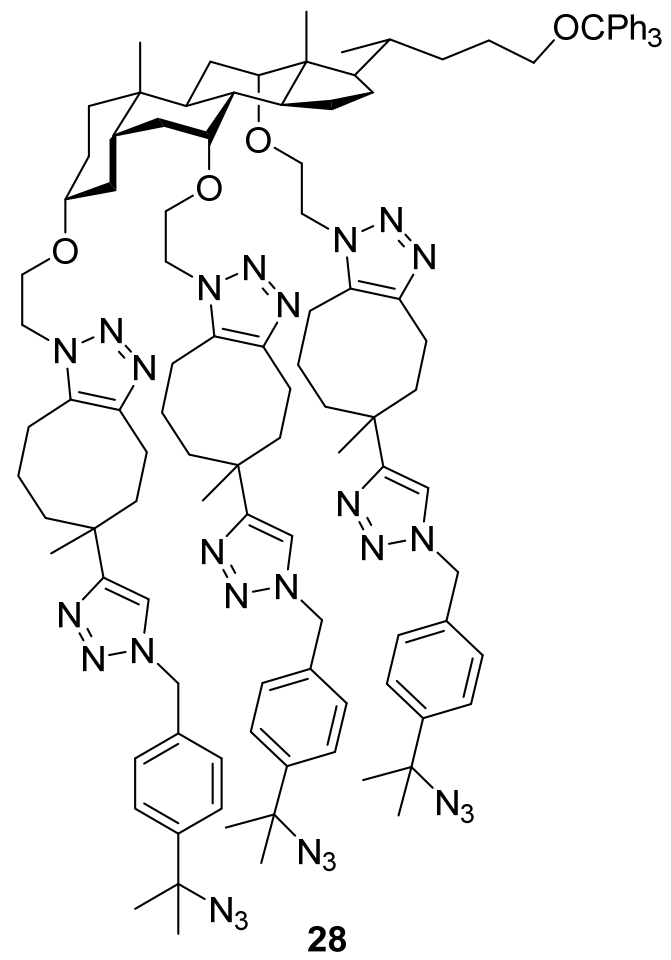

28

Compound 27 (mixture of regioisomers and diastereomers, $60 \mathrm{mg}, 0.047 \mathrm{mmol}, 1.00 \mathrm{eq}$ ) and bisazide 3 (35 mg, $0.16 \mathrm{mmol}, 3.50 \mathrm{eq})$ were dissolved in $t-\mathrm{BuOH} / \mathrm{H}_{2} \mathrm{O}(2: 1,1.5 \mathrm{~mL})$ and the solution was degassed by means of two freeze-pump-thaw cycles. Sodium L-ascorbate $(4.6 \mathrm{mg}$, $0.023 \mathrm{mmol}, 0.50 \mathrm{eq})$ and $\mathrm{CuSO}_{4} \cdot 5 \mathrm{H}_{2} \mathrm{O}(2.3 \mathrm{mg}, 0.01 \mathrm{mmol}, 0.20 \mathrm{eq})$ were added and the reaction mixture was stirred for $16 \mathrm{~h}$ at $60{ }^{\circ} \mathrm{C}$. Then $10 \%$ aq. $\mathrm{NH}_{3}(5 \mathrm{~mL})$ was added and the mixture was extracted with EtOAc $(3 \times 10 \mathrm{~mL})$. The combined organic layer was dried over $\mathrm{MgSO}_{4}$ and the solvent was removed under reduced pressure. The remaining crude product was purified by column chromatography on silica $\left(\mathrm{CHCl}_{3} / \mathrm{MeOH} 20: 1\right)$ to give compound 28 (mixture of regioisomers and diastereomers, $91 \mathrm{mg}, 0.047 \mathrm{mmol}$, quant.) as a colorless foam. Analytical data for mixture of isomeres: TLC $\left(\mathrm{CHCl}_{3} / \mathrm{MeOH} 20: 1\right): \mathrm{R}_{f}=0.22 .{ }^{1} \mathbf{H}$ NMR (600 MHz, $\mathrm{CD}_{2} \mathrm{Cl}_{2}$ ): $\delta=0.51-2.69$ (series of multipletts, $78 \mathrm{H}$ ), 2.73-3.29 (series of multipletts, 16H), 3.40-3.68 (m, 3H), 3.70-4.02 (m, 4H),4.10-4.46 (m, 6H), 5.34-5.51 (m, 6H, HBenzyl), 7.17-7.32 (m, 16H, $\left.\mathrm{H}_{A r}\right)$, 7.36-7.48 (m, 14H, $\left.\mathrm{H}_{A r}\right)$. HR-MS (ESI): $\mathrm{m} / \mathrm{z}$ calcd for $\mathrm{C}_{112} \mathrm{H}_{143} \mathrm{~N}_{27} \mathrm{O} 4 \mathrm{Na}[\mathrm{M}+\mathrm{Na}]^{+}:$1954.1738; found: 1954.1712. FT-IR (neat): $\widetilde{v}=2931(\mathrm{~m})$, 2862 (m), 2100 (s), 1447 (m), 1367 (m), 1248 (m), 1097 (s), 1049 (s), 813 (m), 748 (s), 706 (s), $631(\mathrm{~m}), 561(\mathrm{w}), 476(\mathrm{w}), 426(\mathrm{w})$. 


\section{Compound 29}
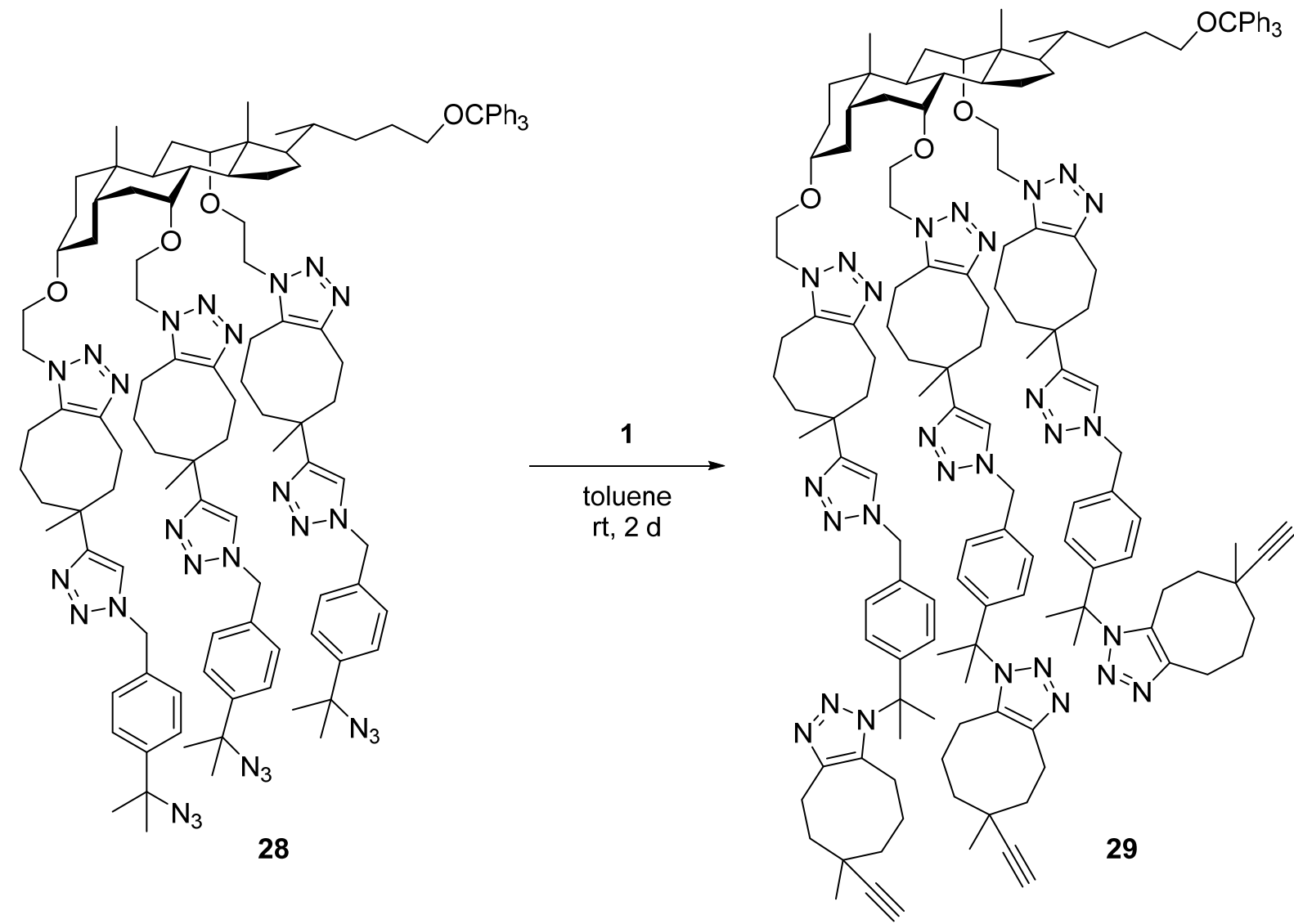

Triazide 28 (mixture of regioisomers and diastereomers, $31 \mathrm{mg}, 0.016 \mathrm{mmol}, 1.00 \mathrm{eq}$ ) was dissolved in toluene $(0.5 \mathrm{~mL})$ and a solution of cyclooctyne 1 (10 mg, $0.068 \mathrm{mmol}, 4.25 \mathrm{eq})$ in toluene $(0.5 \mathrm{~mL})$ was added. The resulting solution was stirred for $2 \mathrm{~d}$ at $\mathrm{rt}$. Then the solvent was removed under reduced pressure. The residue was purified by column chromatography on silica $\left(\mathrm{CHCl}_{3} / \mathrm{MeOH} 40: 1\right.$ to $\left.30: 1\right)$ to give product 29 (mixture of regioisomers and diastereomers, $33 \mathrm{mg}, 0.014 \mathrm{mmol}, 86 \%)$ as a colorless gum. TLC $\left(\mathrm{CHCl}_{3} / \mathrm{MeOH}\right.$ 20:1): $\mathrm{R}_{f}=0.15 .{ }^{1} \mathbf{H}$ NMR $\left(500 \mathrm{MHz}, \mathrm{CDCl}_{3}\right): \delta=0.51-2.74$ (series of multipletts, $\left.114 \mathrm{H}\right), 2.76-3.30$ (m, 22H), 3.39-3.67 (m, 3H), 3.70-4.01 (m, 4H), 4.13-4.48 (m, 6H), 5.33-5.52 (m, 6H, HBenzyl), 7.00-7.48 (m, 30H, $\left.\mathrm{H}_{A r}\right) .{ }^{13} \mathbf{C}$ NMR (125 MHz, $\left.\mathrm{CDCl}_{3}\right): \delta=12.2,19.9,21.3,21.4,21.8,21.9$, $22.4,22.7,23.1,23.7,23.8,23.9,28.7,29.6,29.8,29.9,30.4,30.8,34.5,34.8,34.9,36.2,37.8$, $39.3,39.5,41.8,47.9,63.9,64.1,64.3,68.9,69.8,76.9,86.3,90.2,90.8,119.8,120.0,125.8$, $126.9,127.8,127.9,127.9,128.3,128.7,131.3,133.4,134.9,144.1,144.7,146.0$, 146.9. HRMS (ESI): $m / z$ calcd for $\mathrm{C}_{145} \mathrm{H}_{185} \mathrm{~N}_{27} \mathrm{O}_{4} \mathrm{Na}_{2}[\mathrm{M}+2 \mathrm{Na}]^{2+}$ : 1207.7461; found: 1207.7446. FT-IR (neat): $\widetilde{v}=2964(\mathrm{~m}), 1594(\mathrm{~m}), 1577(\mathrm{w}), 1497(\mathrm{~m}), 1396(\mathrm{~m}), 1325(\mathrm{~m}), 1264$ (s), 1185 (s), $1145(\mathrm{~s}), 1087(\mathrm{~m}), 981(\mathrm{w}), 837(\mathrm{w}), 766(\mathrm{w}), 671(\mathrm{w})$. 


\section{Compound 30}

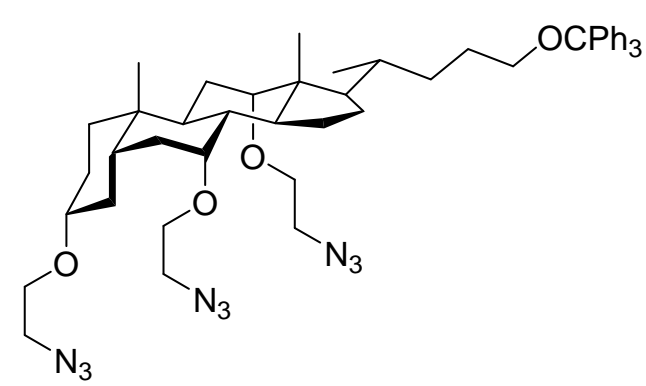

26

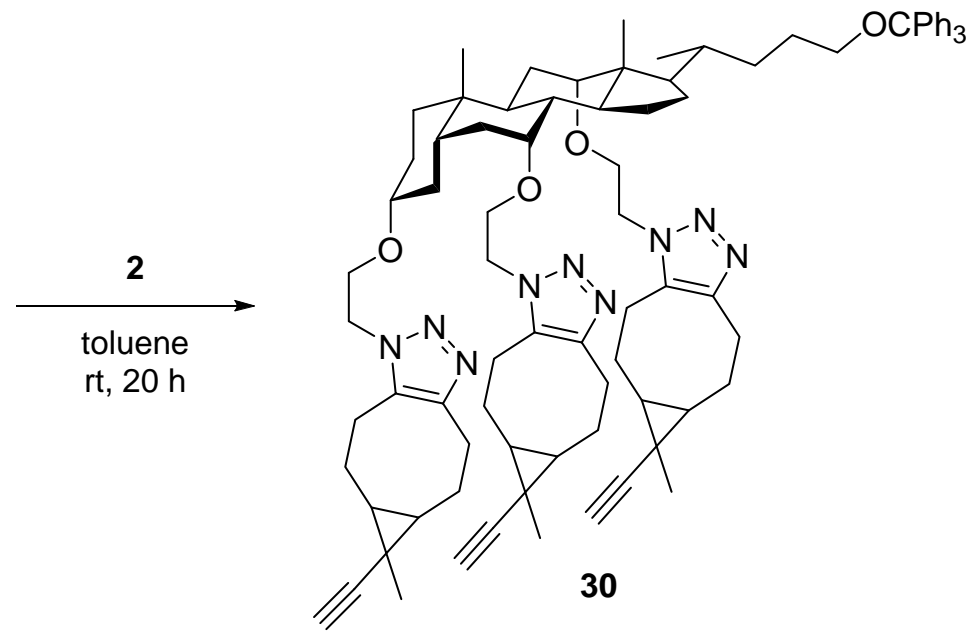

Triazide 26 (37.0 mg, $0.044 \mathrm{mmol}, 1.00 \mathrm{eq})$ was dissolved in toluene $(2.5 \mathrm{~mL})$ and a solution of cyclooctyne 2 (41.6 mg, $0.263 \mathrm{mmol}, 6.00 \mathrm{eq})$ in toluene $(0.5 \mathrm{~mL})$ was added. The resulting solution was stirred at $\mathrm{rt}$ for $20 \mathrm{~h}$. The solvent was removed under reduced pressure and the residue was purified by column chromatography on silica $\left(\mathrm{CHCl}_{3}: \mathrm{MeOH} ; 30: 1\right)$ to give 30 (57.0 $\mathrm{mg}, 0.432 \mathrm{mmol}, 99 \%)$ as a colorless foam.

TLC (EtOAc): $\mathrm{R}_{f}=0.11 .{ }^{1} \mathbf{H}$ NMR (500 MHz, $\left.\mathrm{CDCl}_{3}\right): \delta=7.45-7.43(\mathrm{~m}, 6 \mathrm{H}, \mathrm{H}$ Ar), 7.31-7.28 (m, $\left.6 \mathrm{H}, \mathrm{H}_{A r}\right), 7.24-7.21$ (m, $\left.3 \mathrm{H}, \mathrm{H}_{A r}\right), 4.44-4.33$ (m, $\left.6 \mathrm{H}\right), 3.97-3.81(\mathrm{~m}, 4 \mathrm{H}), 3.63-3.45$ (m, $2 \mathrm{H}), 3.24-3.22(\mathrm{~m}, 1 \mathrm{H}), 3.15-2.73$ (series of multipletts, $14 \mathrm{H}), 2.32-0.51$ (series of multipletts, $65 \mathrm{H}) .$. m.p.: $105{ }^{\circ} \mathrm{C}\left(\mathrm{CDCl}_{3}\right) .{ }^{13} \mathrm{C}$ NMR $\left(125 \mathrm{MHz}, \mathrm{CDCl}_{3}\right): \delta=12.4,12.4,12.4,12.5,12.5$, $12.7,12.7,12.9,13.6,13.6,13.6,13.7,13.7,13.7,13.7,17.5,20.8,21.9,22.2,22.2,22.6,22.6$, 22.6, 22.7, 22.7, 22.8, 22.9, 25.0, 25.2, 25.3, 27.2, 27.7, 28.2, 28.2, 28.5, 28.6, 29.3, 29.3, 29.4, 29.5, 29.6, 29.7, 29.7, 32.3, 34.8, 34.8, 34.9, 35.5, 35.6, 39.4, 41.6, 42.7, 42.7, 46.2, 46.2, 46.3, 47.0, 47.0, 47.8, 48.0, 48.2, 62.5, 62.8, 62.8, 62.8, 63.9, 63.9, 66.9, 67.2, 86.3, 92.3, 92.3, 126.8, 126.8, 127.7, 128.2, 128.7, 133.1, 143.9,144.0, 144.5, 144.5, 144.5. HR-MS (ESI): $\mathrm{m} / \mathrm{z}$ calcd for $\mathrm{C}_{85} \mathrm{H}_{108} \mathrm{~N}_{9} \mathrm{O}_{4}$ : $1318.8530[\mathrm{M}+\mathrm{H}]^{+}$; found: 1318.8519. FT-IR (neat): $\tilde{v}=3290(\mathrm{w})$, 2922 (s), 2854 (w), 2105 (w), 1447 (m), 1378 (w), 1312 (w), 1220 (w), $1155(\mathrm{w}), 1106$ (s), $975(\mathrm{w}), 923(\mathrm{w}), 801(\mathrm{w}), 749(\mathrm{~s}), 705$ (m), 632 (m), 601 (w), $519(\mathrm{w}), 498(\mathrm{w})$. 


\section{Compound 31}

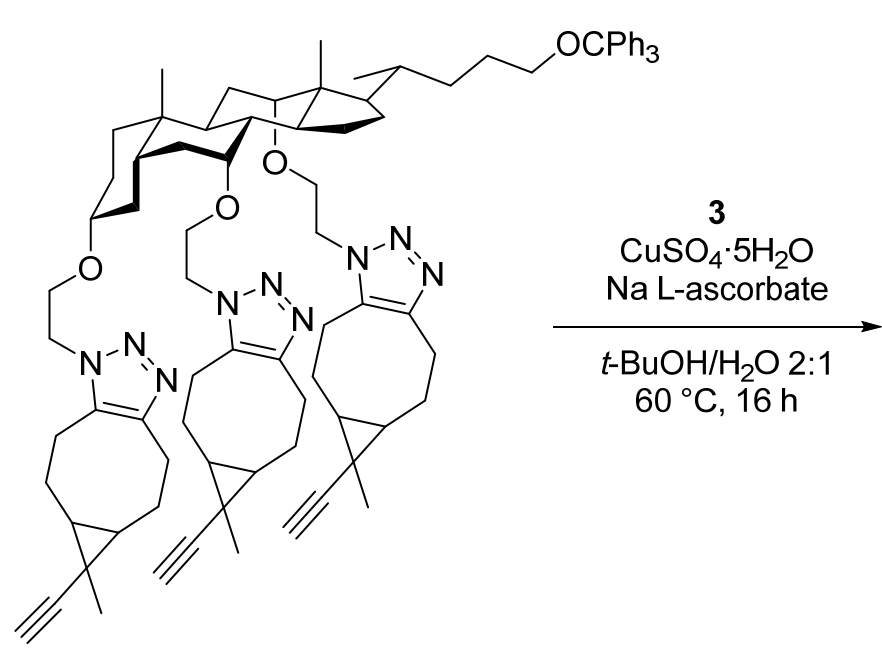

30

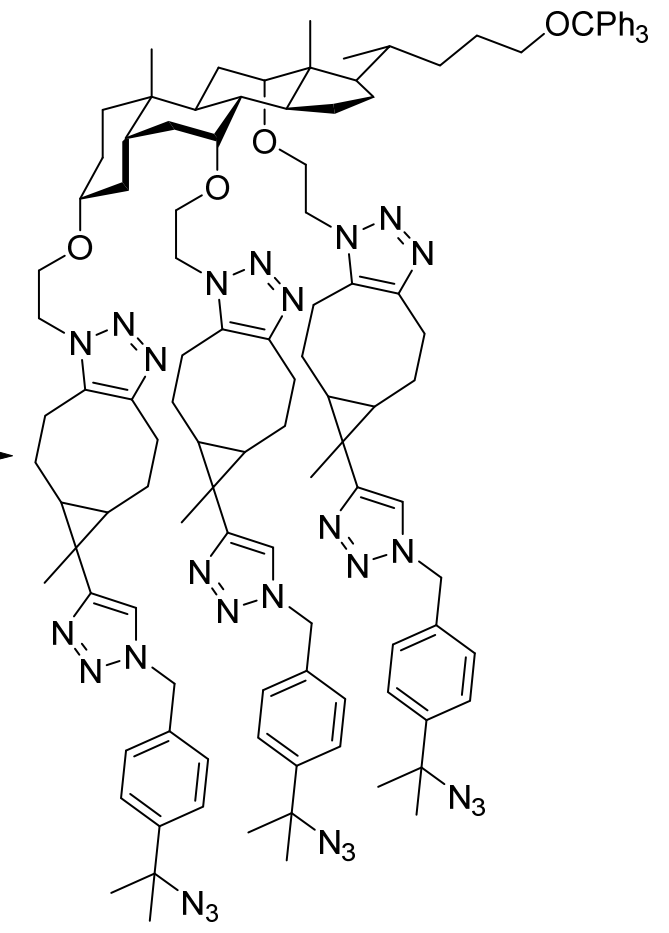

31

Compound 30 (mixture of regioisomers and diastereomers, $40.0 \mathrm{mg}, 0.030 \mathrm{mmol}, 1.00 \mathrm{eq}$ ) and bisazide 3 (23.0 mg, $0.106 \mathrm{mmol}, 3.50 \mathrm{eq})$ were dissolved in $t-\mathrm{BuOH} / \mathrm{H}_{2} \mathrm{O}(2: 1,1.5 \mathrm{~mL})$ and the solution was degassed by means of two freeze-pump-thaw cycles. Sodium L-ascorbate ( $3.1 \mathrm{mg}, 0.015 \mathrm{mmol}, 0.50 \mathrm{eq})$ and $\mathrm{CuSO}_{4} \cdot 5 \mathrm{H}_{2} \mathrm{O}(1.5 \mathrm{mg}, 0.006 \mathrm{mmol}, 0.20 \mathrm{eq})$ were added and the reaction mixture was stirred for $20 \mathrm{~h}$ at $60{ }^{\circ} \mathrm{C}$. Then $10 \%$ aq. $\mathrm{NH}_{3}(5.0 \mathrm{~mL})$ was added and the mixture was extracted with EtOAc $(3 \times 10 \mathrm{~mL})$. The combined organic layer was dried over $\mathrm{MgSO}_{4}$ and the solvent was removed under reduced pressure. The remaining crude product was purified by column chromatography on silica $\left(\mathrm{CHCl}_{3} / \mathrm{MeOH} 20: 1\right)$ to give compound 31 (mixture of regioisomers and diastereomers, $59 \mathrm{mg}, 0.029 \mathrm{mmol}, 98 \%$ ) as a colorless foam.

TLC $\left(\mathrm{CHCl}_{3}: \mathrm{MeOH} 20: 1\right): \mathrm{R}_{f}=0.15 .{ }^{1} \mathbf{H}$ NMR $\left(500 \mathrm{MHz}, \mathrm{CD}_{2} \mathrm{Cl}_{2}\right): \delta=7.45-7.43(\mathrm{~m}, 11 \mathrm{H}$, $\mathrm{H}_{A r}$ ), 7.30-7.20 (m, $\left.16 \mathrm{H}, \mathrm{H}_{A r}\right)$, 5.43-5.32 (m, $\left.6 \mathrm{H}\right), 4.35-4.32(\mathrm{~m}, 6 \mathrm{H}), 3.83-0.57$ (series of multipletts, $104 \mathrm{H})$. m.p.: $97{ }^{\circ} \mathrm{C}\left(\mathrm{CHCl}_{3}\right)$. HR-MS (ESI): $\mathrm{m} / \mathrm{z}$ calcd for $\mathrm{C}_{115} \mathrm{H}_{144} \mathrm{~N}_{27} \mathrm{O}_{4}$ : 1967.1899 $[\mathrm{M}+\mathrm{H}]^{+}$; found: $1967.1889 .{ }^{13} \mathrm{C} \mathbf{N M R}\left(125 \mathrm{MHz}, \mathrm{CDCl}_{3}\right): \delta=12.1,12.2,12.3$, $12.4,12.4,12.5,17.3,22.5,22.6,22.8,22.9,22.9,23.0,23.0,23.1,23.3,25.3,25.5,28.1,28.9$, 29.4, 29.7, 29.7, 30.0, 34.7, 34.9, 35.5, 39.3, 41.7, 46.1, 46.3, 48.2, 63.6, 64.0, 64.1, 77.6, 86.2, 118.9, 119.0, 119.0, 125.8, 125.8, 125.8, 126.8, 127.1, 127.7, 127.9, 127.9, 127.9, 128.0, 128.1, 128.1, 128.6, 134.6, 134.6, 144.6, 145.0, 145.1, 145.2, 155.5, 155.5, 155.6. FT-IR (neat): $\tilde{v}=2926(w), 2862(w), 2101(m), 1550(w), 1513(w), 1447(w), 1366(w), 1312(w)$, 
$1254(w), 1216(w), 1186(w), 1150(w), 1099(m), 1048(w), 1019(w), 994(w), 922(w)$, $813(w), 749(s), 706(w), 664(w), 647(w), 629(w), 562(w), 500(w), 476(w), 423(w)$.

\section{Compound 32}
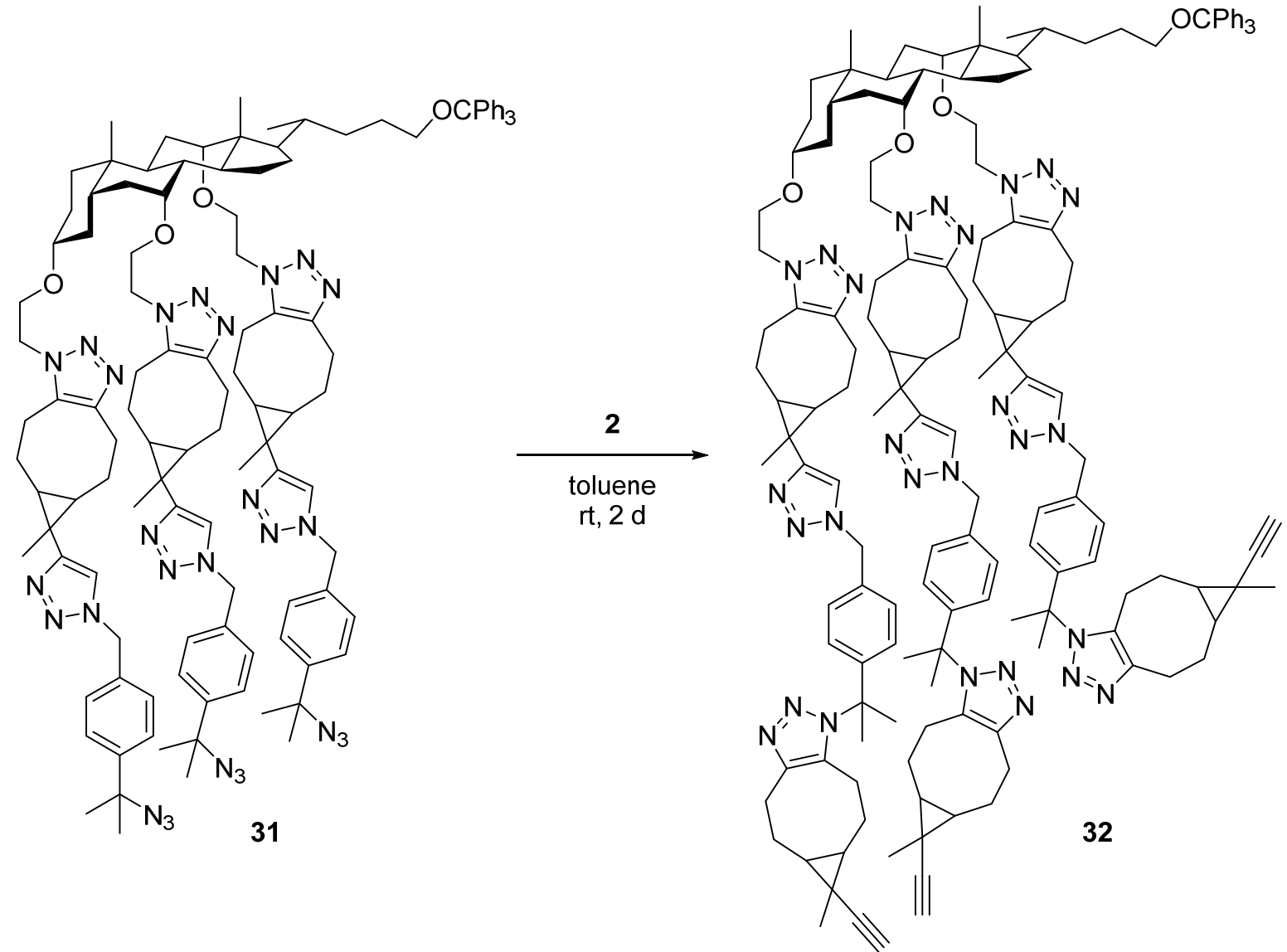

Triazide 31 (mixture of regioisomers and diastereomers, $34.0 \mathrm{mg}, 0.017 \mathrm{mmol}, 1.00 \mathrm{eq}$ ) was dissolved in toluene $(2.5 \mathrm{~mL})$ and a solution of cyclooctyne 2 (16.4 mg, $0.104 \mathrm{mmol}, 6.00 \mathrm{eq})$ in toluene $(0.5 \mathrm{~mL})$ was added. The resulting solution was stirred for $2 \mathrm{~d}$ at $\mathrm{rt}$. Then the solvent was removed under reduced pressure. The residue was purified by column chromatography on silica $\left(\mathrm{CHCl}_{3} / \mathrm{MeOH} 40: 1\right.$ to $\left.30: 1\right)$ to give product 32 (mixture of regioisomers and diastereomers, $41.4 \mathrm{mg}, 0.017 \mathrm{mmol}, 98 \%)$ as a colorless solid. TLC $\left(\mathrm{CHCl}_{3} / \mathrm{MeOH}\right.$ 20:1): $\mathrm{R}_{f}=0.11 .{ }^{1} \mathbf{H}$ NMR $\left(300 \mathrm{MHz}, \mathrm{CD}_{2} \mathrm{Cl}_{2}\right): \delta=7.45-7.37\left(\mathrm{~m}, 10 \mathrm{H}, \mathrm{H}_{A r}\right), 7.31-7.11(\mathrm{~m}, 17 \mathrm{H}$, $\mathrm{H}_{\mathrm{Ar}}$ ), 5.48-5.36 (m, $\left.6 \mathrm{H}\right)$, 4.47-4.27 (m, $\left.6 \mathrm{H}\right), 4.00-3.74$ (m, $\left.4 \mathrm{H}\right), 3.63-3.44$ (m, $\left.3 \mathrm{H}\right), 3.28-2.75$ $(\mathrm{m}, 19 \mathrm{H}), 2.52-0.49$ (series of multipletts, $125 \mathrm{H}) .{ }^{13} \mathbf{C}$ NMR $\left(125 \mathrm{MHz}, \mathrm{CDCl}_{3}\right): \delta=12.1$, $12.2,12.3,12.4,12.4,12.5,13.9,17.0,17.2,17.3,18.8,18.9,18.9,19.0,19.1,19.2,19.3,19.7$, $19.7,21.9,22.0,22.3,22.5,22.5,22.6,22.8,22.9,22.9,23.0,23.0,23.1,23.2,23.3,23.4,23.4$, 25.3, 25.5, 25.6, 26.9, 27.0, 27.4, 27.5, 27.6, 28.1, 28.3, 28.4, 28.4, 28.9, 28.9, 29.0, 29.0, 29.1, 29.2, 29.3, 29.4, 29.6, 29.7, 29.7, 29.8, 29.8, 30.0, 30.0, 30.7, 31.9, 32.3, 34.7, 34.9, 35.5, 35.5, 
39.3, 41.7, 46.1, 46.2, 46.3, 46.8, 46.9, 47.0, 47.8, 48.0, 48.2, 63.6, 64.0, 64.1, 66.8, 66.9, 67.1, $67.4,67.6,67.8,70.5,76.9,77.1,77.6,80.1,80.2,80.3,81.8,82.0,86.2,118.8,118.9,119.0$, 119.0, 125.6, 125.8, 125.8, 125.8, 126.8, 127.1, 127.7, 127.9, 127.9, 127.9, 128.0, 128.1, 128.1, 128.4, 128.6, 133.2, 133.3, 133.5, 133.6, 133.6, 133.7, 134.0, 134.3, 134.6, 134.6, 134.7, 134.9, 143.4, 143.4, 143.5, 143.7, 144.0, 144.0, 144.1, 144.3, 144.3, 144.5, 144.6, 144.7, 144.9, 145.0, 145.1, 145.2, 155.5, 155.5, 155.6. HR-MS (ESI): $\mathrm{m} / \mathrm{z}$ calcd for $\mathrm{C}_{151} \mathrm{H}_{187} \mathrm{~N}_{27} \mathrm{O}_{4}: 1222.2654$ $[\mathrm{M}+2 \mathrm{H}]^{+}$; found: 1222.2632. FT-IR (neat): $\widetilde{v}=2920(\mathrm{~s}), 2851(\mathrm{~m}), 2100(\mathrm{w}), 1549(\mathrm{w})$, $1460(w), 1367(w), 1309(w), 1255(w), 1151(w), 1101(m), 1047(w), 1019(w), 923(w)$, $801(w), 767(w), 705(m), 630(w), 564(w), 498(w), 471(w)$. 
${ }^{1} \mathrm{H} \&{ }^{13} \mathrm{C}$ NMR spectra

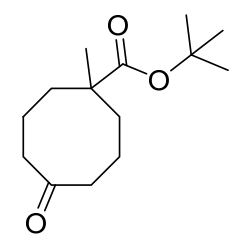

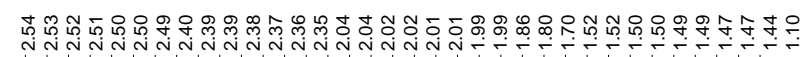

7

$500 \mathrm{MHz}, \mathrm{CDCl}_{3}$

Wh Mult

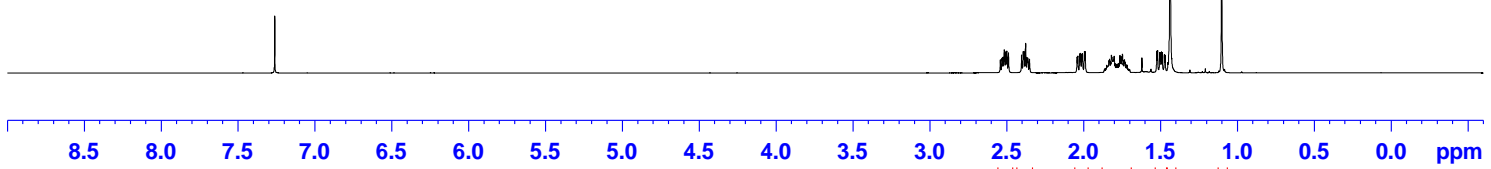

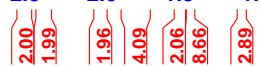
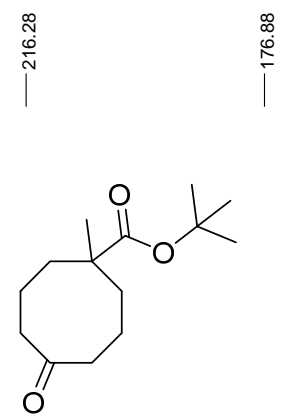

7

$125 \mathrm{MHz}, \mathrm{CDCl}_{3}$ 


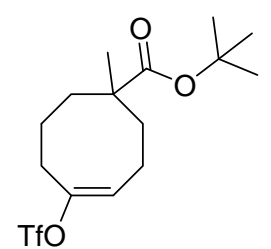

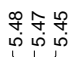

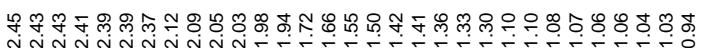

TfO

$400 \mathrm{MHz}, \mathrm{C}_{6} \mathrm{D}_{6}$
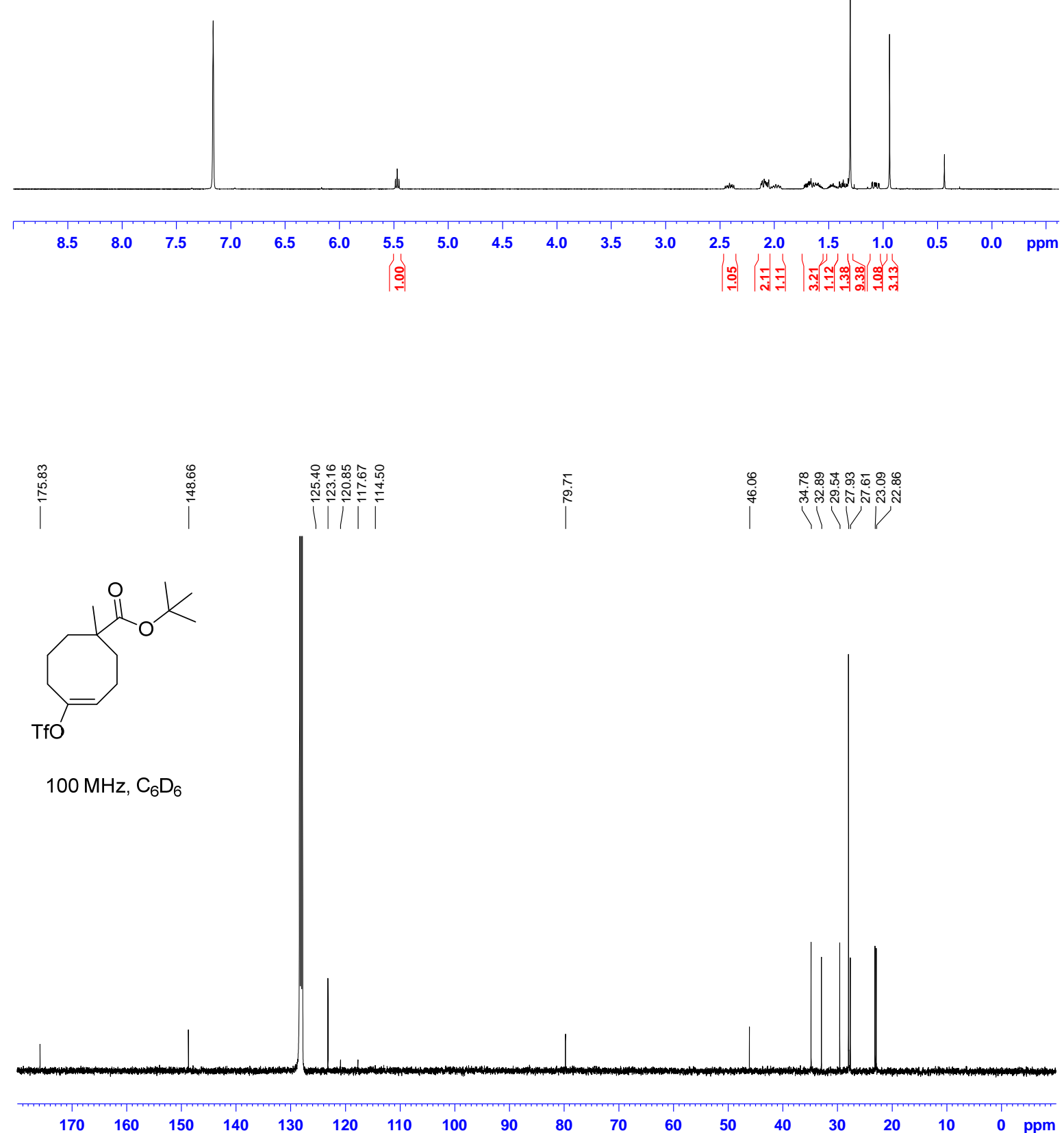


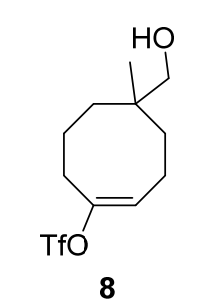

$500 \mathrm{MHz}$, acetone- $d_{6}$
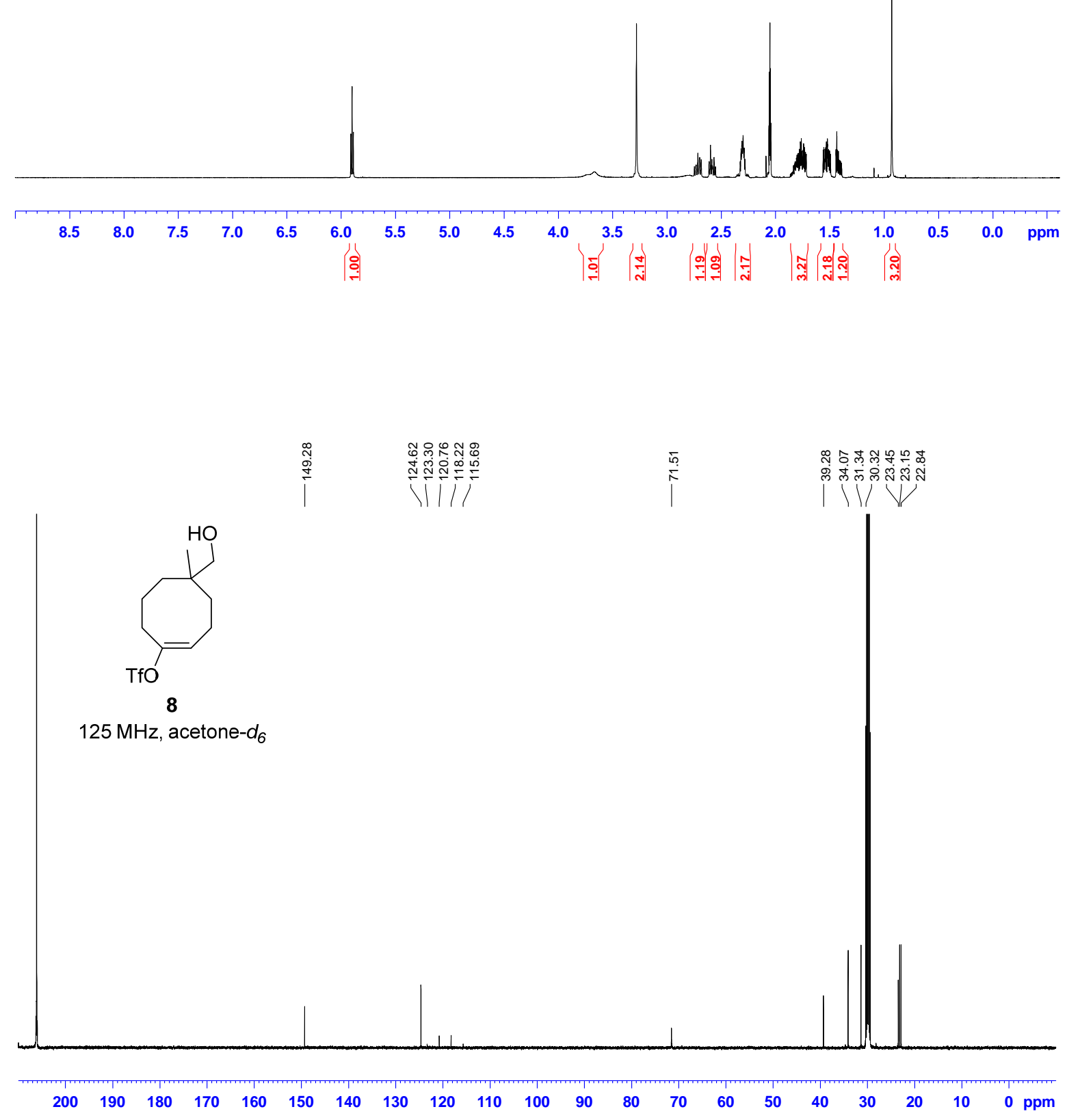


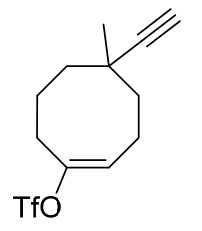

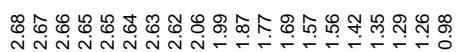

9

$500 \mathrm{MHz}, \mathrm{C}_{6} \mathrm{D}_{6}$
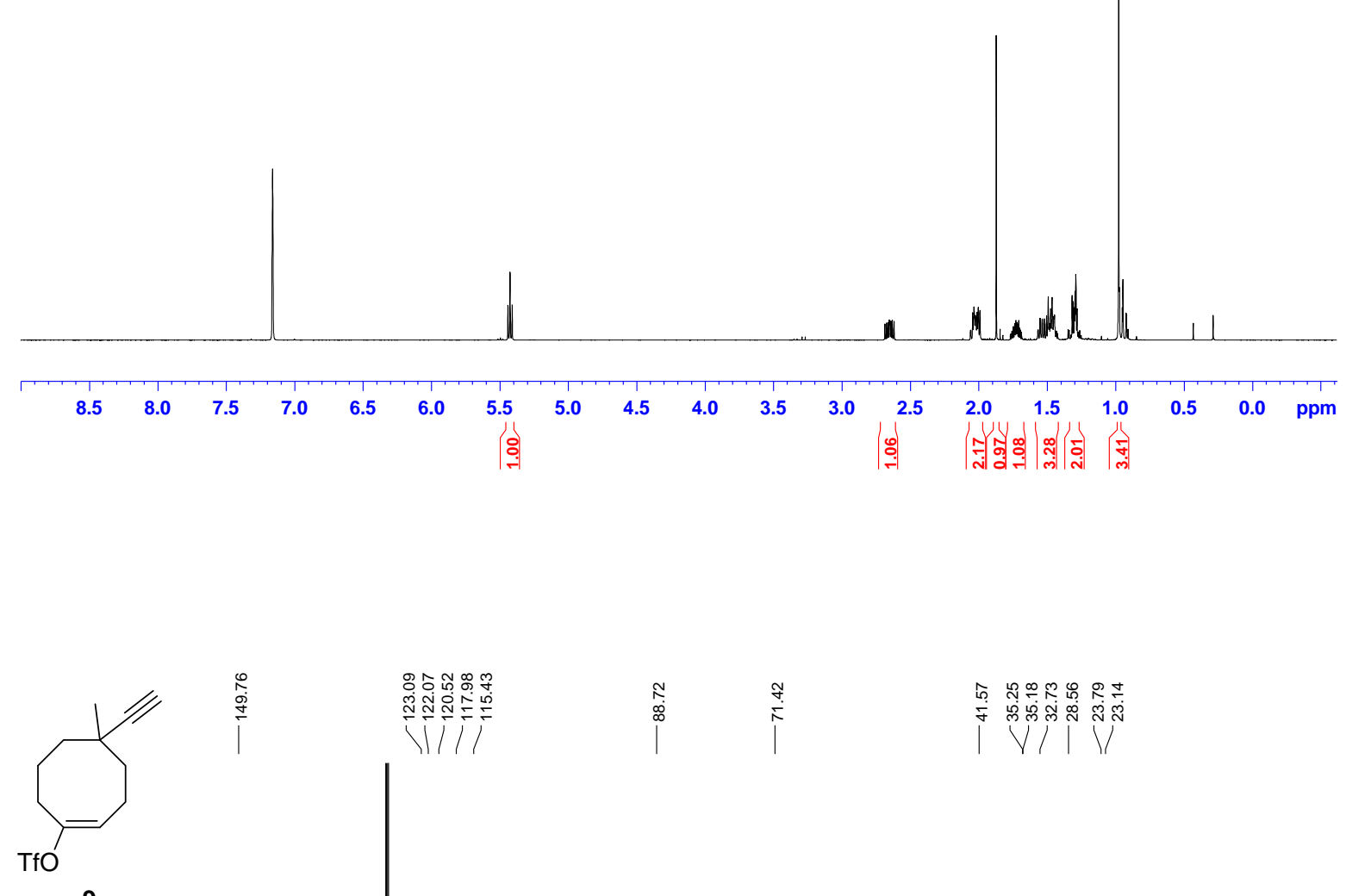

$125 \mathrm{MHz}, \mathrm{C}_{6} \mathrm{D}_{6}$

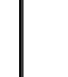

(25 $\mathrm{MHz} \mathrm{C}_{6} \mathrm{D}_{6}$

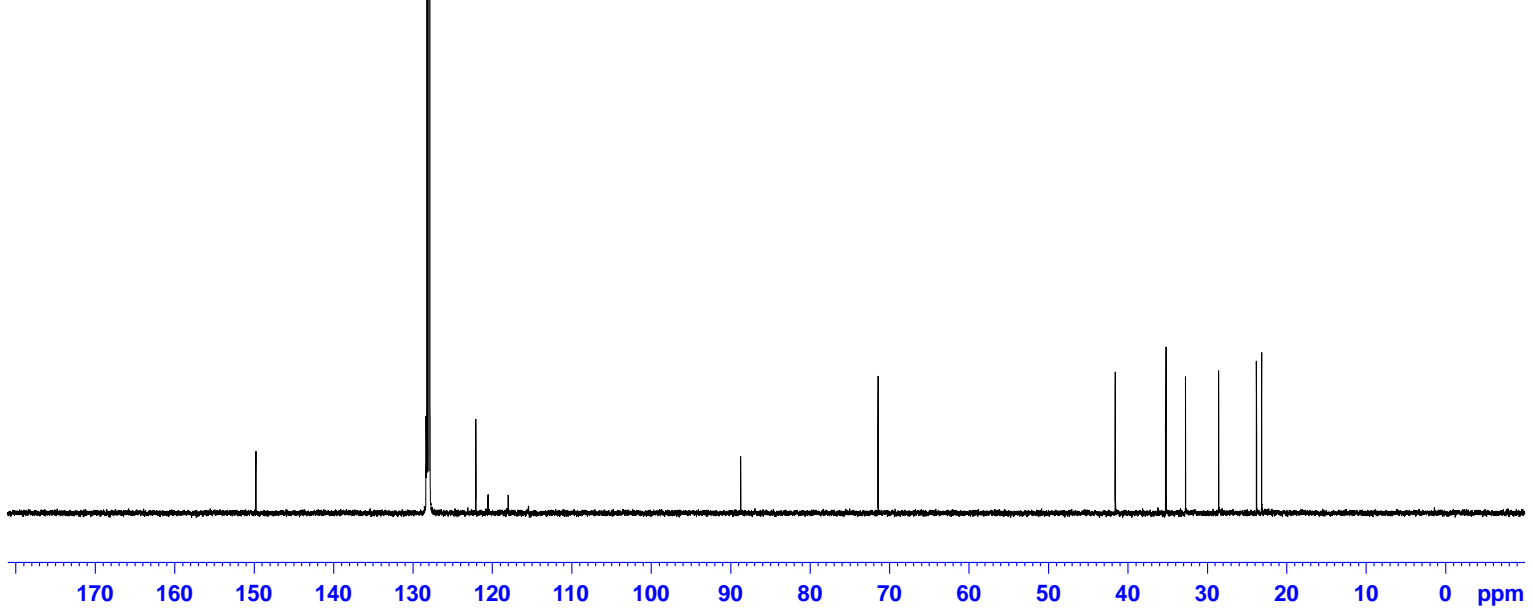




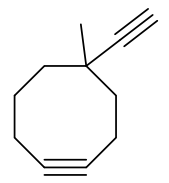

1

$500 \mathrm{MHz}, \mathrm{C}_{6} \mathrm{D}_{6}$

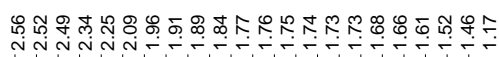

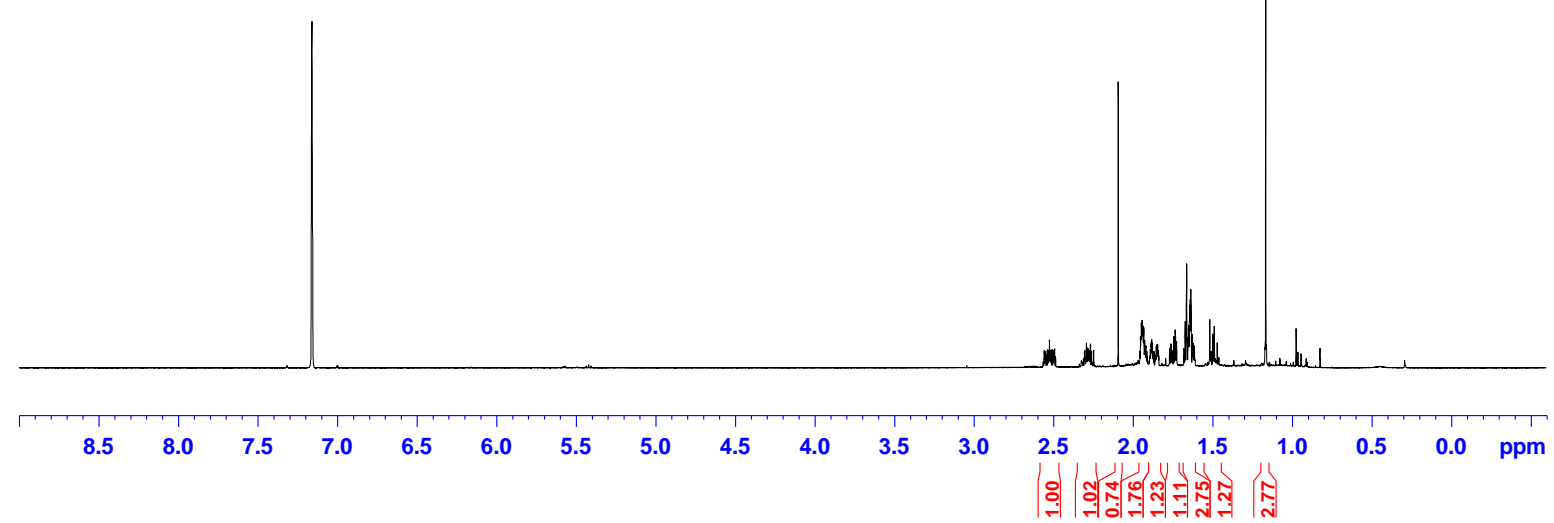

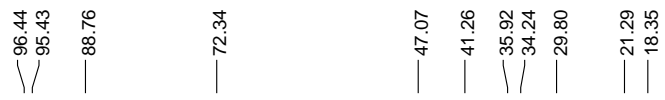

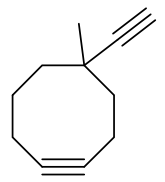

1

$125 \mathrm{MHz}, \mathrm{C}_{6} \mathrm{D}_{6}$

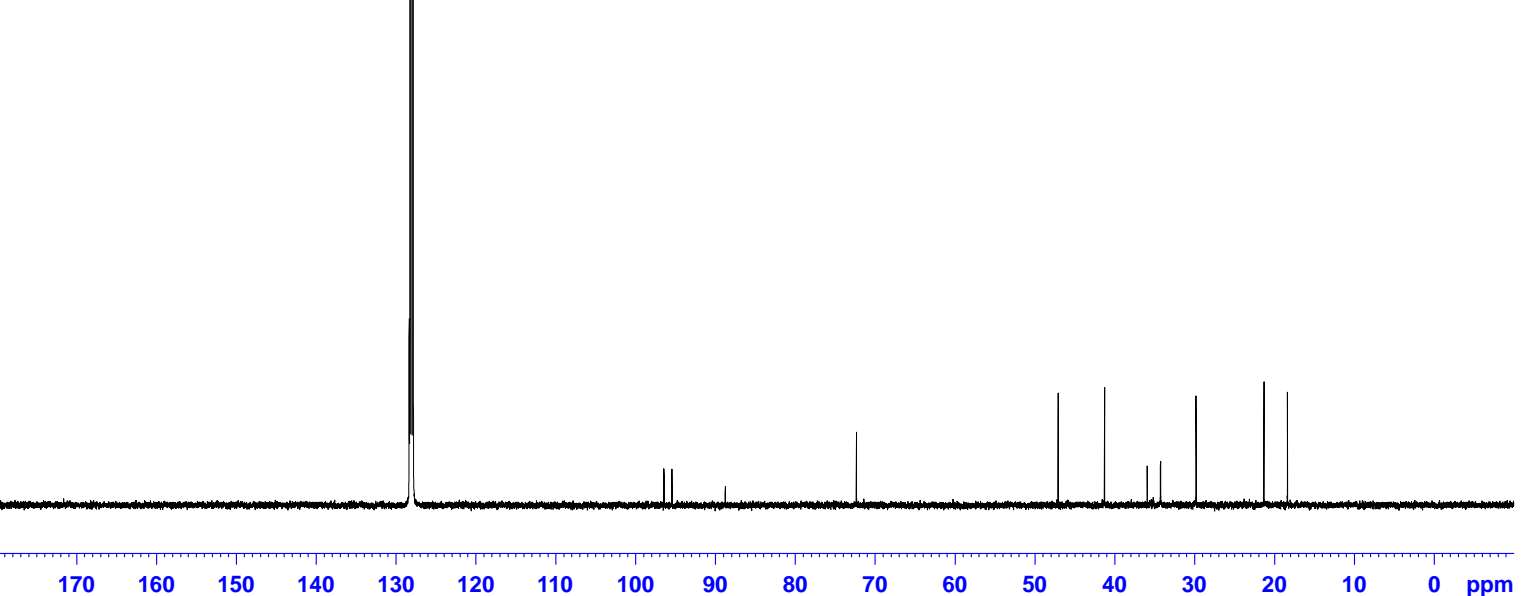


<smiles>CC1(C(=O)O)CCC=CCCC1</smiles>

S1

$500 \mathrm{MHz}, \mathrm{CDCl}_{3}$

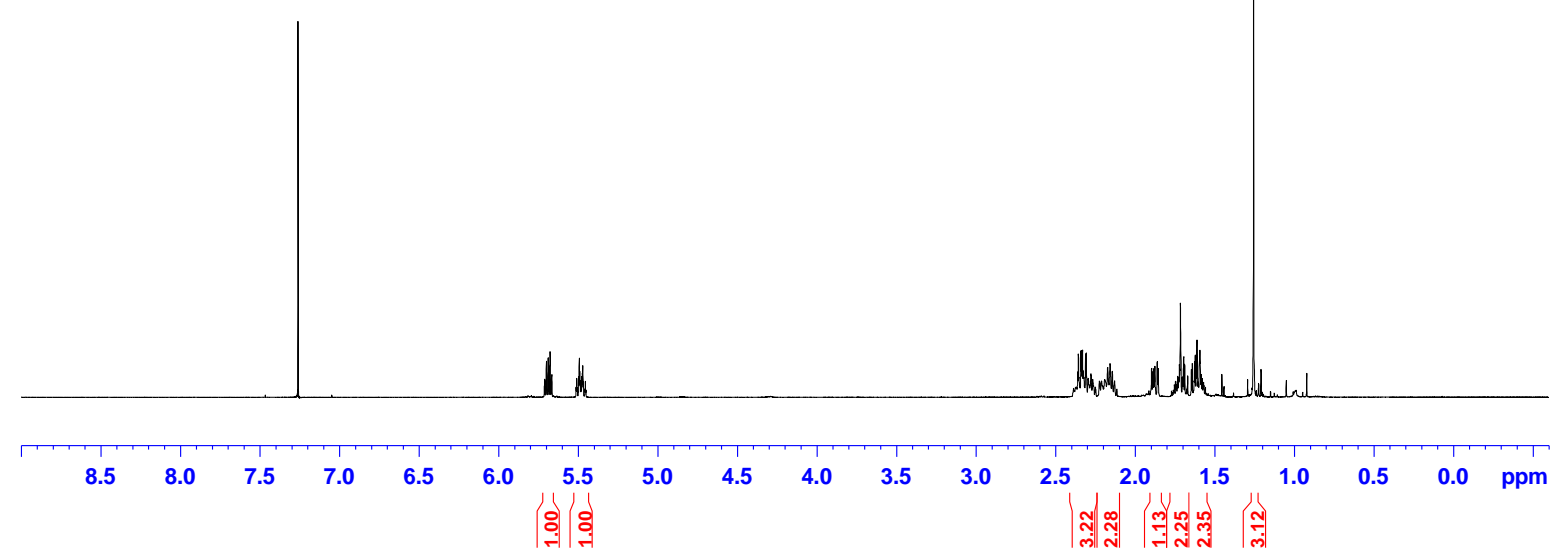

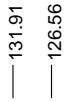

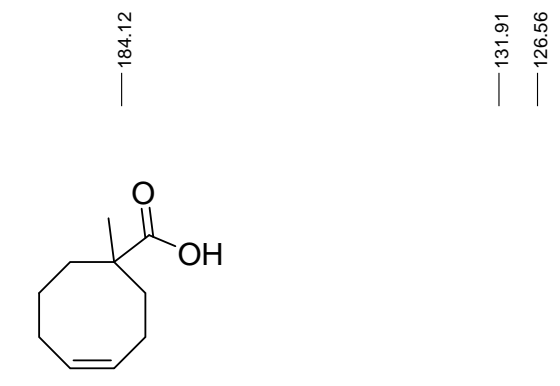

S1

$125 \mathrm{MHz}, \mathrm{CDCl}_{3}$

|

HVK

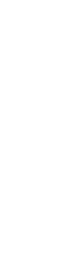


婴贶

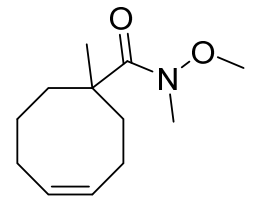

S2

$400 \mathrm{MHz}, \mathrm{CDCl}_{3}$
H. h

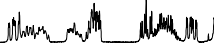
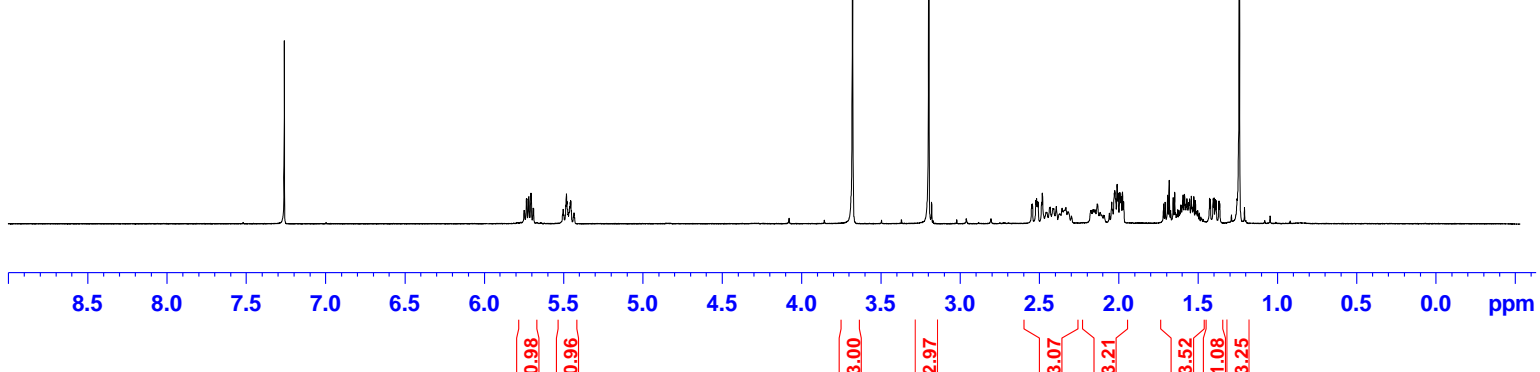

<smiles>CON(C)C(=O)C1(C)CCC=CCCC1</smiles>

S2

$100 \mathrm{MHz}, \mathrm{CDCl}_{3}$

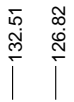

$\left.\right|^{7}$

$\mid$ 


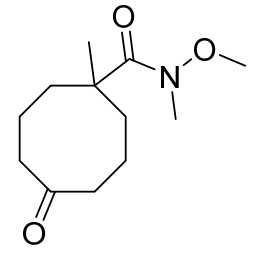

S3

$300 \mathrm{MHz}, \mathrm{CDCl}_{3}$

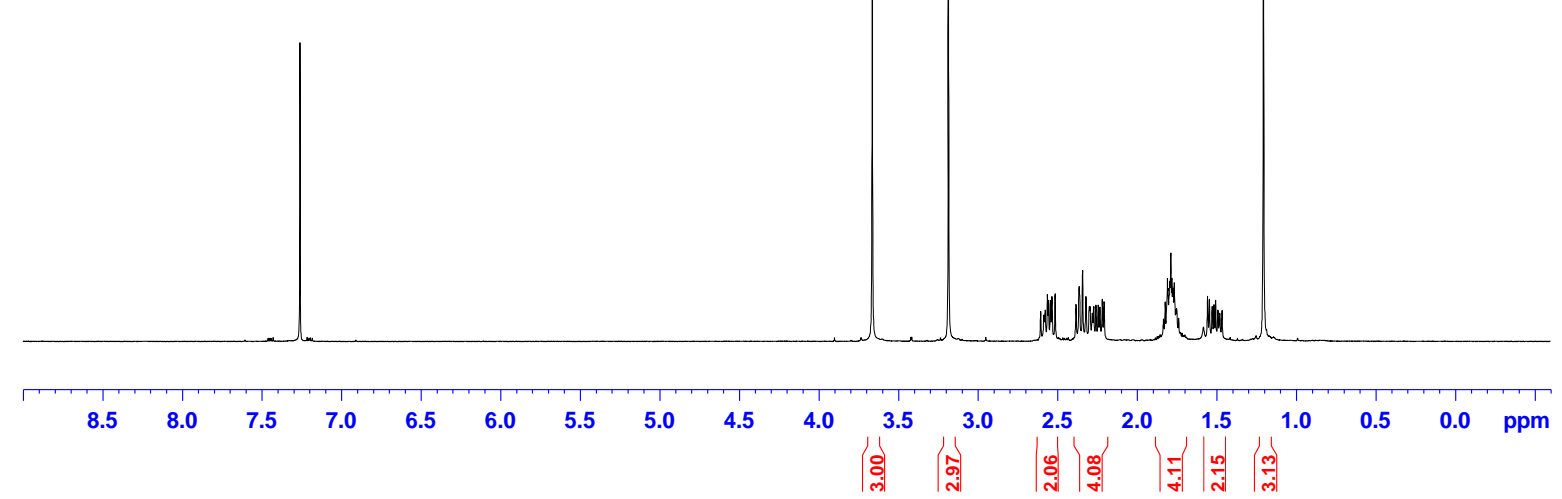

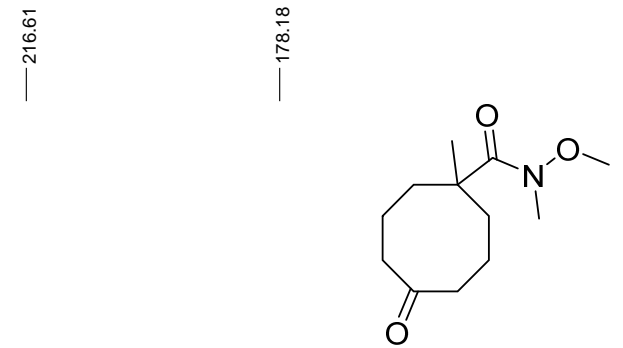

S3

$75 \mathrm{MHz} \mathrm{CDCl}_{3}$ 

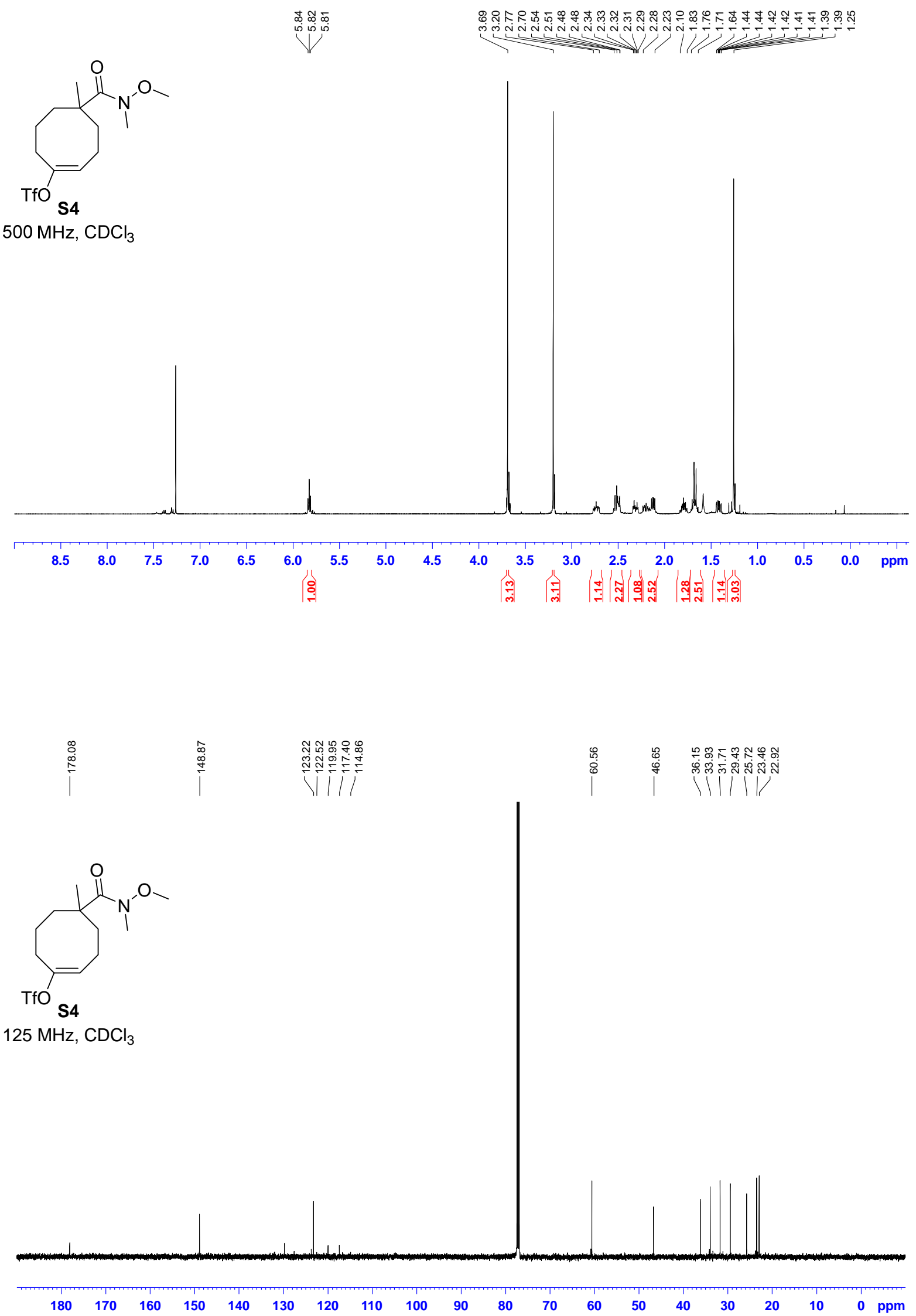


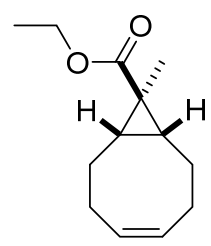

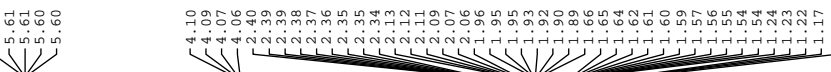

exo-11

$500 \mathrm{MHz}, \mathrm{CDCl}_{3}$

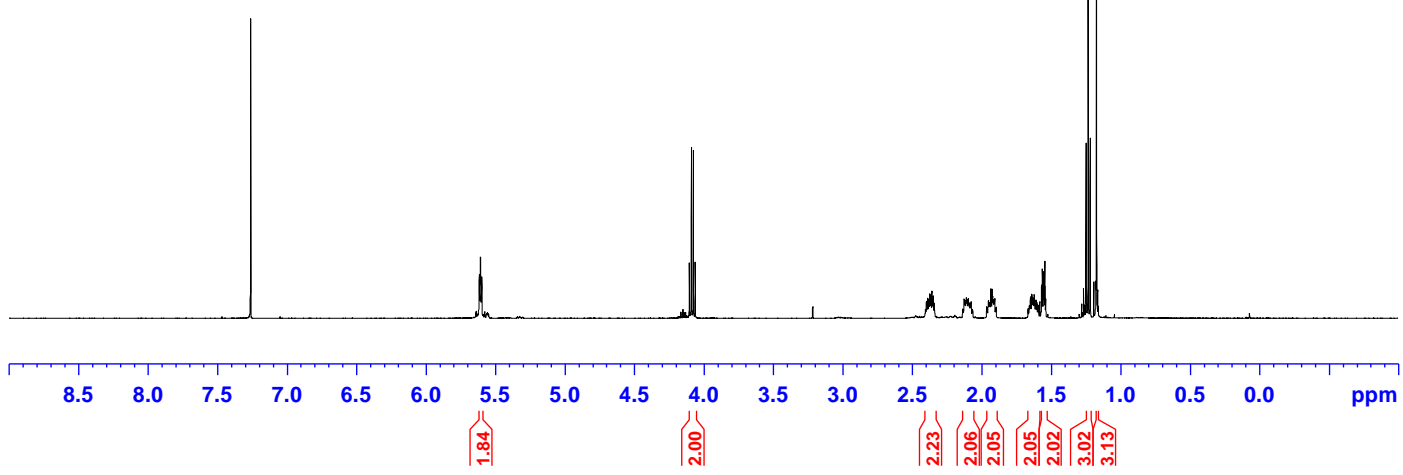

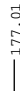
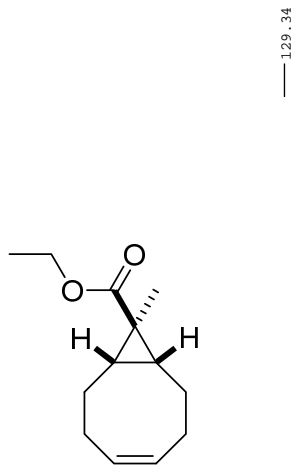

exo-11

$125 \mathrm{MHz}^{\mathrm{CDCl}} \mathrm{Cl}_{3}$
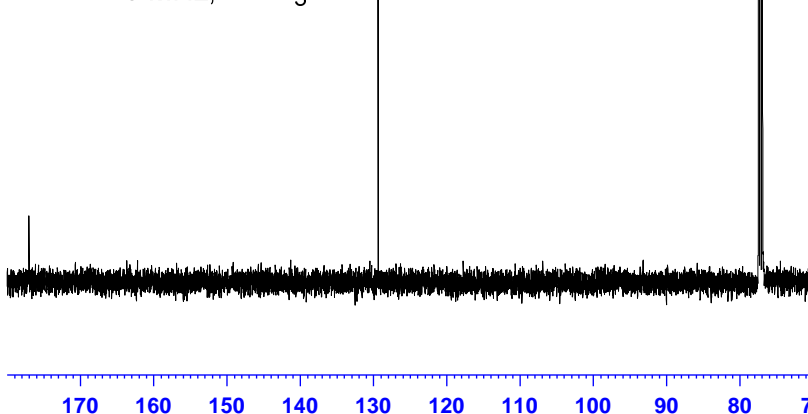

60

50

40

2

$100 \mathrm{ppm}$ 


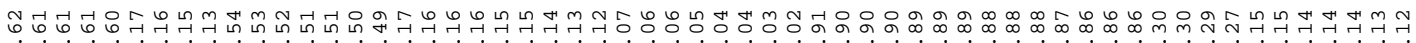
⿰纟⿴囗十)

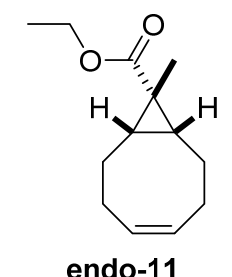

$500 \mathrm{MHz}, \mathrm{CDCl}_{3}$

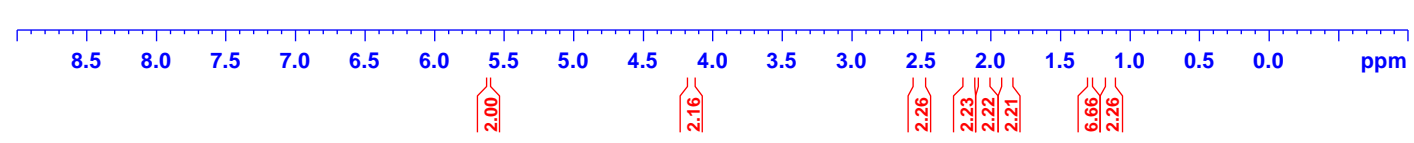

|

象

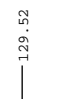

$\left.\right|^{\circ}$

$\mid$

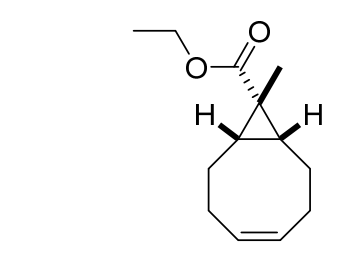

endo-11

$125 \mathrm{MHz}, \mathrm{CDCl}_{3}$

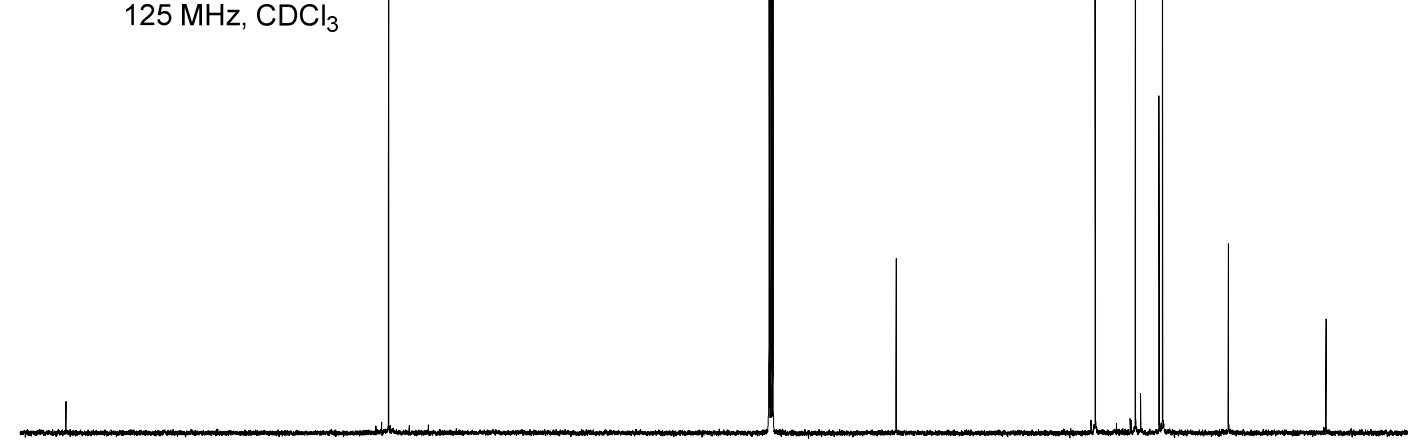

$\begin{array}{lllllllllllllllllll}170 & 160 & 150 & 140 & 130 & 120 & 110 & 100 & 90 & 80 & 70 & 60 & 50 & 40 & 30 & 20 & 10 & 0 & \mathrm{ppm}\end{array}$ 


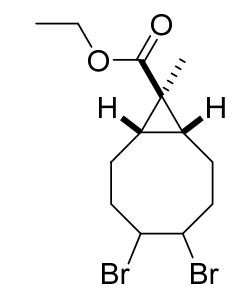

$500 \mathrm{MHz}, \mathrm{CDCl}_{3}$

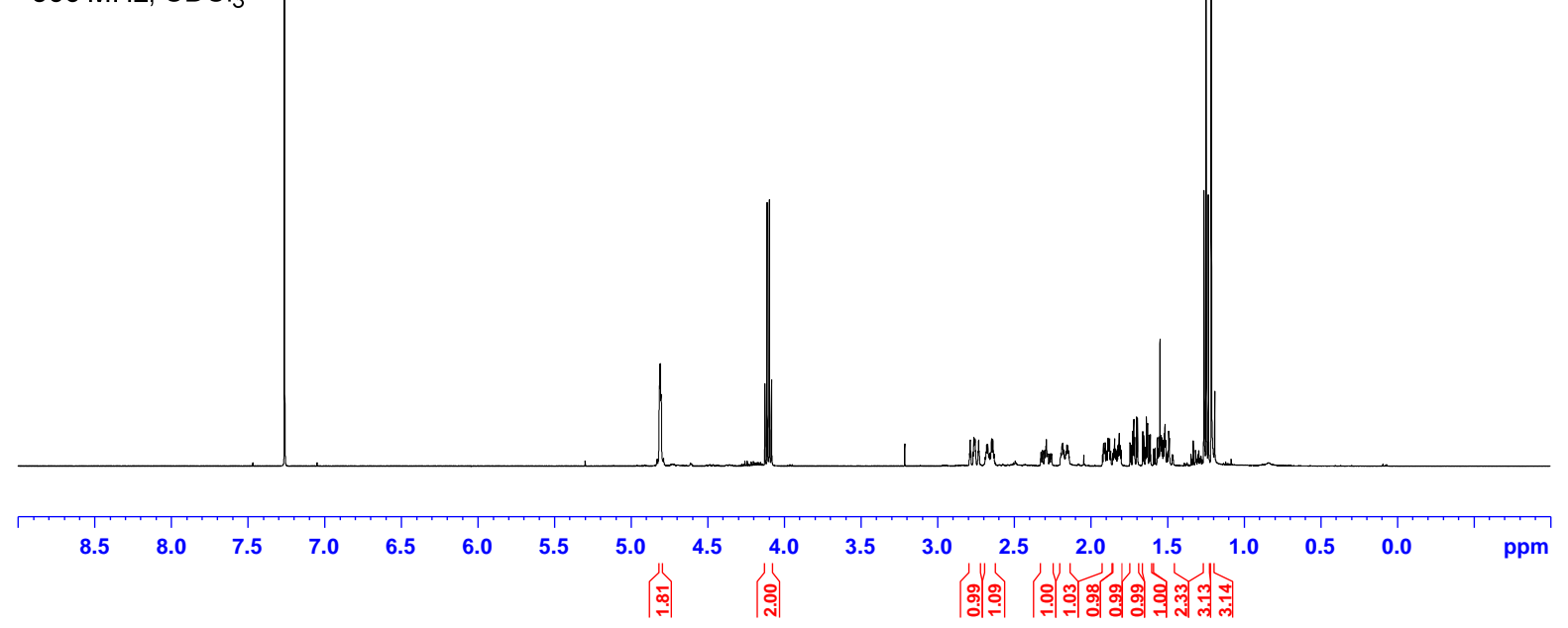

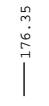

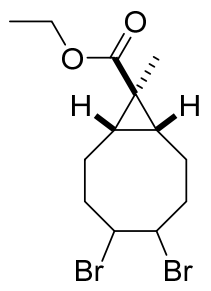

$125 \mathrm{MHz} \mathrm{CDCl}_{3}$

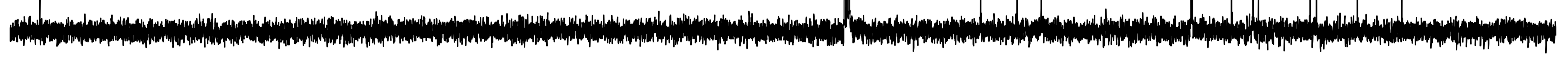
$170 \quad 160$

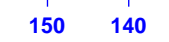
130
$120 \quad 110 \quad 100$
$90 \quad 80$
$70 \quad 60$
50
30
10
0 ppm 


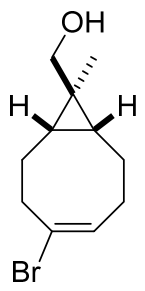

$500 \mathrm{MHz}, \mathrm{CDCl}_{3}$
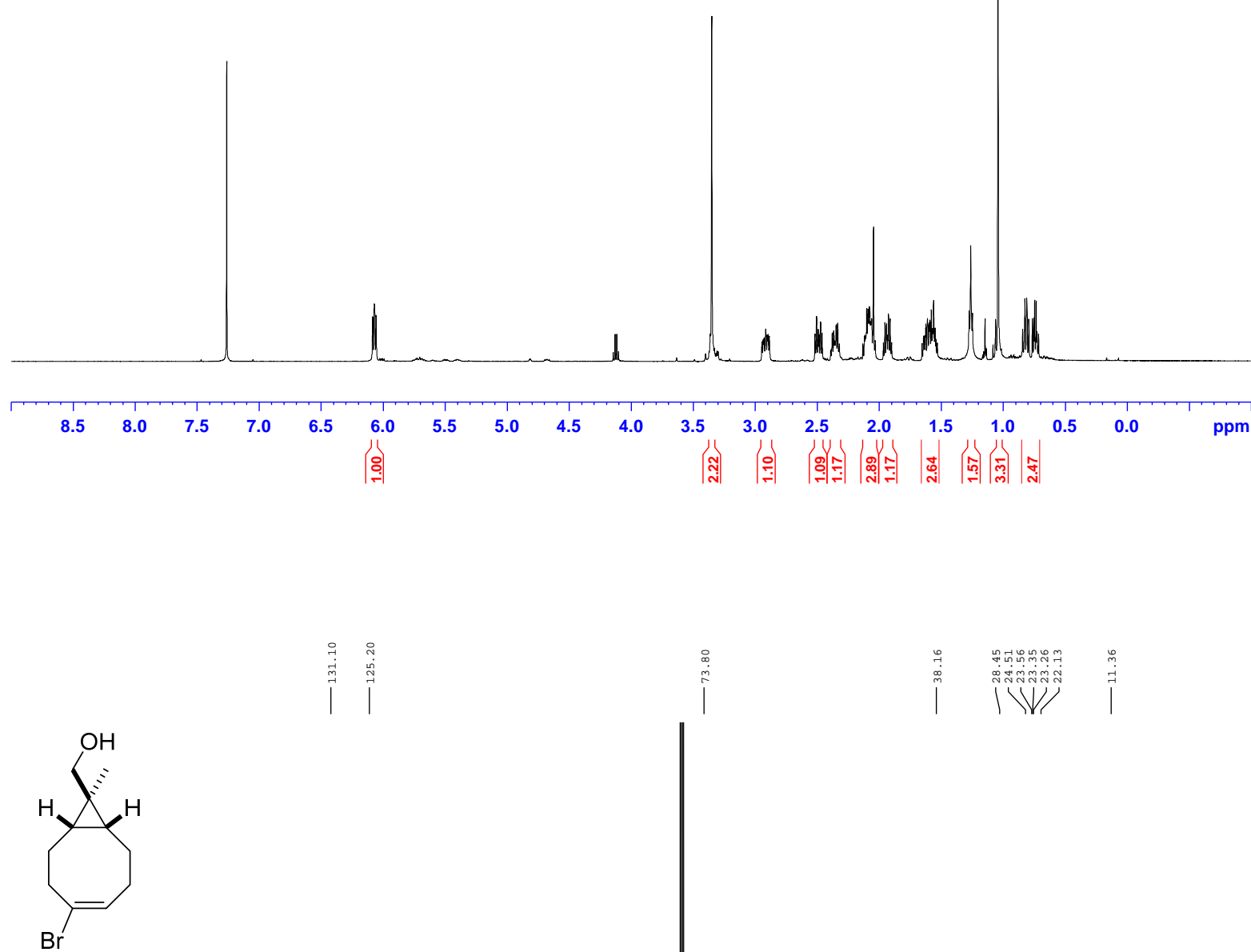

$125 \mathrm{MHz}, \mathrm{CDCl}_{3}$

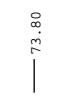

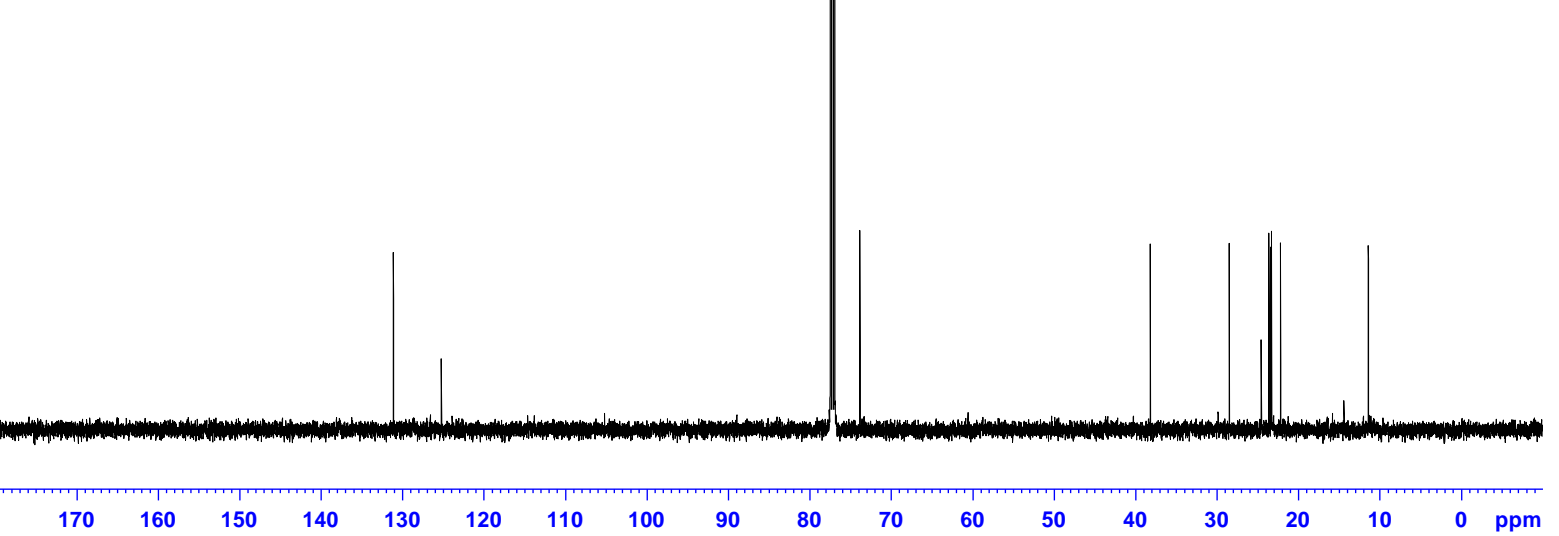




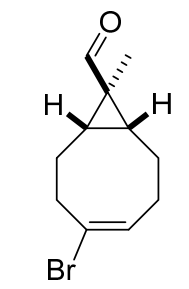

$500 \mathrm{MHz}, \mathrm{CDCl}_{3}$
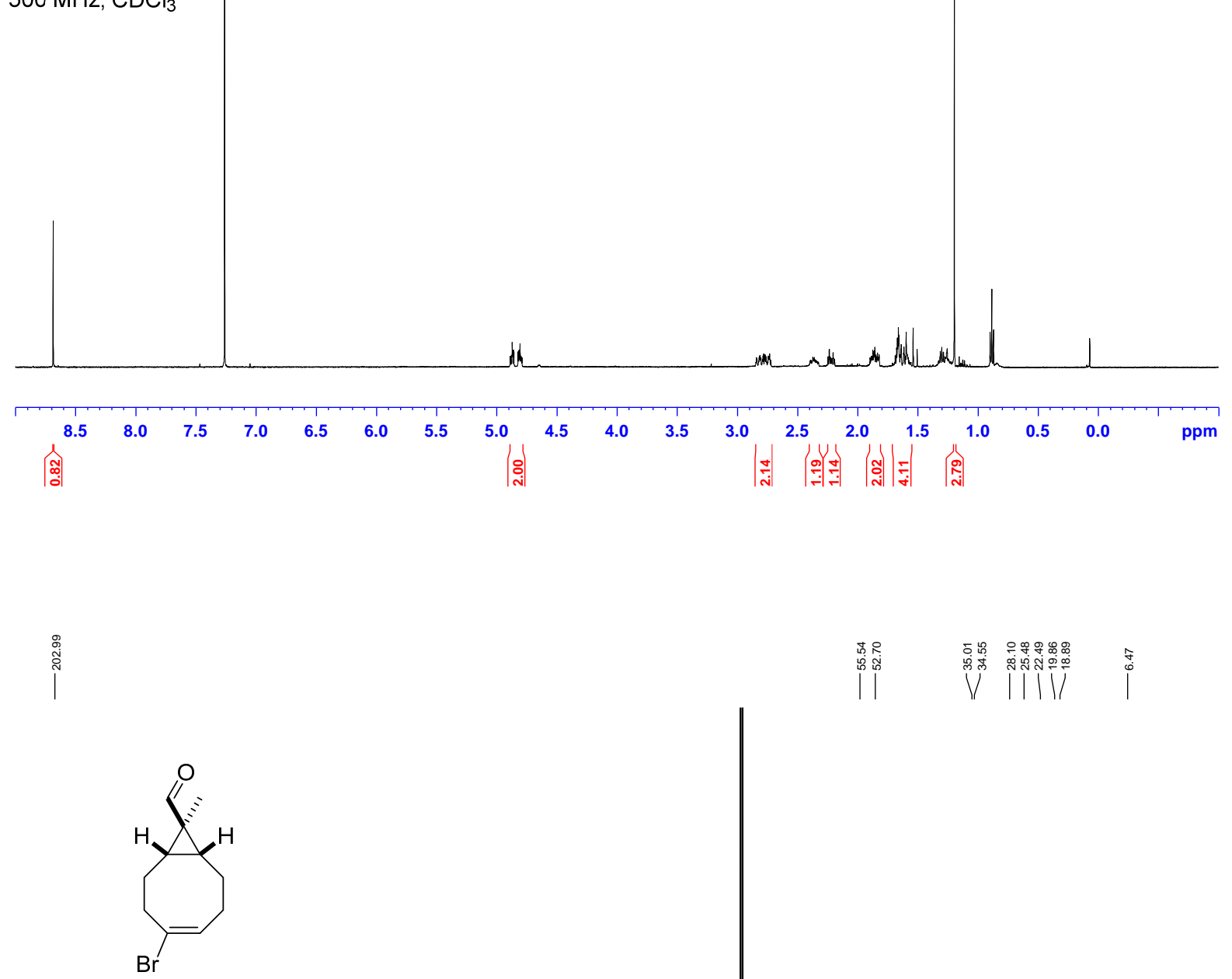

$125 \mathrm{MHz}, \mathrm{CDCl}_{3}$

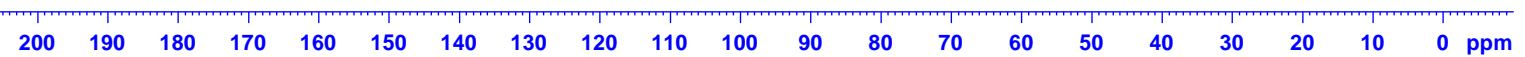




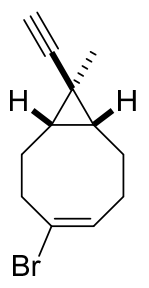

13

$500 \mathrm{MHz}, \mathrm{CDCl}_{3}$

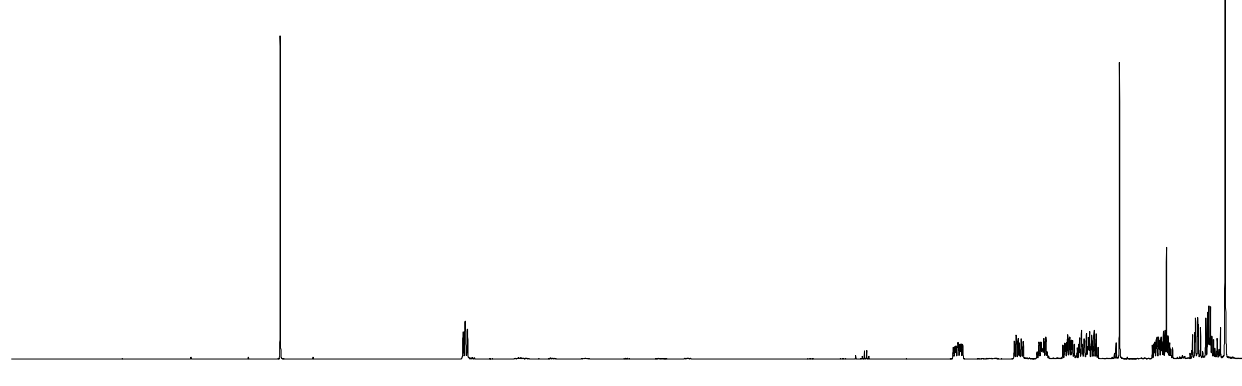

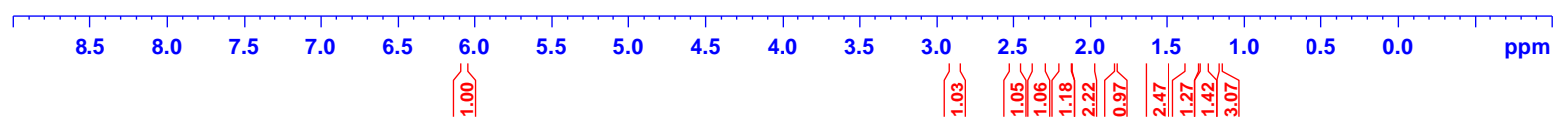
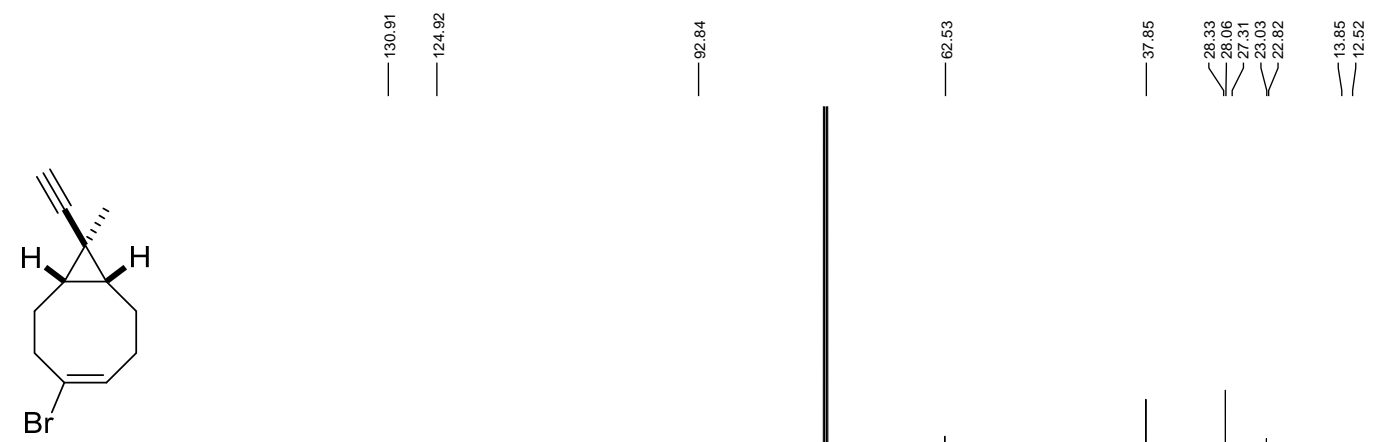

13

$125 \mathrm{MHz}, \mathrm{CDCl}_{3}$

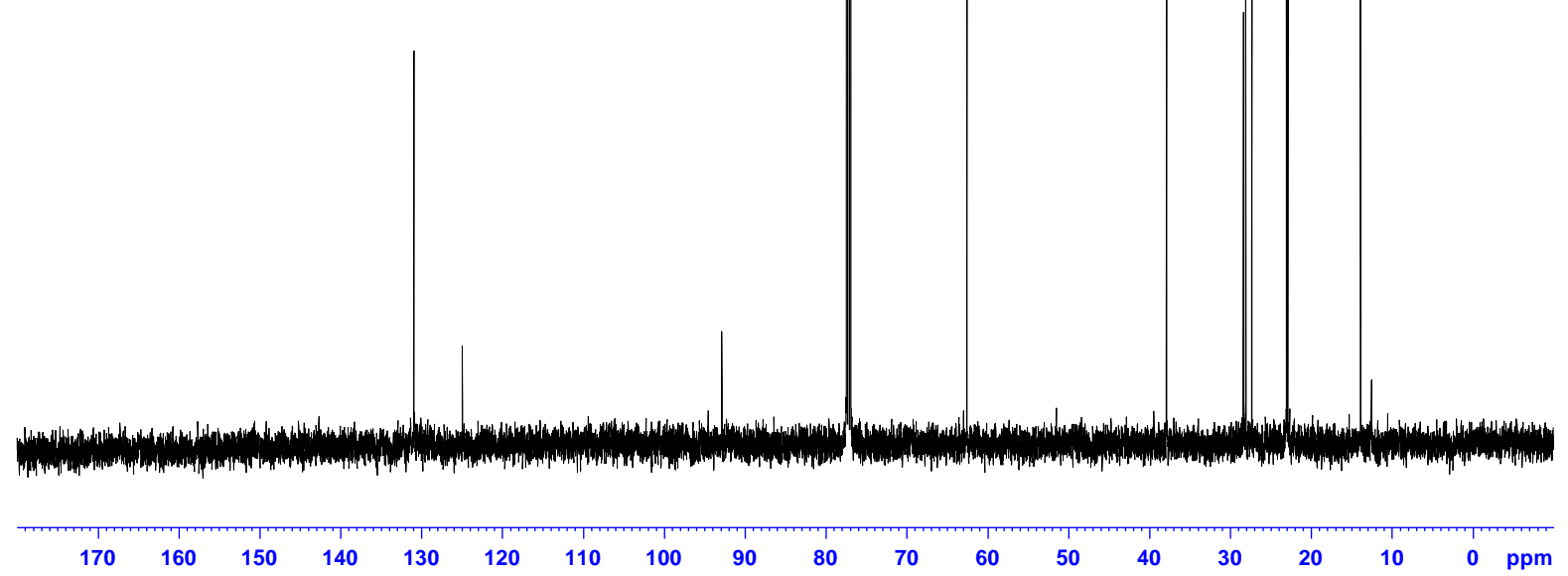




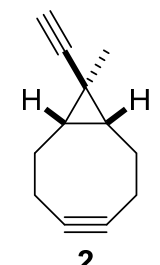

m

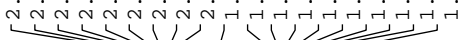

$300 \mathrm{MHz}, \mathrm{CDCl}_{3}$
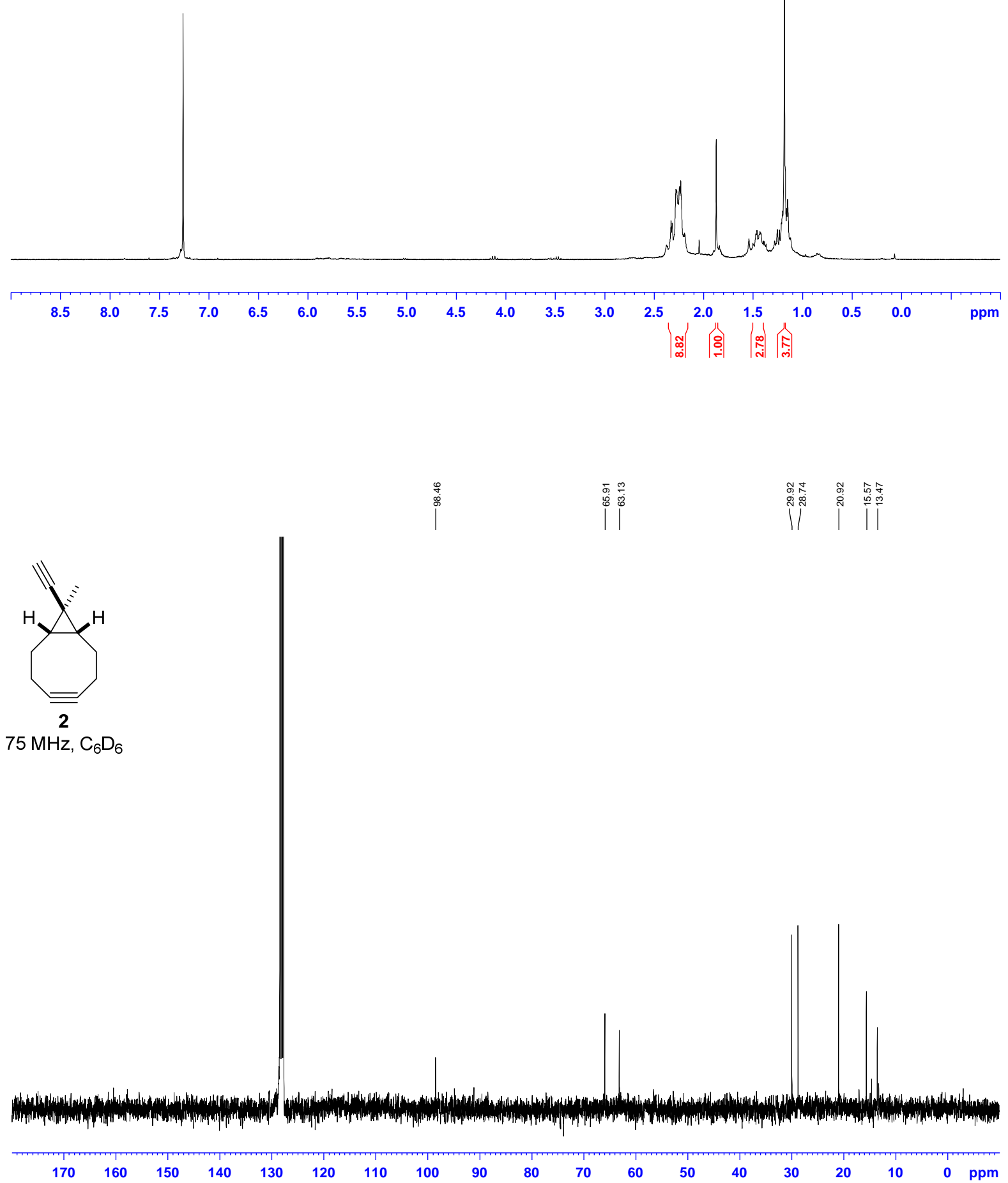


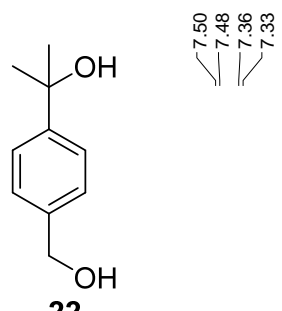

$\stackrel{\substack{\infty \\+}}{+\infty}$

$300 \mathrm{MHz}, \mathrm{CDCl}_{3}$
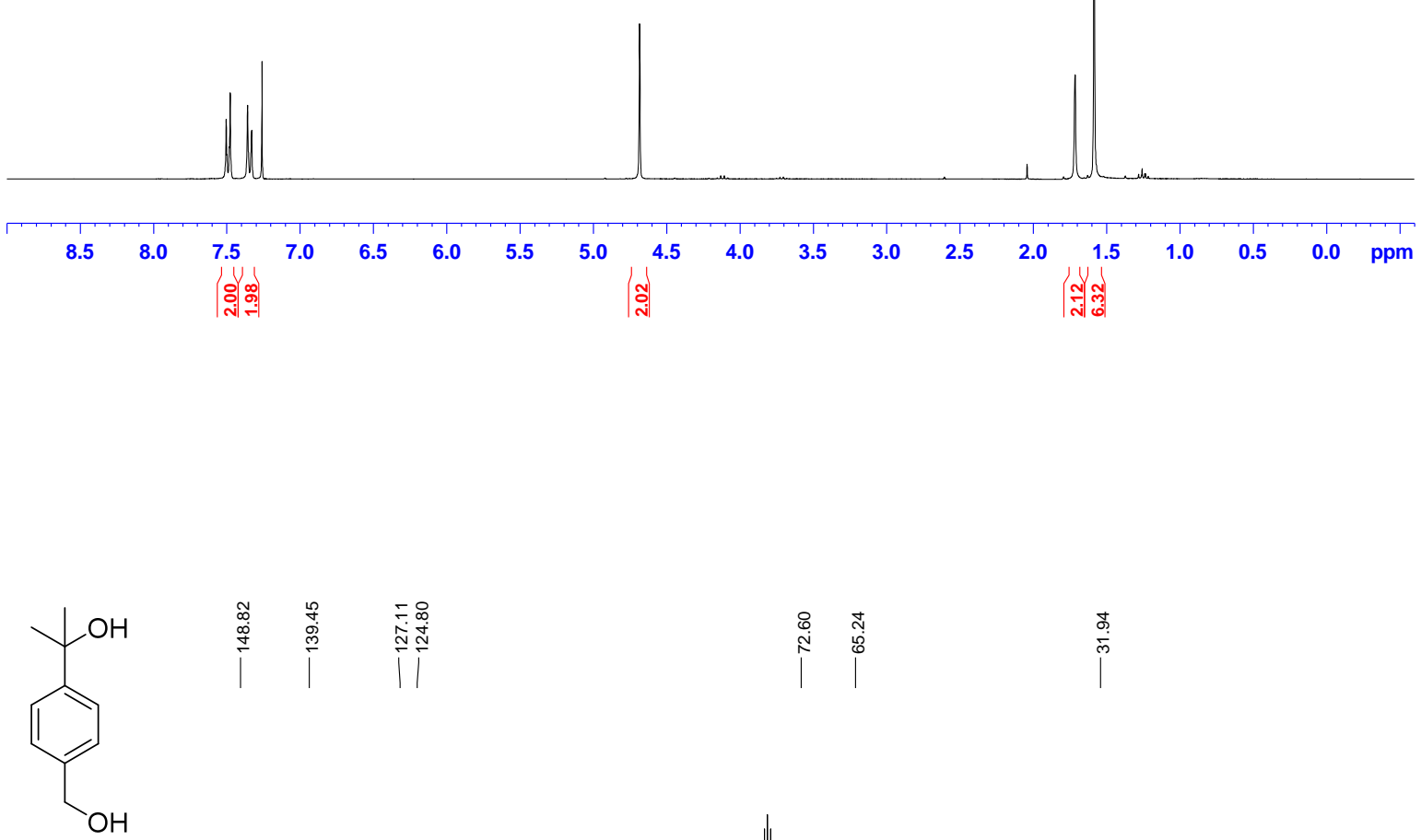

|

22

$75 \mathrm{MHz}, \mathrm{CDCl}_{3}$

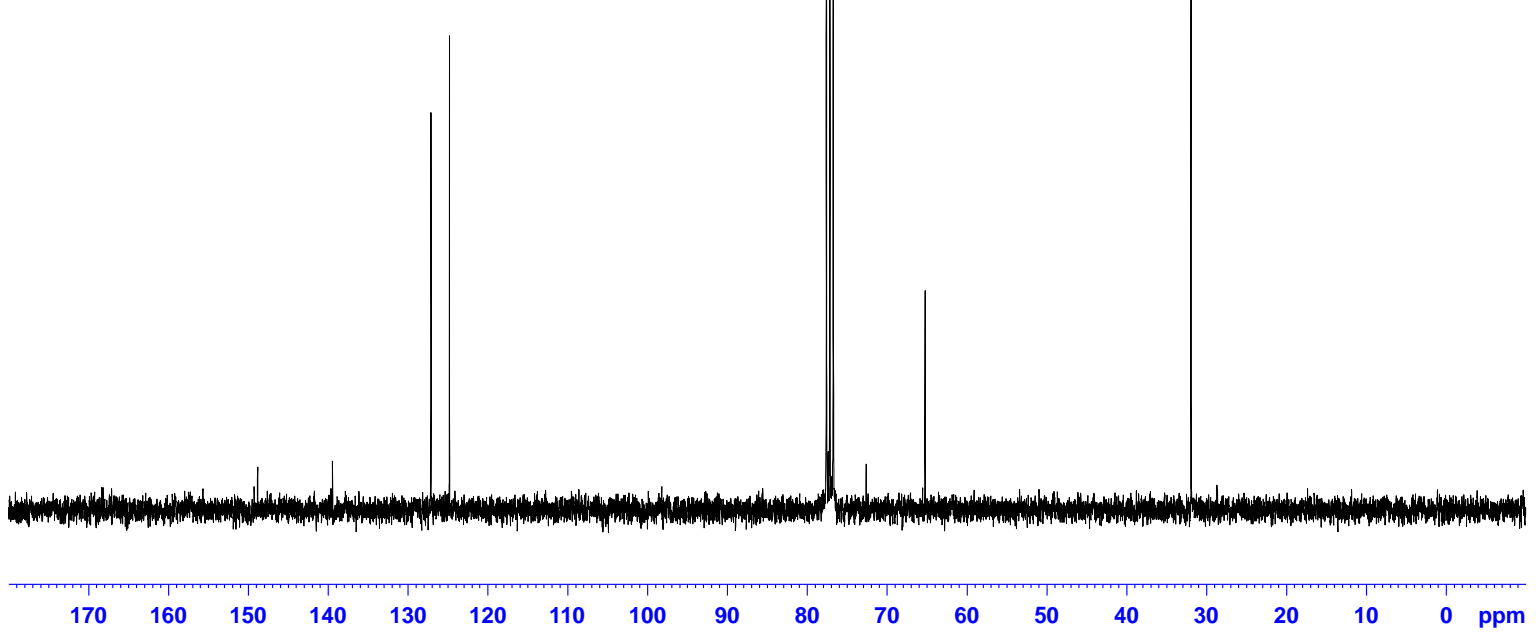




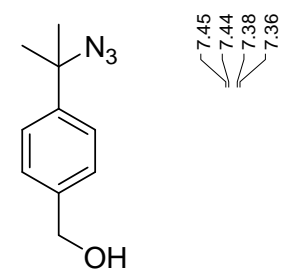

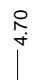

$500 \mathrm{MHz} \mathrm{CDCl}_{3}$
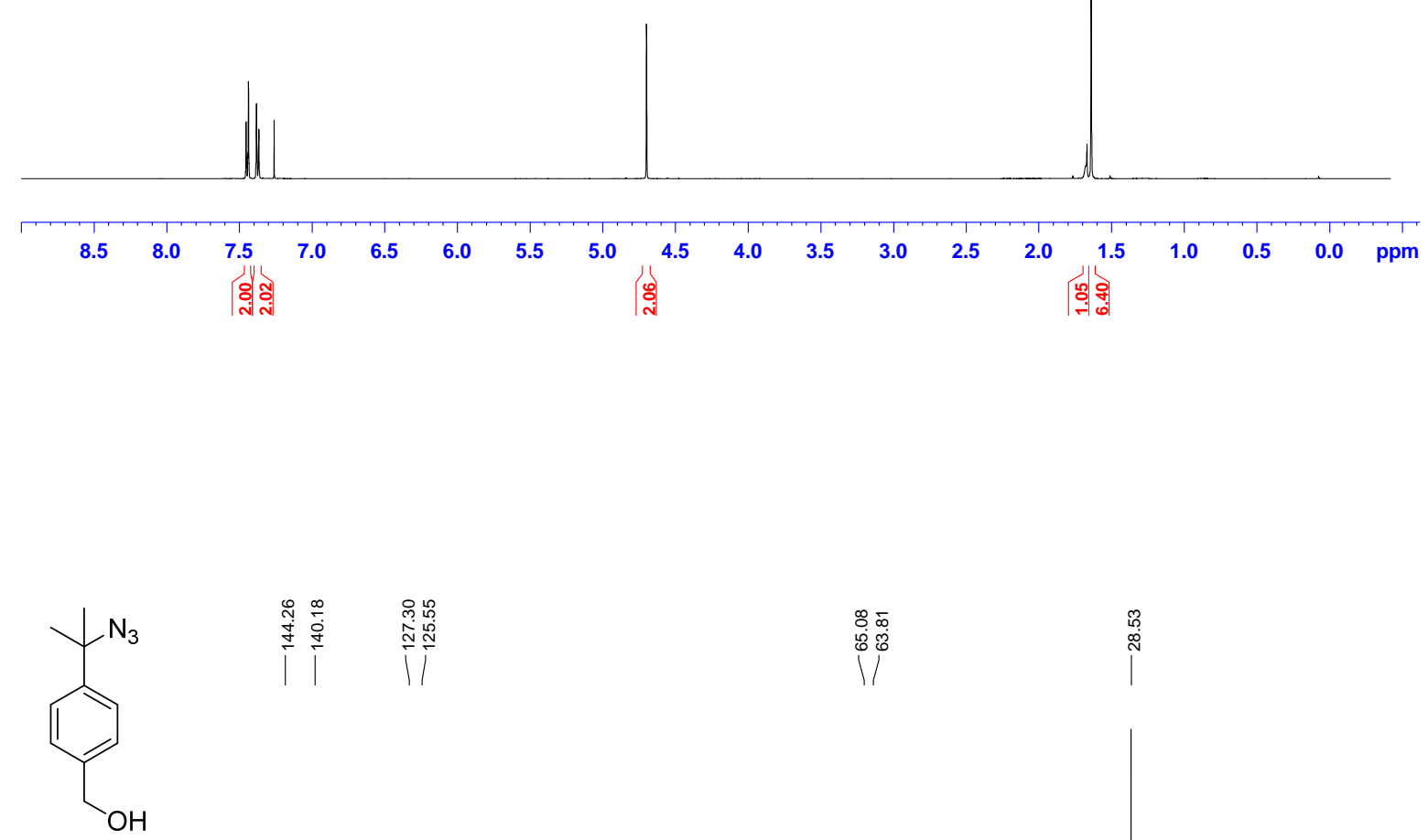

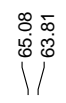

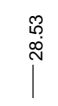

$125 \mathrm{MHz}, \mathrm{CDCl}_{3}$

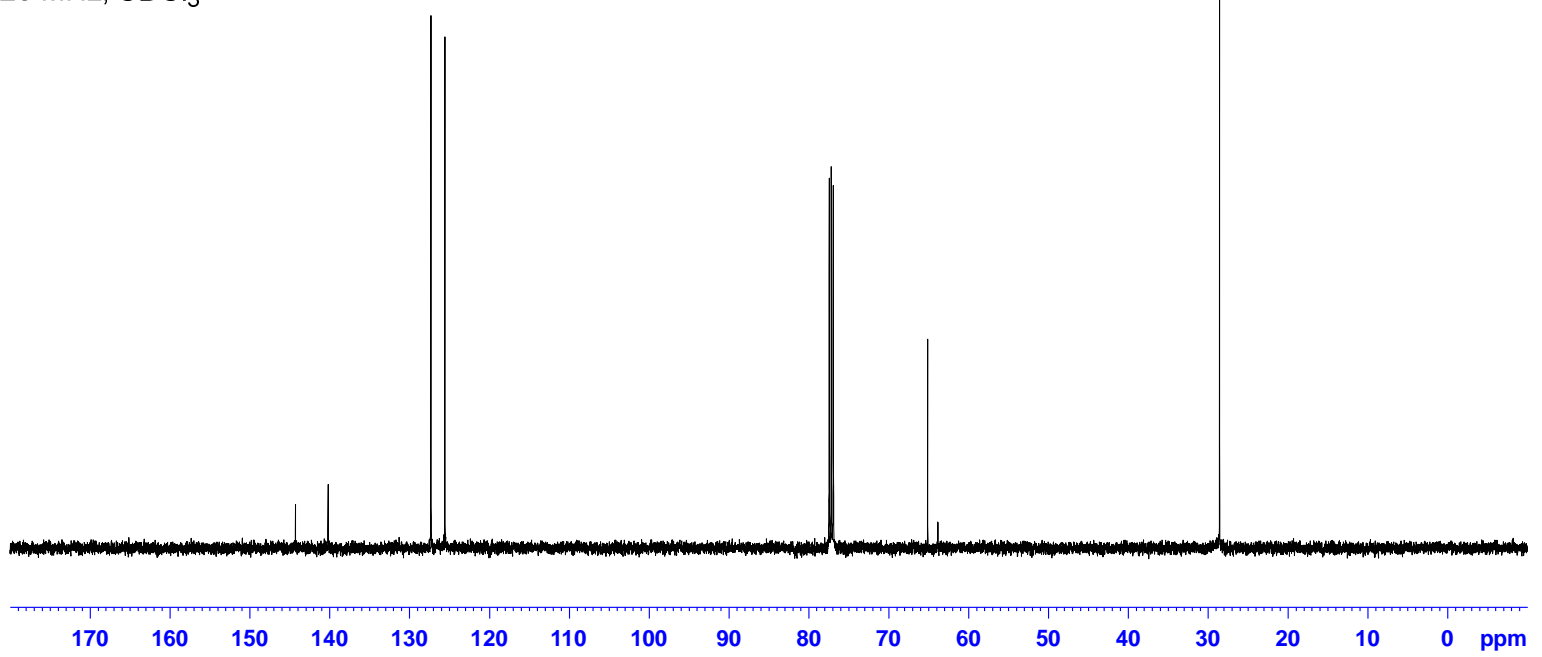



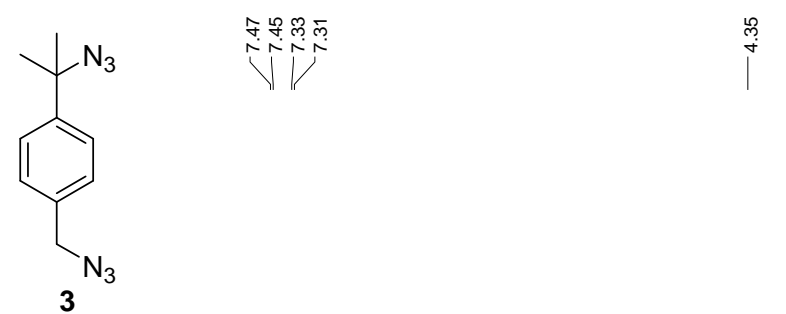

$500 \mathrm{MHz}, \mathrm{CDCl}_{3}$
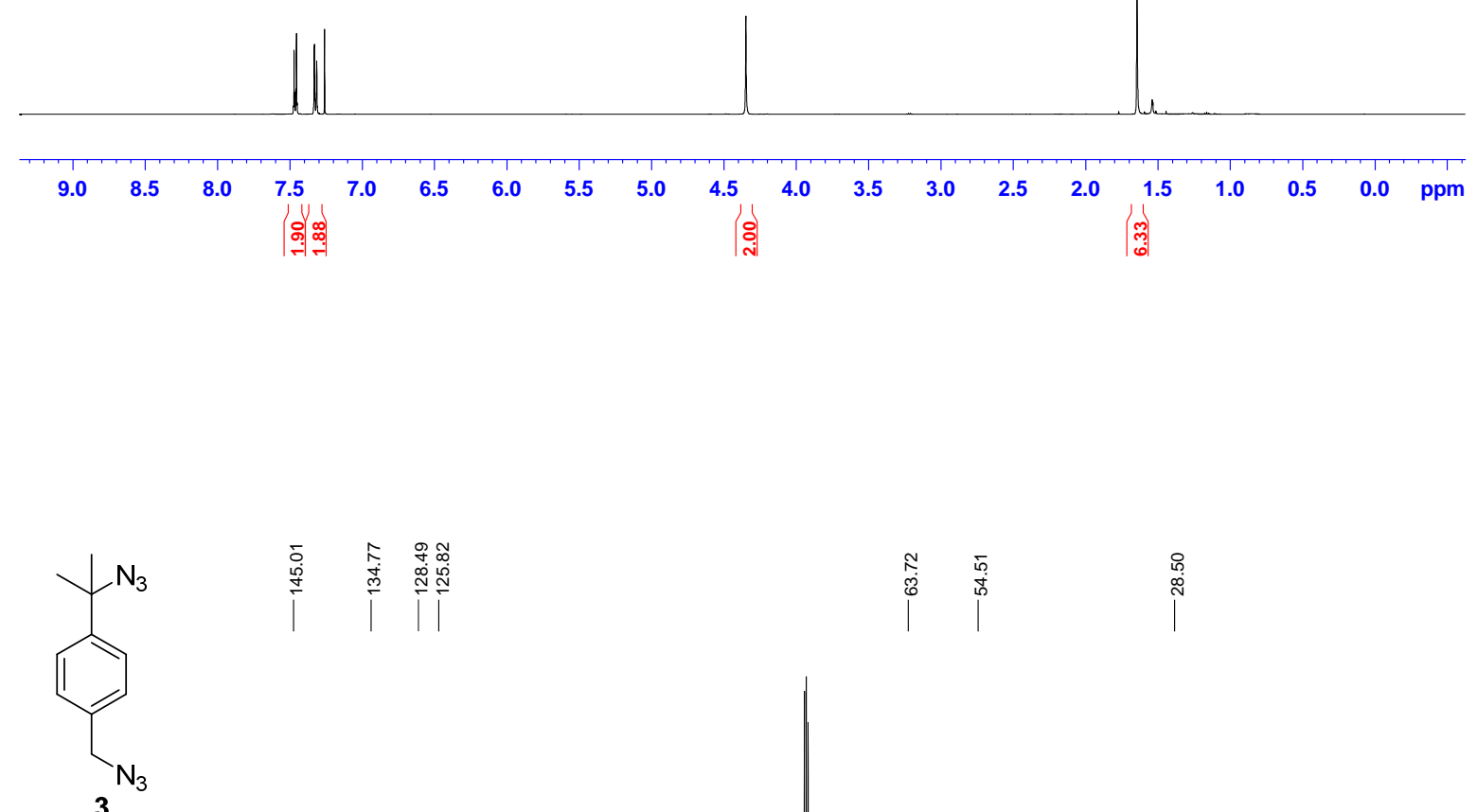

$125 \mathrm{MHz}, \mathrm{CDCl}_{3}$

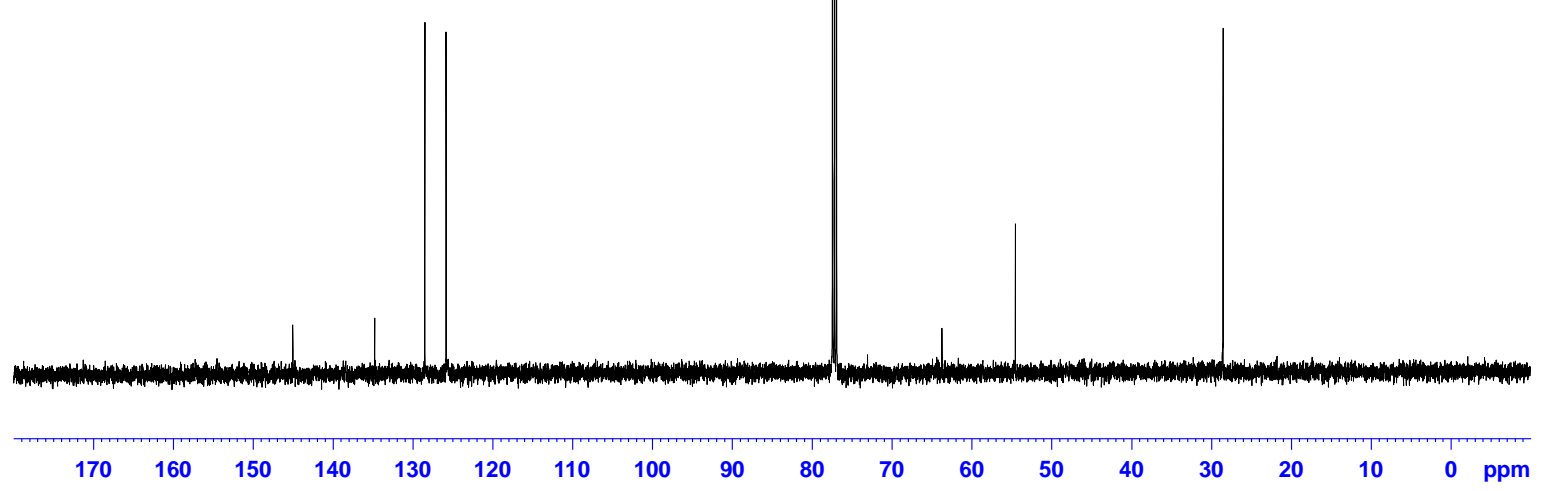




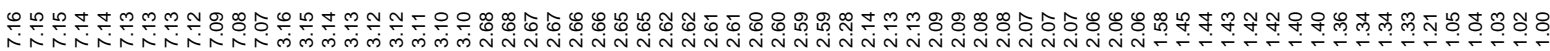

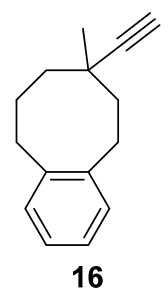

$600 \mathrm{MHz}, \mathrm{CDCl}_{3}$
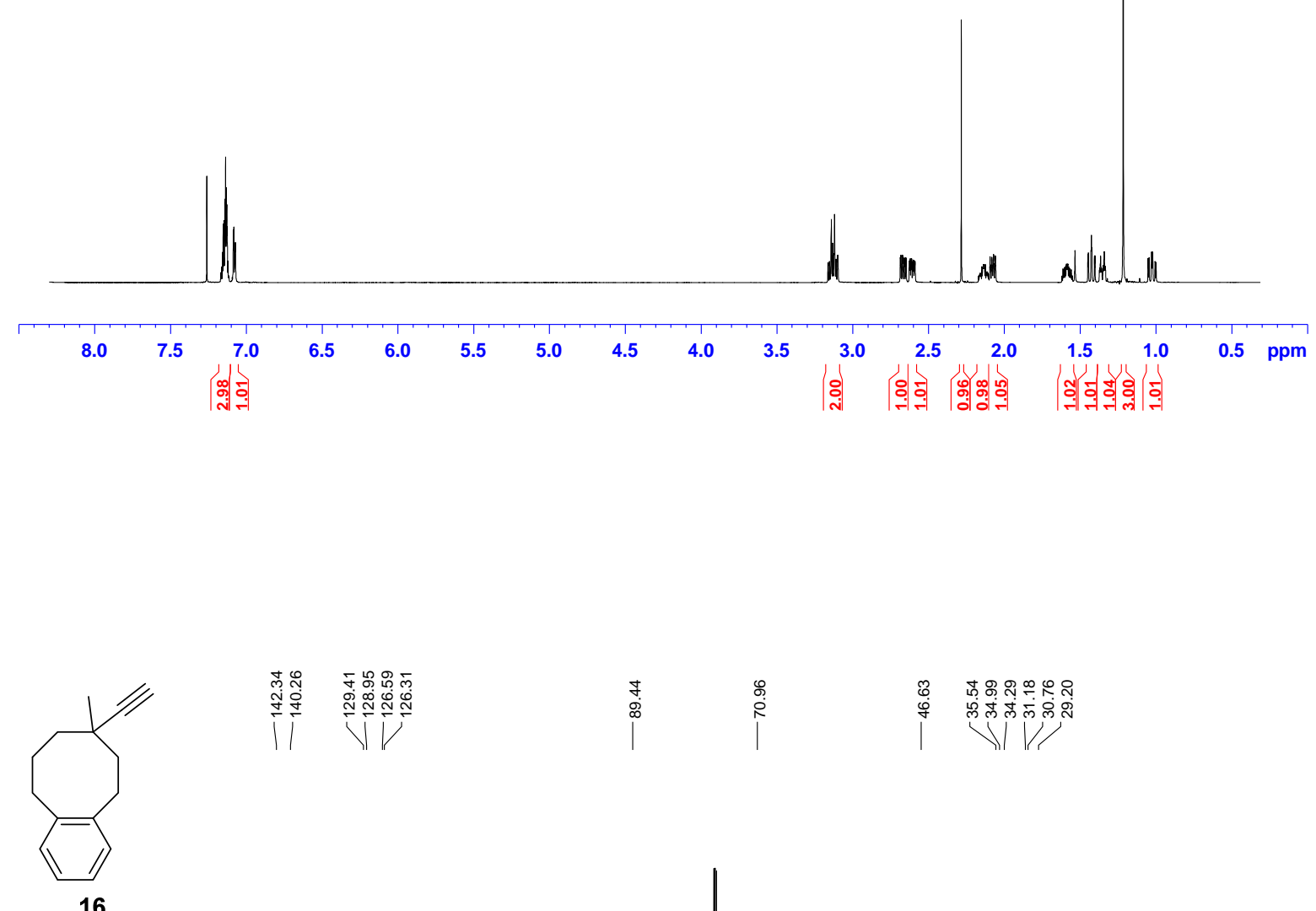

$151 \mathrm{MHz}, \mathrm{CDCl}_{3}$

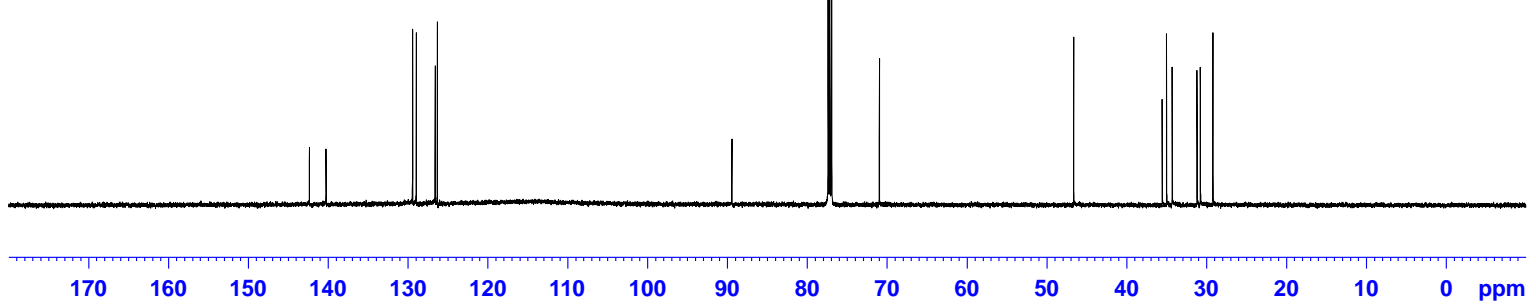




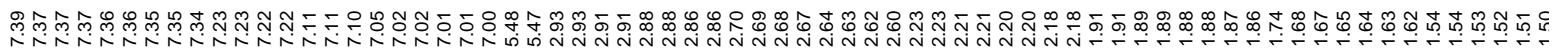

$=$

19

$500 \mathrm{MHz}, \mathrm{CDCl}_{3}$
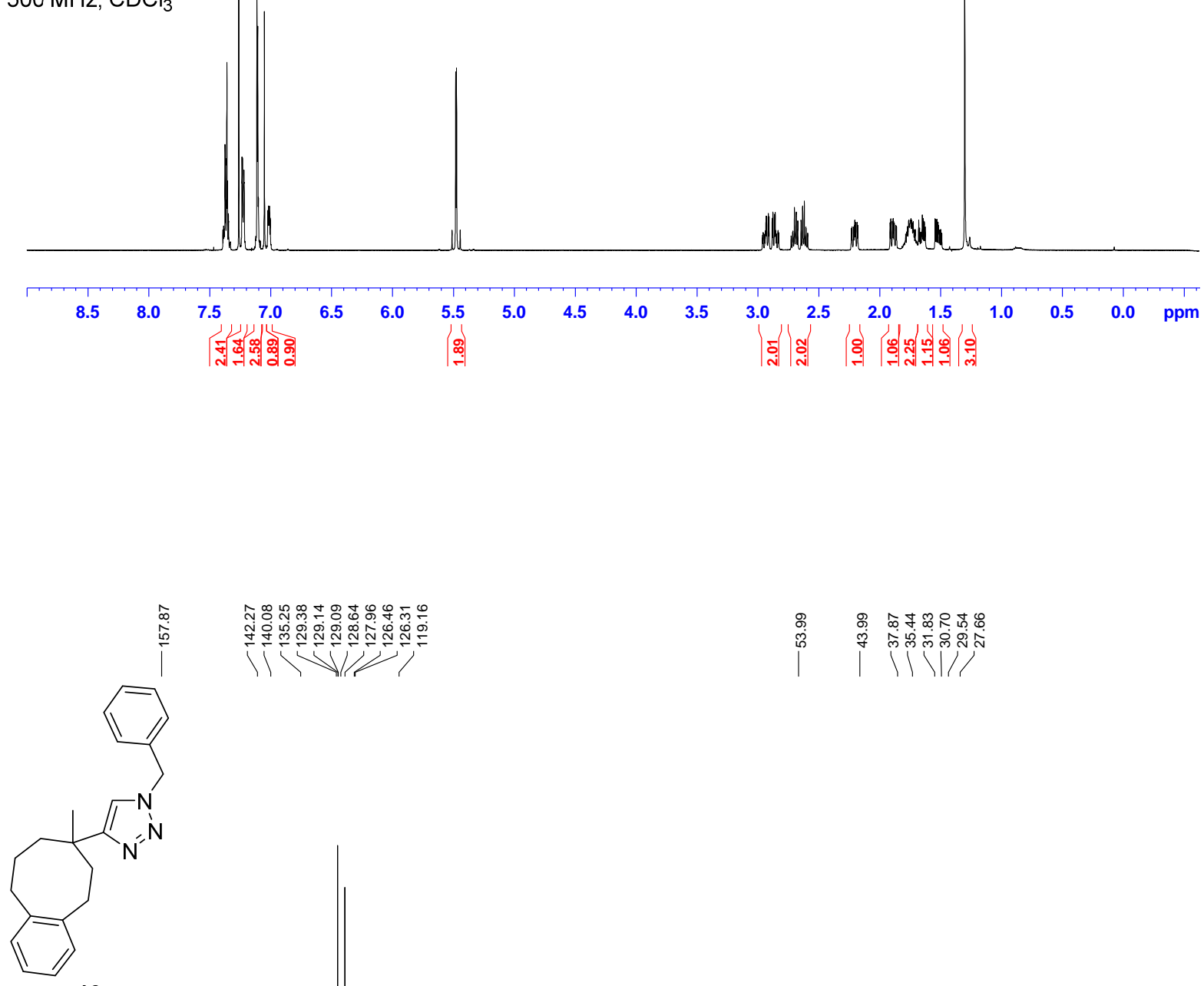

19

$125 \mathrm{MHz}, \mathrm{CDCl}_{3}$

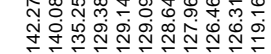

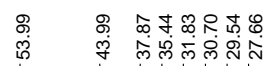

1 1 1111

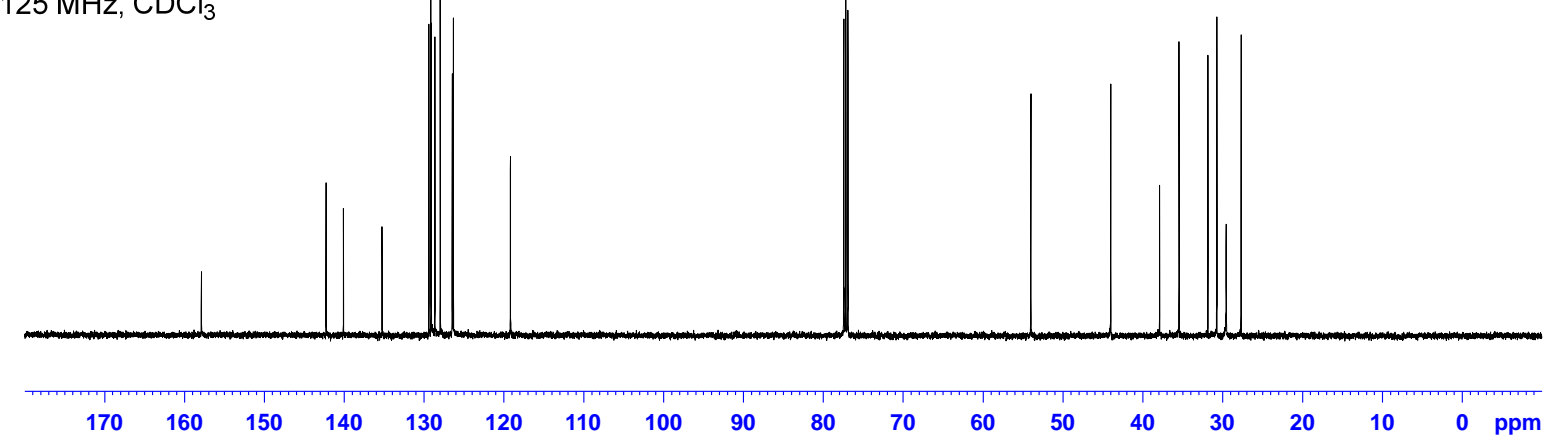




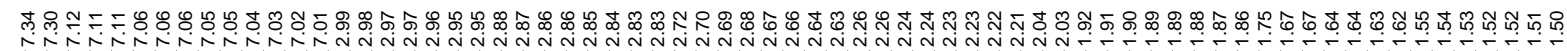

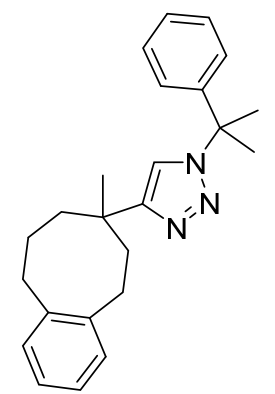

20

$500 \mathrm{MHz}, \mathrm{CDCl}_{3}$

Wh Whan
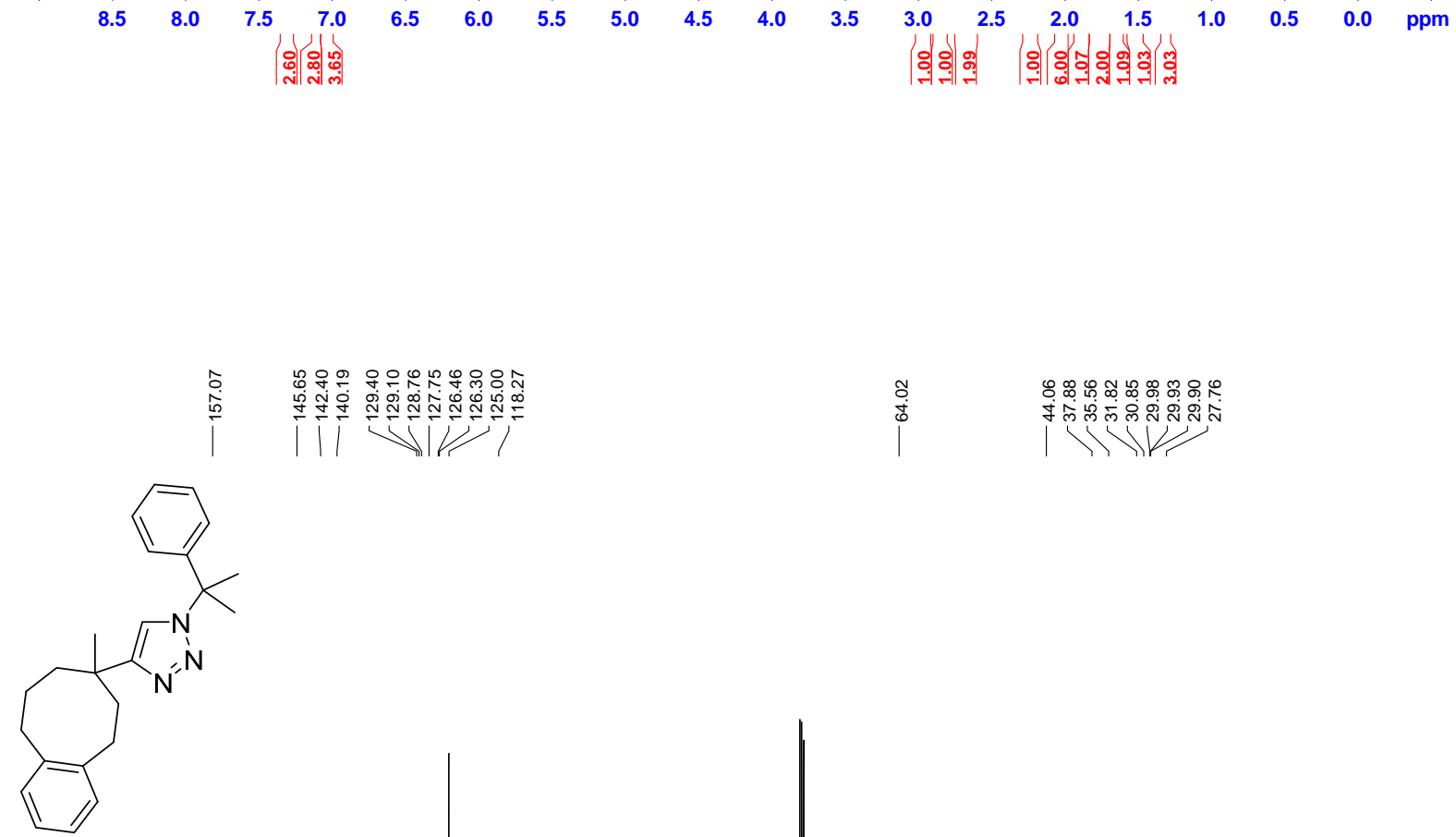

20

$125 \mathrm{MHz}, \mathrm{CDCl}_{3}$

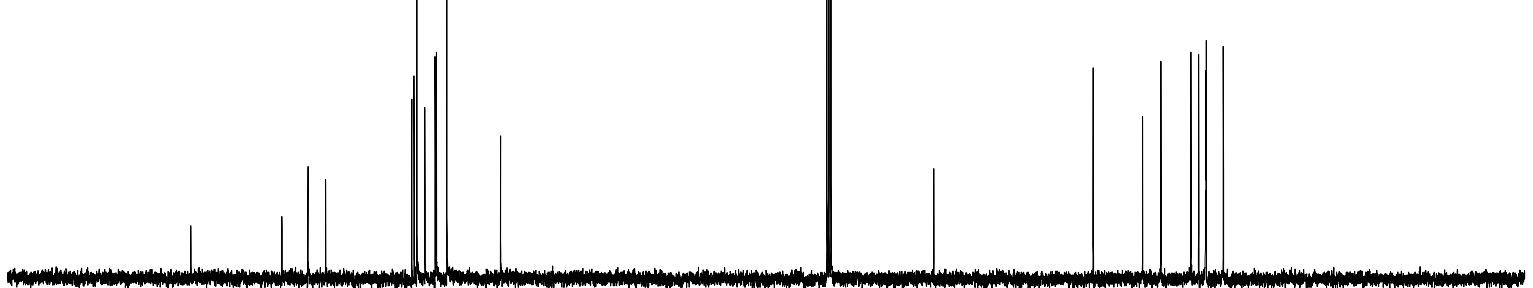

$\begin{array}{lllllllllllllllllll}170 & 160 & 150 & 140 & 130 & 120 & 110 & 100 & 90 & 80 & 70 & 60 & 50 & 40 & 30 & 20 & 10 & 0 & \mathrm{ppm}\end{array}$ 


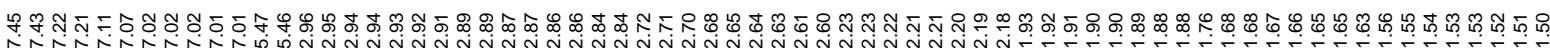

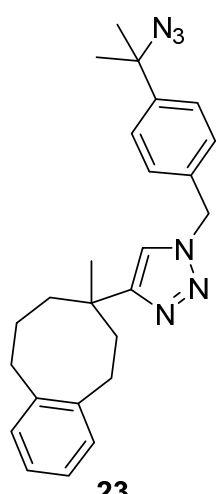

$500 \mathrm{MHz}, \mathrm{CDCl}_{3}$

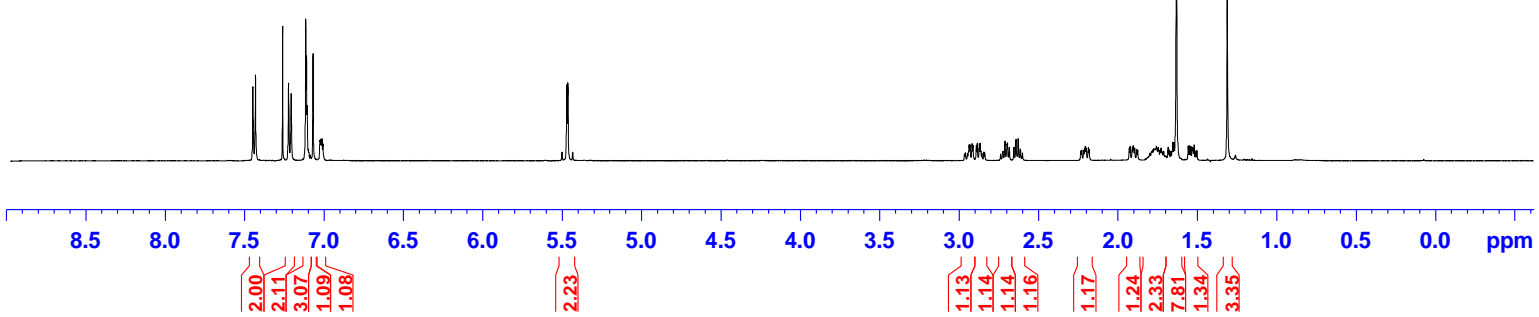

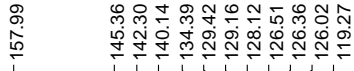

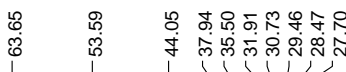

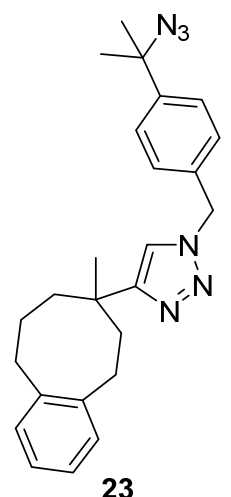

23

$125 \mathrm{MHz}, \mathrm{CDCl}_{3}$

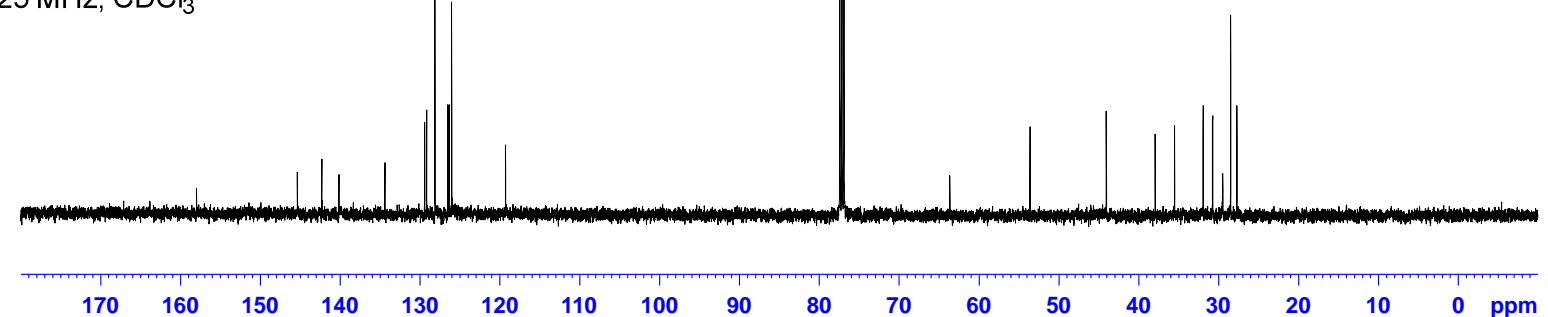



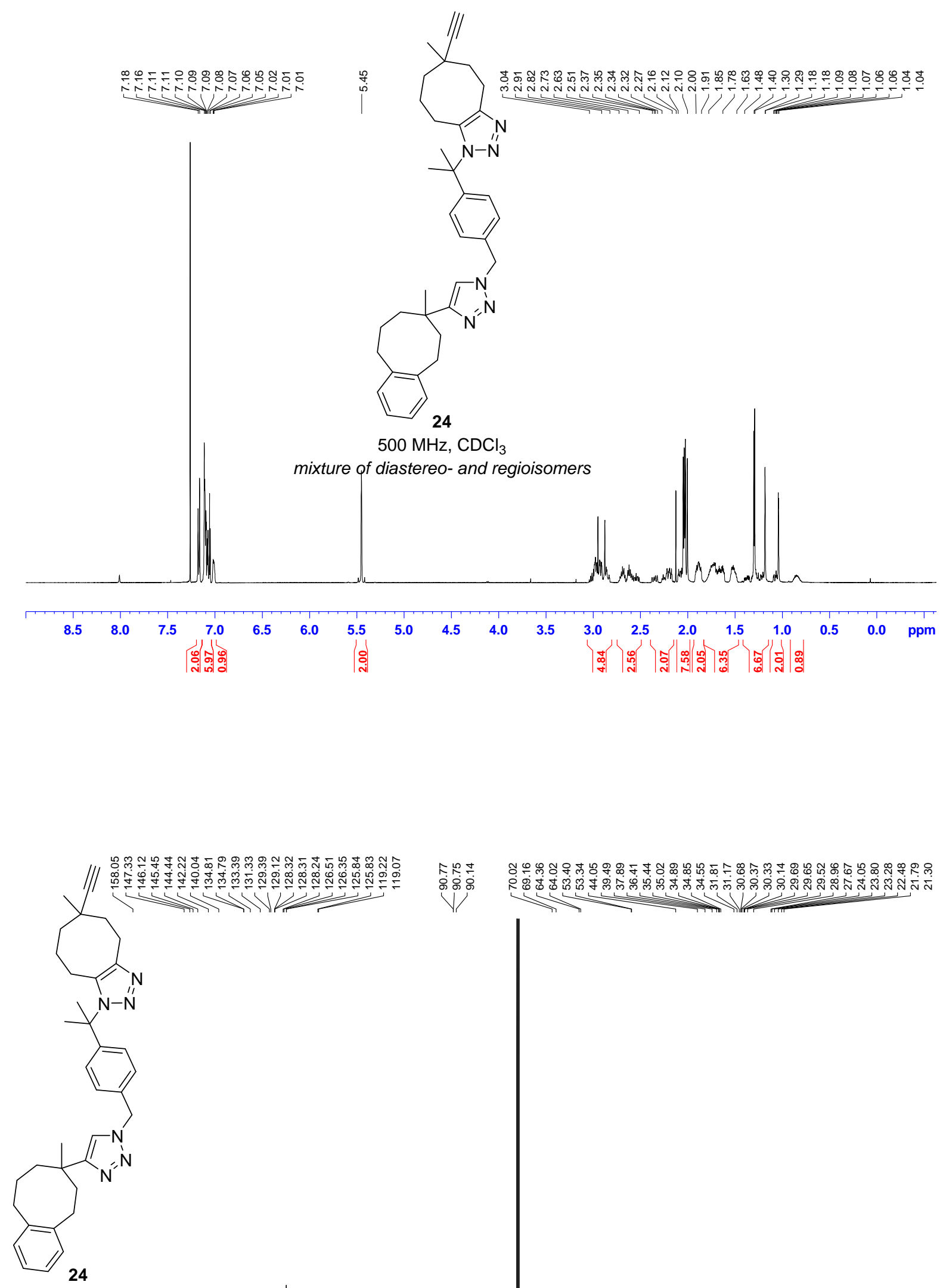

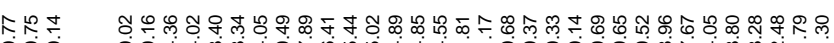

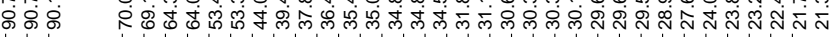
V)

$125 \mathrm{MHz}, \mathrm{CDCl}_{3}$

mixture of diastereo- and regioisomers

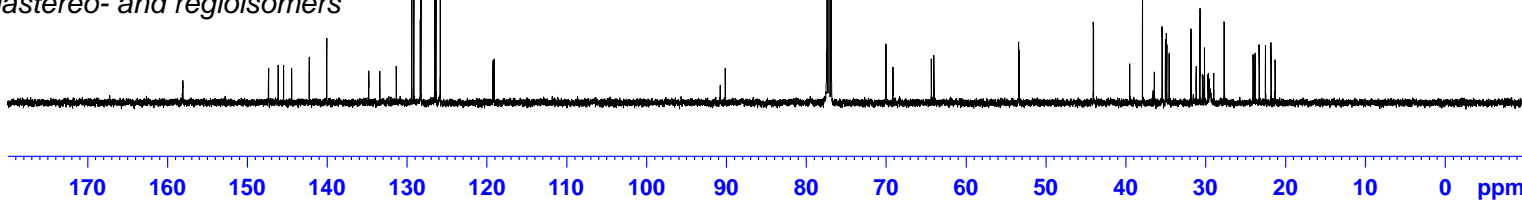




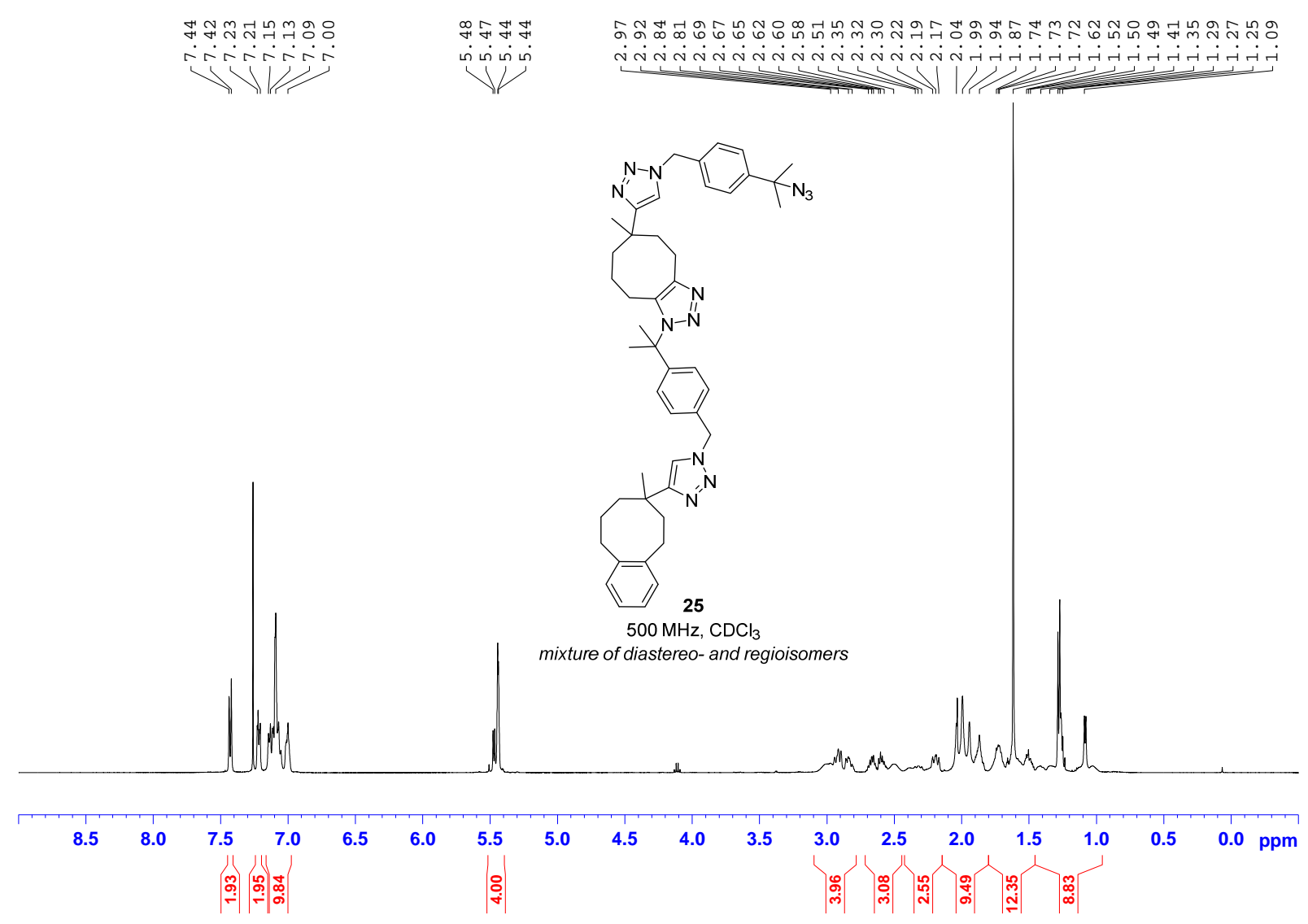

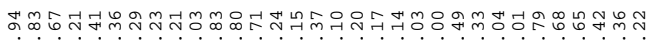

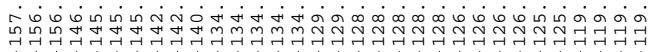
$\underbrace{-10}$

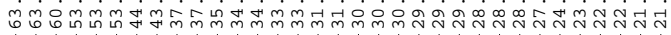

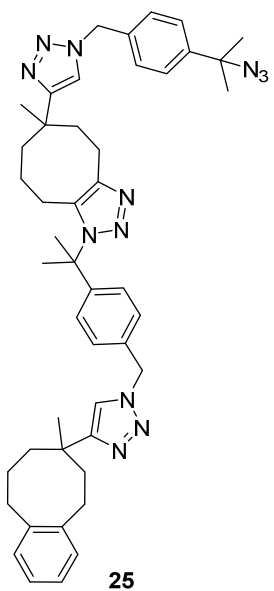

$125 \mathrm{MHz}, \mathrm{CDCl}_{3}$ mixture of diastereo- and regioisomers

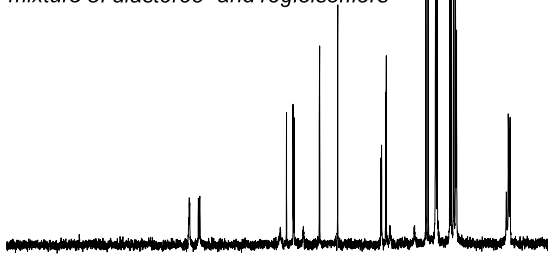

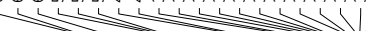

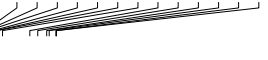

$\begin{array}{llllllll}170 & 160 & 150 & 140 & 130 & 120 & 110 & 100\end{array}$

60

50

40

30 

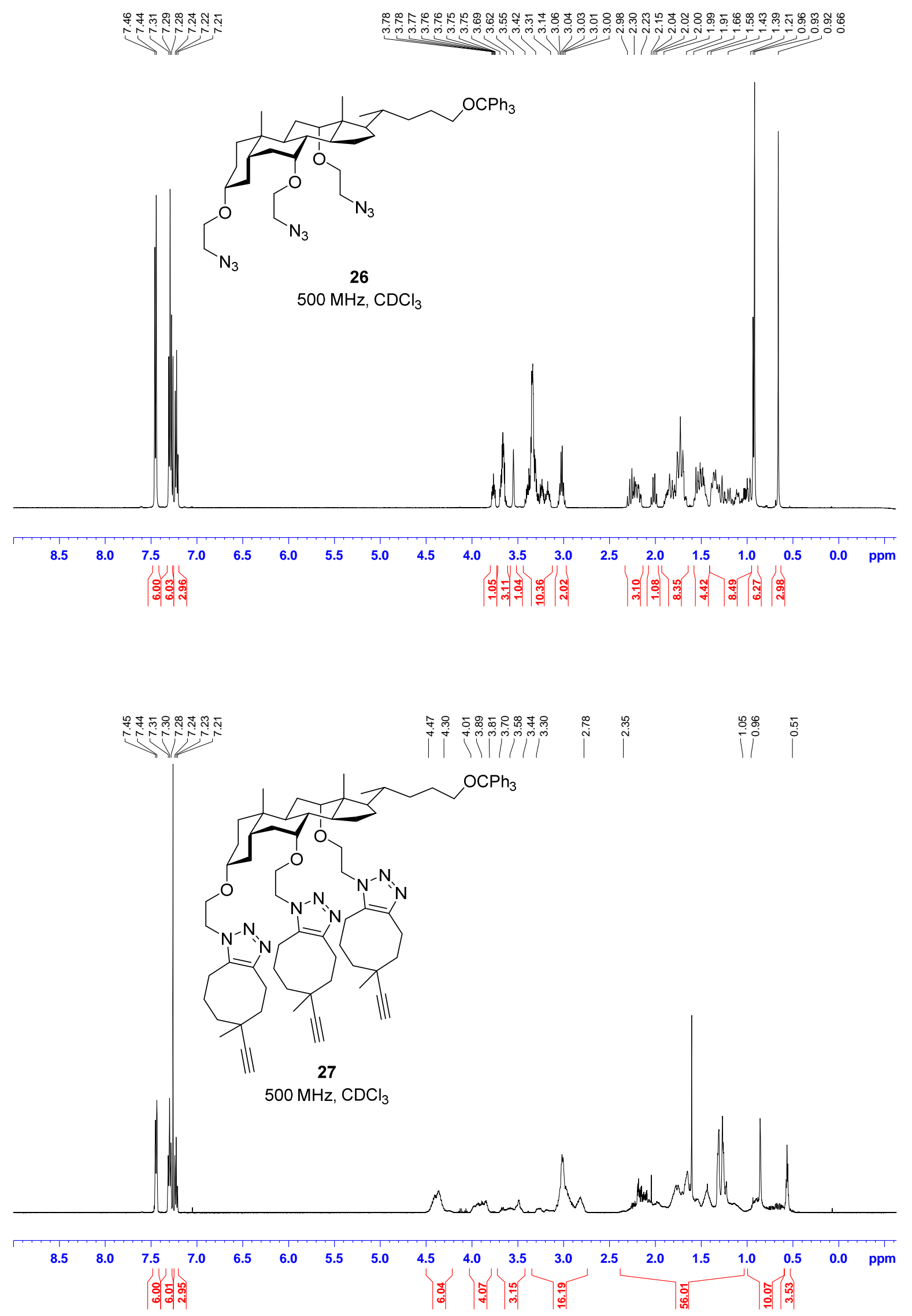

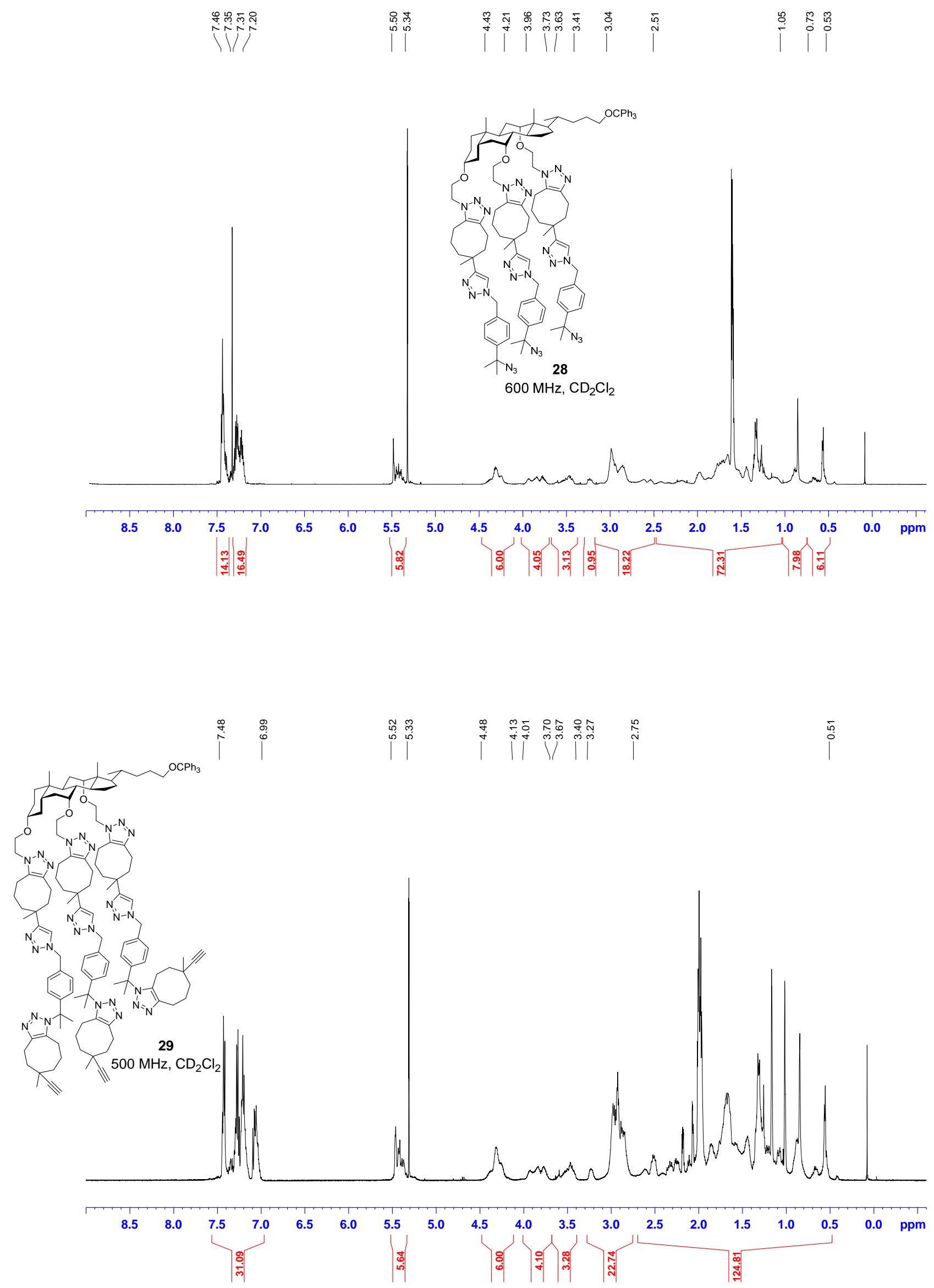

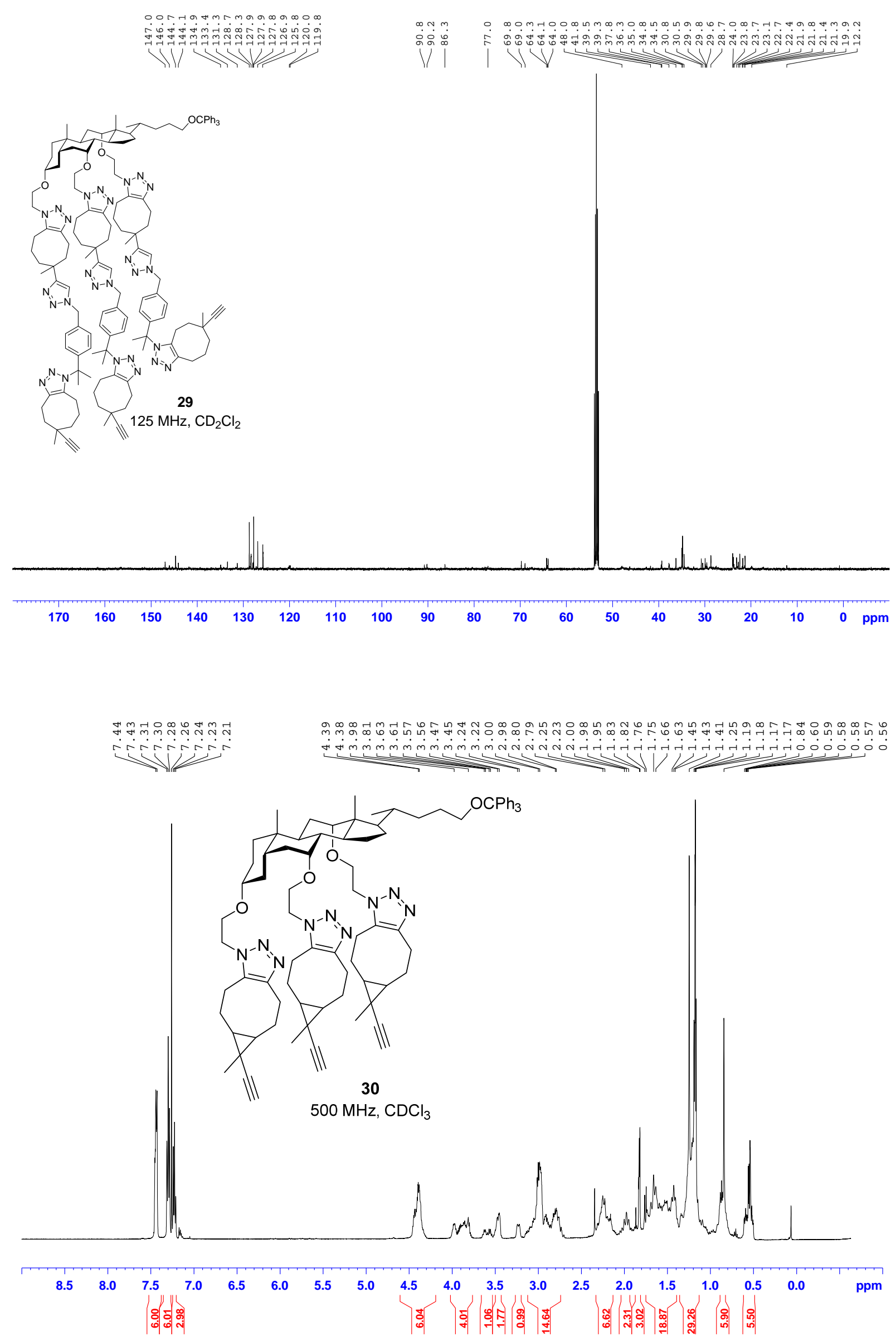

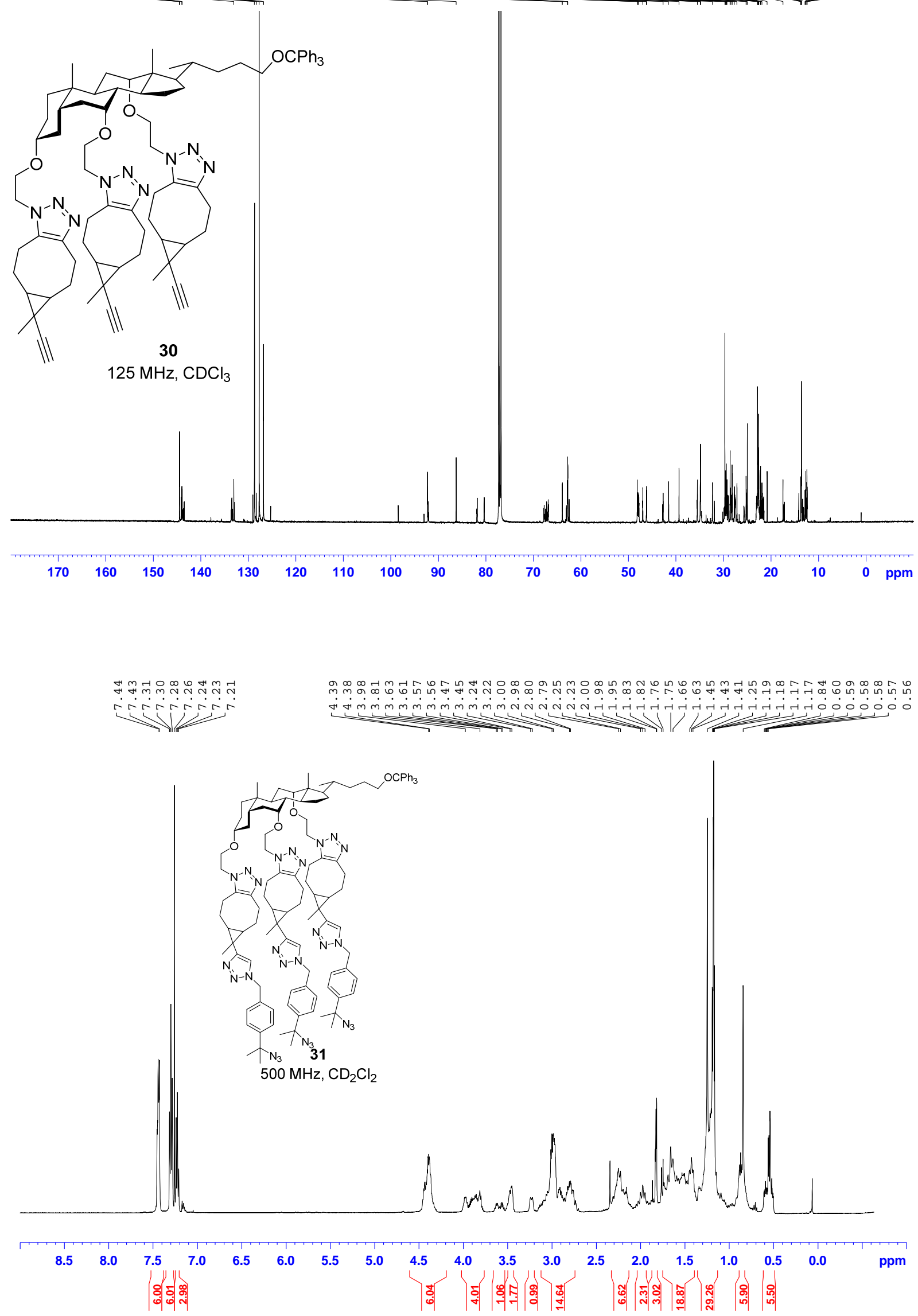

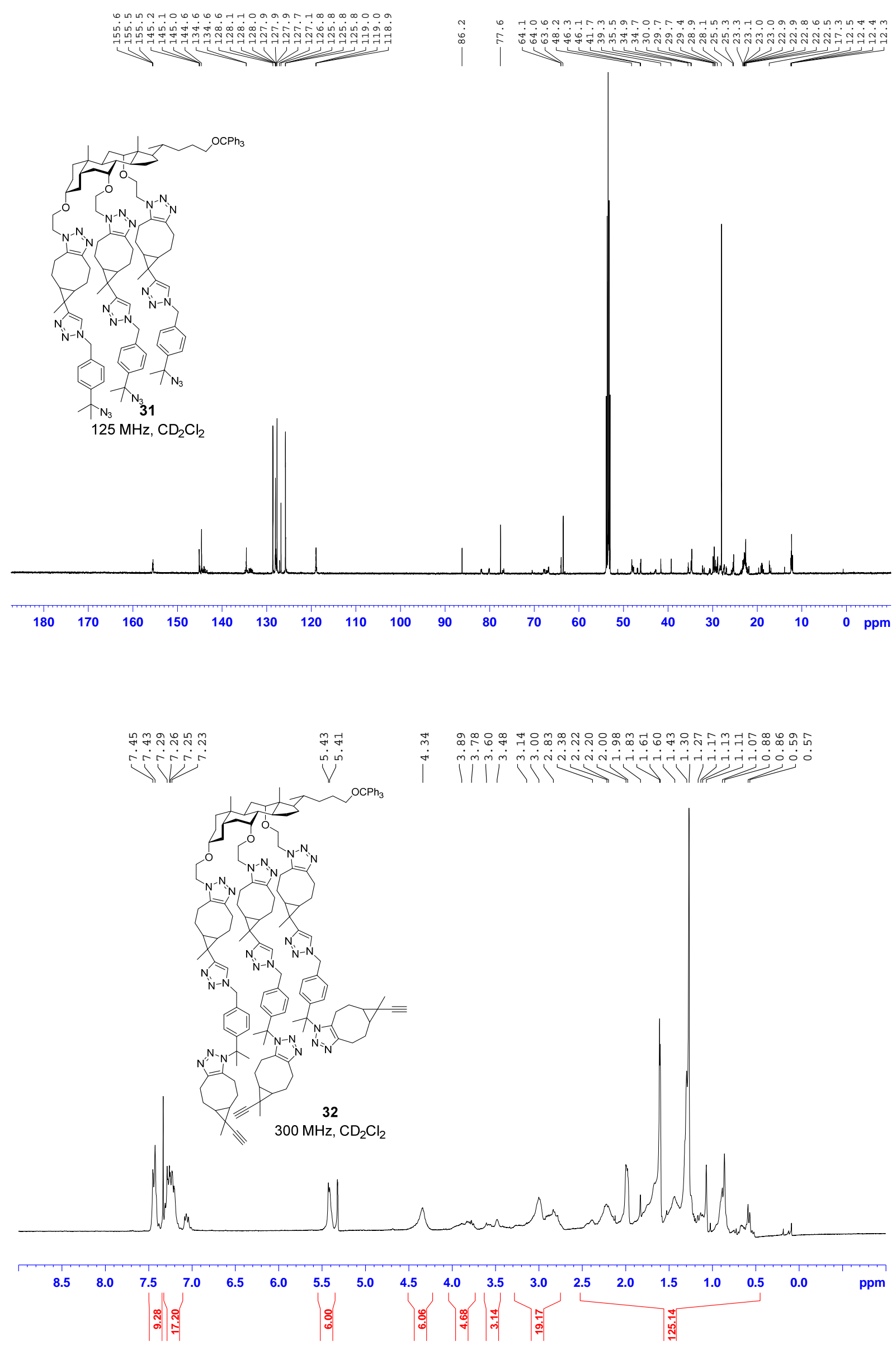


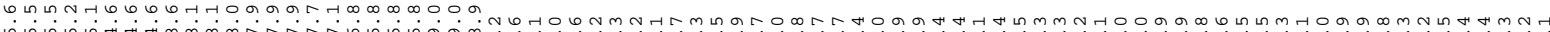

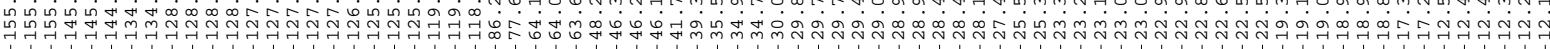

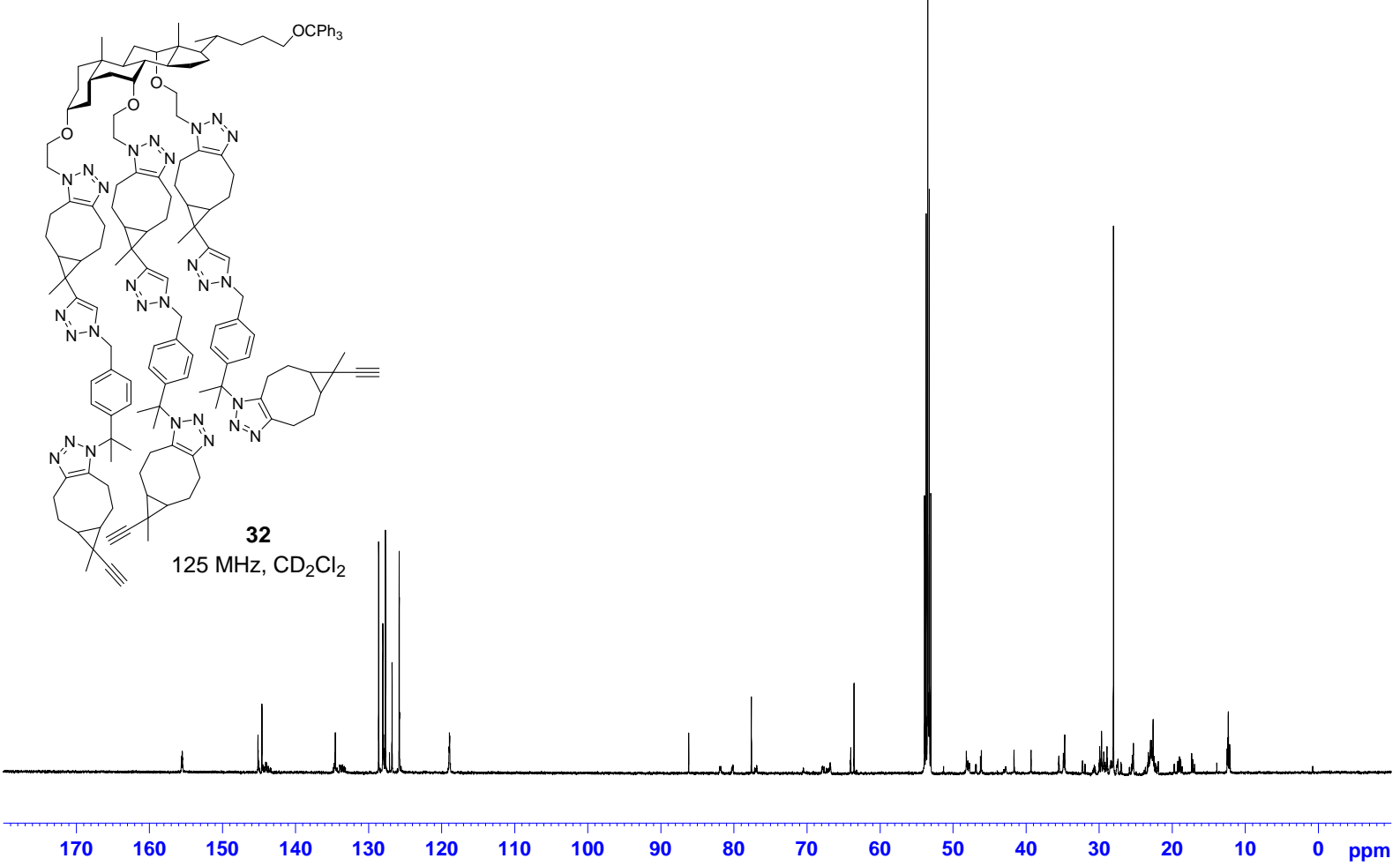

